\title{
Germanium Blocked Impurity Band Far Infrared Detectors
}

\author{
Carolyn Sally Rossington \\ (Ph.D. Thesis) \\ Lawrence Berkeley Laboratory \\ University of Califomia \\ Berkeley, California 94720
}

April 1988

\section{DISCLAIMER}

This report was prepared as an account of work sponsored by an agency of the United States Government. Neither the United States Government nor any agency thercof, nor any of their employees, makes any warranty, express or implied, or assumes any legal liability or responsibility for the accuracy, cumpleteness, or usefulness of any information, apparatus, product, or process disclased, or represents that its use would not infringe privately owned rights. Reference herein to any specific commercial product, process, or service by trade name, trademark, manufacturer, or otherwise docs not necessarily constitute or imply its endorsement, recommendation, or favoring by the United States Government or any agency thereof. The views and opinions of authors expressed herein do not necessarily state or reflect those of the United States Government or any agency thercof. 


\section{ACKNOWLEDGEMENTS}

I would like to thank Professor Eugene Haller for taking me on as a graduate student in the middle of my graduate studies at Berkeley and for the continuous support he gave me during the course of this research project. Many other people in our group here at. Lawrence Berkeley Lab also contributed to this work, and special thanks go to Bill Hansen, Dick Davis, Jeff Beeman, Bob McMurray, In-shik Park, Kin-man $Y u$ and In-chin Wu. Thanks go to NASA for two years of personal support in the form of a Graduate Student Researchers Fellawship, and for two years of scientific support in the form of NASA Contract W-14606, under Interagency Agreement with the Director's Office of Energy Research, Office of Health and Environmental Research, U.S. Department of Energy under Contract No. DE-ACO3-76SF00098. 
TABLE OF CONTENTS

1.0 Introduction.......................................

1.1 Infrared and Infrared Detection.......................

1.2 Blocked Impurity Band Detectors......................

1.2.1 8IB Detector Physics..........................

1.2.2 Review of Silicon and Germanium BIB Detectors...........17

2.0 Semiconductor Epitaxy..............................23

2.1 Introduction to Semiconductor Epitaxy.................23

2.2 Germanium Epitaxy.............................27

2.2.1 Epitaxy using the Vertical CVD Chamber...............27

2.2.1.1 Apparatus and Epitaxy Procedure...............27

2.2.1.2 Electrical Characterization of the Epilayers........36

2.2.1.3 Structural Characterization of the Epilayers.......42

2.2.1.4 Sumary of Epitaxy using the Vertical CVD Chamber....50

2.2.2 Epitaxy using the Horizontal CVD Chamber.............51

2.2.2.1 Apparatus and Epitaxy Procedure................51

2.2.2.2 Electrical Characterization of the Epilayers........60

2.2.2.3 Structural Characterization of the Epilayers........65

2.2.2.4 Sumary of Epitaxy using the Horizontal Chamber......73

3.0 Germanium BIB Detectors.............................. 75

3.1 BIB Detector Fabrication........................ 75

3.2 Pertinent Detector Parameters..........................

3.3 Detector Test Apparatus and Procedure.................81

3.4 Detector Results and Discussion...................... 84

3.4 .1 Results from the $5 \times 10^{15} \mathrm{~cm}^{-3} \mathrm{Ge}: \mathrm{Ga}$ BIB Detector.....84 


\section{TABLE OF CONTENTS}

3.4.1.1 Dark Current..............................85

3.4.1.2 Responsivity.............................92

3.4.1.3 Noise Equivalent Power......................99

3.4.2 Results from the $2 \times 10^{16} \mathrm{~cm}^{-3} \mathrm{Ge}: \mathrm{Ga}$ BIB Detector......99

3.4.2.1 Dark Current..............................101

3.4.2.2 Responsivity,.............................

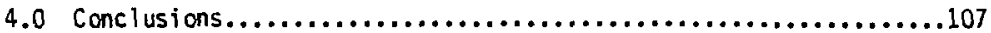

References............................................110

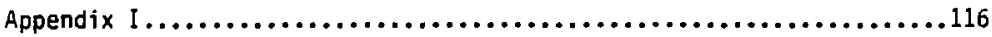

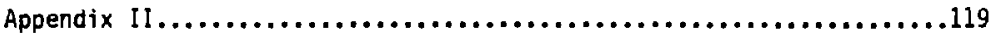

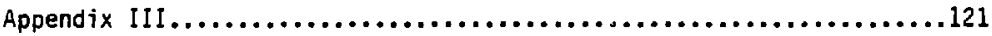

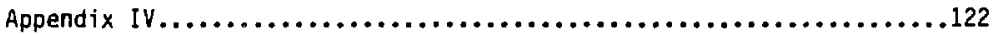




\subsection{Introduction}

\subsection{Infrared and Infrared Detection}

The infrared portion of the electromagnetic spectrum has been of interest to scientists since the eighteenth century when Sir William Herschel discovered the infrared as he measured temperatures in the sun's spectrum and found that there was energy beyond the red (1). In the late nineteenth century, Thomas Edison established himself as the first infrared astronomer to look beyond the solar system when he observed the star Arcturus in the infrared. Significant advances in infrared technology and nhysics, long since Edison's time, have resulted in many scientific developments, such as the Infrared Astronomy Satellite (IRAS) which was launched in 1983 (2), semiconductor infrared detectors for materials characterization, military equipment such as night-vision goggles and infrared surveillance equipment. It is now planned that cooled semiconductor infrared detectors will play a major role in the "Star Wars" nuclear defense scheme proposed by the Reagan administration. Extrinsic germanium infrared detectors, a class of which was studied in this work, are of particular use in civilian scientific applications. For example, astronomers and astrophysicists have a strong interest in the infrared range between -15 and $250 \mu \mathrm{m}$, where these detectors respond. Such detectors, among others, were utilized on IRAS and contributed to the detection of over 250,000 previously unknown infrared sources in our galaxy and beyond (2). Radiation from galactic sources in the infrared reveals information about the molecular makeup of these sources, as the majority of strong molecular spectral lines lies in the infrared. The 
successes of IRAS have led to the development of the Space Infrared Telescope Facility (SIRTF), a much more challenging followup mission, by the National Aeronautics and Space Administration (NASA). SIRTF will carry out photometry over the wavelength range 1.8-700 $\mathrm{mm}$ and is designed to be at least 1000 times more sensitive than IRAS, due to the advances in infrared detectors and instrumentation which have occurred since IRAS (3). It is hoped that SIRTF, which is scheduled for launch in the mid 1990's, will provide the key to understanding cosmic birth -- the formation of planets, stars, galaxies and quasars.

\subsection{Blocked Impurity Band Detectors}

Germanium Blocked Impurity Band (BIB) detectors fall under the broad category of extrinsic semiconductor photoconductors. To begin a discussion of BIB detectors, it is helpful to briefly review photoconductors and photoconductivity. A photoconductor is a device in which a current is generated as a consequence of an absorbed incident photon flux. The photon energy to which the photoconductor responds is determined by the type of material and the physical characteristics of the device. Intrinsic semiconductors, such as GaAs, Ge and Si can act as photoconductors, in addition to such ternary compounds as HgCdTe and PbGeTe (4). The photon energy to which the intrinsic material responds is equivalent to or larger than the energy of the bandgap of the material. Extrinsic semiconductors, such as doped $\mathrm{Si}$ and doped Ge can also act as photoconductors. For example, Ga-doped Ge (Ge:Ga) responds to far infrared photons with wavelengths shorter than $120 \mu \mathrm{m}$ (energies larger than $0.011 \mathrm{eV}$ ) (5) with a peak response at $-100 \mu \mathrm{m}$. Shallow level impurities, 
such as $\mathrm{Ga}$ in $\mathrm{Ge}$, are completely thermally ionized at room temperature and hence such photoconductors must operate at liquid helium temperatures so that neutral impurities are available for subsequent ionization by photon absorption. Both intrinsic and extrinsic semiconductor photoconductors must be biased with an electric field in order to move the photogenerated carriers and hence generate a detectable photocurrent. Reviews of photoconductivity in intrinsic and extrinsic semiconductors include R.H. Bube's Photoconductivity of Solids (6), R.M. Brovdy and V.J. Mazurczyck's "HgCdTe Photoconductive Detectors" (7), G.F. Stiliman, C.M. Wolfe and J.O. Dimock's "Far Infrared Photoconductivity in High Purity GaAs" (8), and P.R. Bratt's "Impurity Germanium and Silicon Infrared Detectors" (9).

Extrinsic Ge photoconductors can detect photons in the far infrared wavelength range of approximately 15-250 $\mathrm{mm}$, whereas extrinsic Si detectors respond from approximately $4 \mu \mathrm{m}$ to $30 \mu \mathrm{m}$. Ge: $2 \mathrm{n}$, Ge:8e, Ge:Ga and stressed Ge:Ga detectors respond optimally between $\sim 20-40 \mu \mathrm{m}(10), \sim 30$ $50 \mu \mathrm{m}(11),-40-120 \mu \mathrm{m}(5)$, and $-60-250 \mu \mathrm{m}(12)$, respectively. All of these detectors can be fabricated into two-dimensional arrays, except for the stressed detectors which can only be made into linear arrays. It is believed that Ge:Ga BIB photoconductors can provide detection from -30$250 \mu \mathrm{m}$ and thus may replace stressed Ge:Ga detectors and allow the construction of two-dimensional arrays. In addition to the extension in the spectrai response over the conventional (unstressed) Ge:Ga photoconductors, the BIB detector should theoretically exhibit improved responsivity due to a fixed gain of unity and also exhibit a reduction in noise from cosmic ray interference, because these devices can be made smaller in volume than conventional detectors. These three character- 
istics provide strong motivation for the development of Ge BIB photoconductors. In addition, it has been proven that BIB detectors made from extrinsic si do indeed meet their expected performance characteristics (13-17), providing further motivation for studying their Ge counterparts.

\subsubsection{BIB Detector Physics}

The theoretical operation of a $B I B$ detector is best explained in comparison to a conventional detector. ( $F$ or the sake of argument, the detectors described here shall be p-type, but the model applies equally well to n-type detectors). Figure $1(a)$ is a schematic of a conventional photoconductive detector, consisting of an infrared-active material and two degenerately doped contacts. For a Ge:Ga detector, the Ge would be doped with $\mathrm{Ga}$ to approximately $10^{14} \mathrm{~cm}^{-3}$ and have a low compensation level $\left(-10^{10}-10^{12} \mathrm{~cm}^{-3}\right.$ shaliow donors). The two degenerately doped contacts would consist of a B-implanted layer $\left\{1 \times 10^{14} \mathrm{~cm}^{-2}\right.$ at $25 \mathrm{kV}$ and $2 \times 10^{14} \mathrm{~cm}^{-2}$ at $\left.50 \mathrm{kV}\right)$ and a thin layer of metallization. The photon absorbing surface can be any one of the four sides perpendicular to the contact surfaces. In contrast, Figure $1(b)$ schematically shows a BIB detector, which consists of a pure epitaxial Ge layer grown on a heavily-doped, lightly compensated Ge substrate $\left(-10^{16} \mathrm{~cm}^{-3}\right.$ majority shallow impurities and $-1.0^{11} \mathrm{~cm}^{-3}$ minority shallow impurities). The contact on the heavily-doped layer is identical to the contacts on the conventional detector, but the contact on the epitaxial layer must be transparent to incident photons as this is the absorbing suface and thus consists of only a lightly-doped B-implanted layer $\left(-10^{13} \mathrm{~cm}^{-2}\right.$ at 


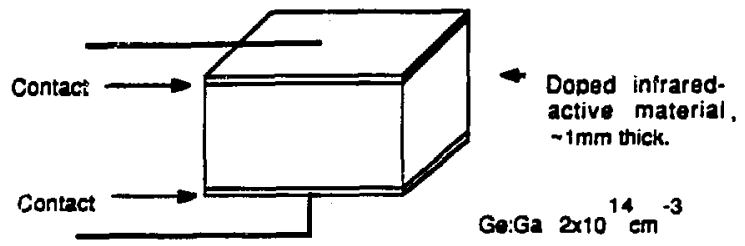

Figure 1(a). Sctiemalic of conventional detector.

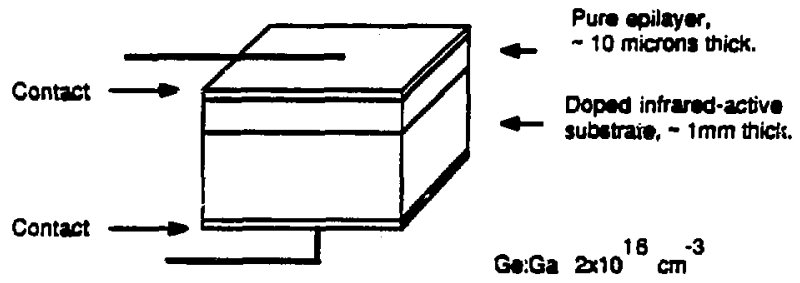

Figure 1(b). Schematic of BI3 detector.

XBL $384 \quad 1137$ 
$25 \mathrm{kV}$ ). A wire is soldered to this surface, obscurring as little of the surface area as possible. The doping levels in a Ge conventional detector as compared with Ge BIB detector are shown in Figure $2(a)$ and $2(b)$.

Like the conventional detector, the BIB detector is biased and cooled to very low temperatures during operation, but the presence of the high doping level in the IR-active layer, in conjunction with the pure epitaxial blocking layer, cause the $B I B$ detector to behave very differently from the conventional device. Its behavior is, in some ways, closer to that of a reversed biased diade, as will be described. The IR-active layer in the BIB is doped at a level high enough to result in the onset of impurity banding, but not so high as to result in a degenerate material with metallic conduction. For shallow levels in Ge the onset of impurity banding and the subsequent decrease in ionization energy occur at doping levels of approximately $5 \times 10^{15} \mathrm{~cm}^{-3}$ (18). A doping level above $3 \times 10^{17} \mathrm{~cm}^{-3}$ results in a completely degenerate layer. In addition to the heavy doping with the majority shallow impurity, the IRactive layer must have a very low concentration of compensating donors which are all ionized by an equal number of acceptors. At cryogenic liquid helium temperatures, and upon application of a bias, as shown schematically in figure 3(a), the negative charge states on the compensated acceptors are swept out of the heavily doped layer, via hopping conduction $(19,20)$ through the impurity band. Unlike a regular reversebiased diade, the electrical carriers are not transported via the conduction or valence bands, but the negative charge states are transported through the impurity band by "hopping" of holes from neutral acceptors to ionized acceptors. This hopping can occur due to the close proximity of the impurity wave functions when the doping level is high enough. Thus, 


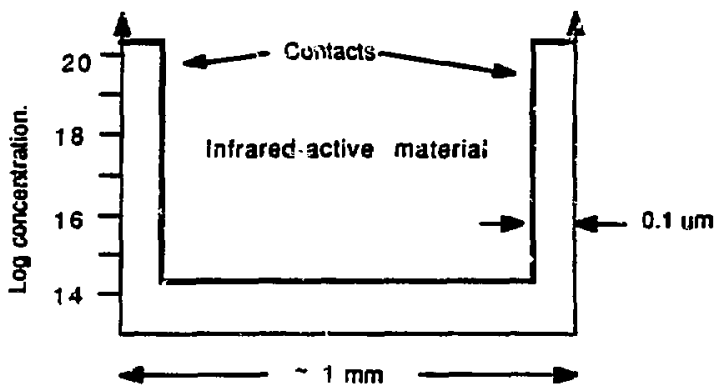

Figurs 2(a). Doping levels in a conventional Ge detector.

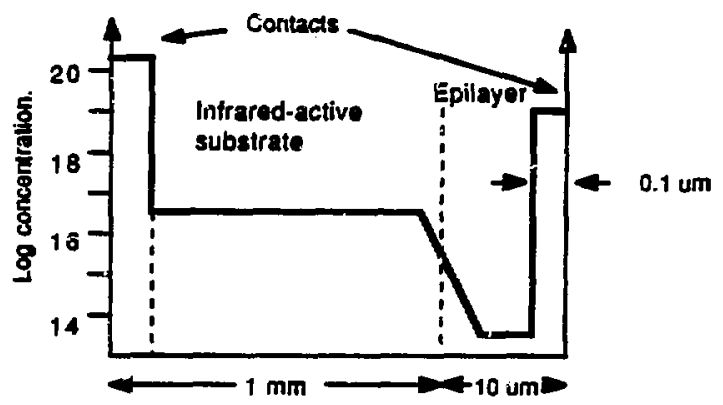

Figure 2 (b). Doping levals in a Ge BIB detecior. 


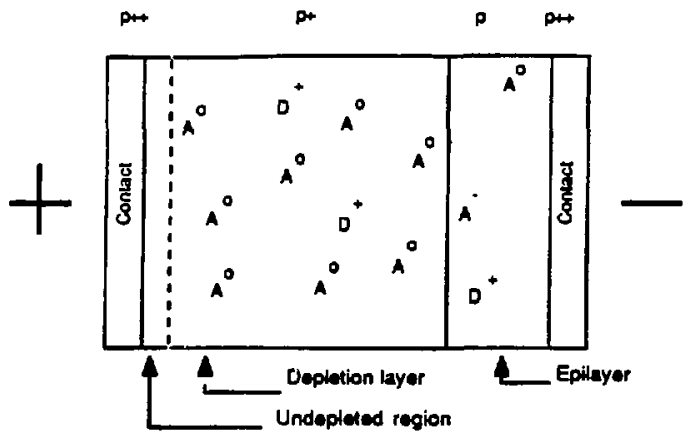

Figure 3(a). Schematic of BIB delector at low temperature and under bias, illustrating depletion layer.

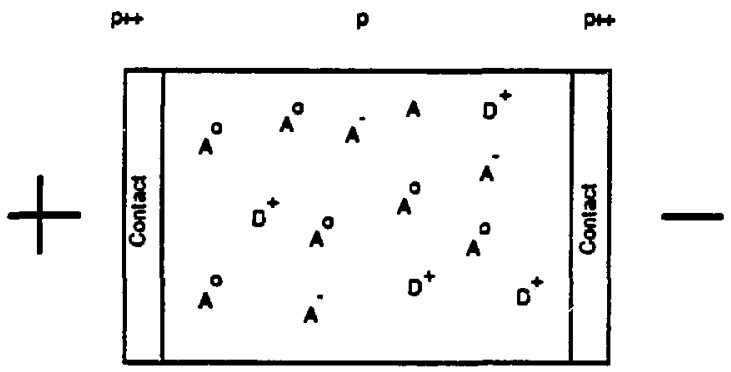

Figure 3(b). Scherintic of conventional detector at low temperature and under bias - no depletion layer. 
the negative charge states (and obviously not the impurities tnemselves) of the compensated acceptors are swept towards the positively biased contact. An equivalent way of describing this is that holes are injected at the positive contact and migrate towards the negative contact via hopping from neutral acceptors to ionized acceptors. Once the holes reach the pure epitaxial layer, however, they cannot further migrate towards the negative contact, because the epitaxial layer is pure, and thus there are too few acceptors available to transport the holes, i.e. there is no impurity band in which the holes can be transported. Furthermore, the thermal energy of the holes is too 5 mall for them to reach the valence band. The pure layer effectively blocks the current flowing in the impurity band, hence the name "blocked impurity band" detector. The iransport of the negative charge states on the compensated acceptors upon application of the bias results in a depletion region in the heavily doped layer, as shown in Figure $3($ a). This depletion region lacks ionized acceptors and thus consists only of neutral acceptors and positively charged compensating donors. The width of the depletion region depends on the applied voltage and residual minority donor concentration.

In comparison to the BIB detector, a conventional detector at low temperature and under bias is shown schematically in Figure 3(b). Conventional detectors must be doped well below the hopping conduction limit $\left(-10^{14} \mathrm{~cm}^{-3}\right)$, because there is no pure epitaxial layer to block current due to hopping conduction and hence this would be a source of undesirabie dark current in the device. ("Dark current" is current which flows in the absence of an incident photon flux and is a source of noise in the detector). Upon application of the bias, no depletion region results, there remain $-10^{14} \mathrm{~cm}^{-3}$ neutral acceptors, $-10^{11} \mathrm{~cm}^{-3}$ 
positively charged compensating donors and an equal number of negatively charged compensated acceptors.

In direct correlation to a single-sided junction (so long as the epitaxial layer is thin compared with the depletion width), the width of the depletion region in the $B I B$ device depends on the concentration of the compensating donors and the applied voltage:

$$
w=\left(2 \varepsilon_{0}\left(V_{a}-V_{b i}\right) /\left(e N_{D}\right)\right)^{i / 2}
$$

where $w$ is the uidth of the depletion region, $V_{a}$ is the applied voltage, $v_{b j}$ is the built-in voltage of the pure layer/IR-active layer junction and is of the order of the shallow acceptor energy (see Appendix I), $\varepsilon$ is the dielectric constant of the semiconductor material, $\varepsilon_{0}$ is the permittivity of vacuum, $e$ is the charge of an electron and $N_{D}$ is the concentration of compensating donors. Petroff and Stapelbroek (15) further included the thickness, $t$, of the pure epitaxial layer when calculating the depletion width:

$$
w=\left(\left(2 \varepsilon_{0}\left(V_{a}-V_{b i}\right) /\left(e N_{D}\right)\right)+t^{2}\right)^{1 / 2}-t
$$

Figure 4 details the depletion layer, space charge, electric field and potential diagran for a biased BIB detector, which is the properly biased configuration, while figure 5 is of the forward biased configuration. These schematics illustrate the ideal case of an abrupt concentration difference between the heavily doped IR-active layer and the pure epitaxial layer. (In reality, as will be shown in Section 2.2.2.2, some diffusion of the dopant element from the heavily doped layer into the 


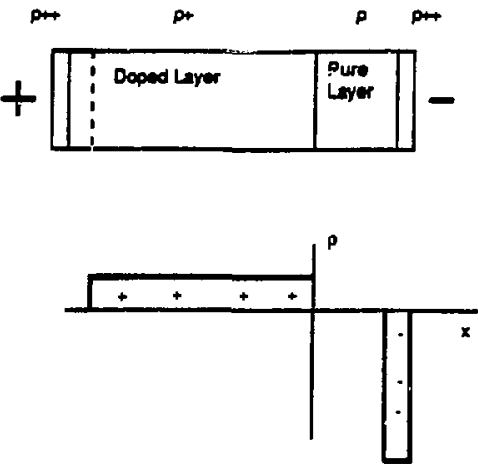

(a) Einsed datector.

(b) Soecos change. $p$, in davetor.

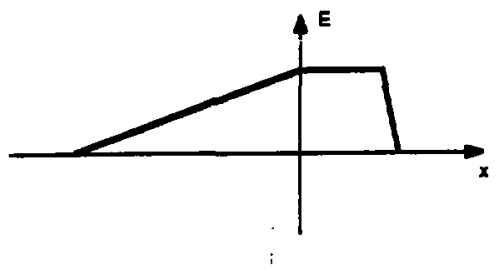

(c) Electic finde, E, in denetor.

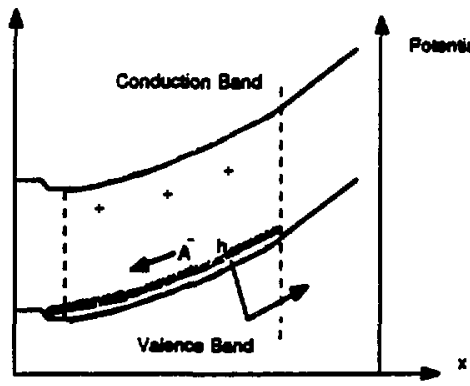

(d) Poventiel energy dicoren for bined denctur, shown with phesionizuion of a hol from a neverd cocepese in the imperity bend bo the velence bend.

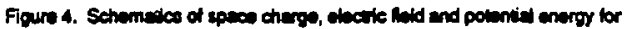

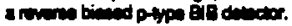




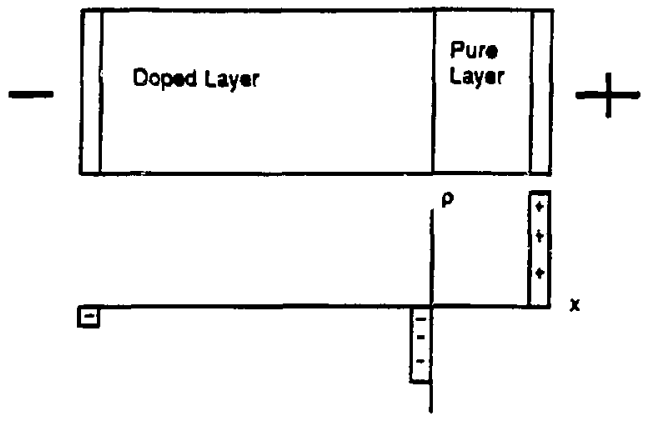

(a) Biased detector.

(b) Space charge, f.

E

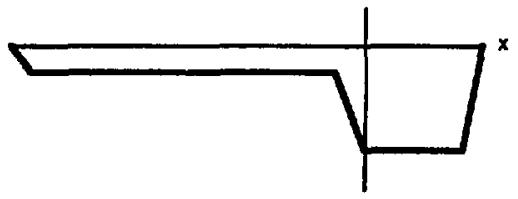

(c) Electric field, E.

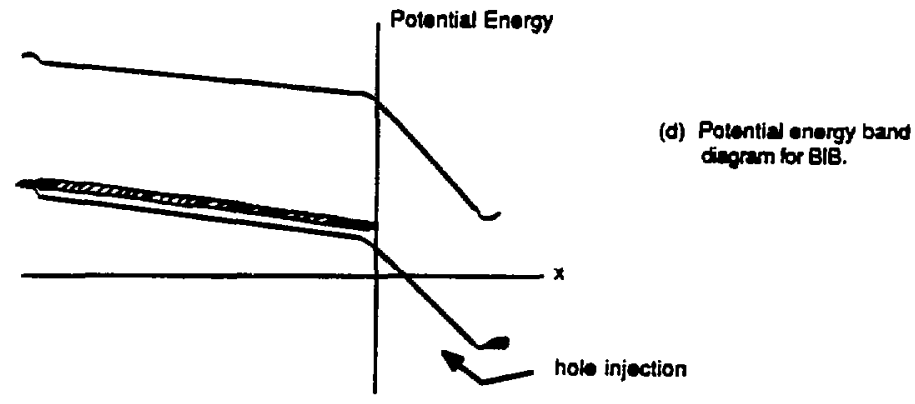

Figure 5. Schomalice of the epace charge, electric fieid and potontial energy for a fonwerd biesed p-type BIB delactor.

XEL $884 \quad 1140$ 
epitaxial layer does occur during the high temperatlires used in the epitaxial growth process). In the reverse biased case, space charge is assumed to only build up in the depletion region of the heavily doped ayer and in the negatively biased contact region, as shown in Figure $4(b)$. If there is any undepleted region in the IR-active layer, there will be no electric field in this region and hence any holes generated there from photon absorption will not be collected. It is desirable that the depletion region be large compared with the undepleted region and the epitaxial layer so that the majority of photon absorption occurs there. As is obvious from the above equations, the applied voltage, residual donor concentration and epilayer thickness must all be balanced in order to optimize the depletion width.

The photon absorption process in a BIB detector is shown in figure 4(d). A photon of energy equal to, or greater than, the ionization energy of a neutral acceptor is absorbed, releasing a hole into the valence band and creating an ionized acceptor. The hole is transported in the valence band towards the negatively charged contact, while the negative charge state on the fonized acceptor is swept towards the positive contact in the impurity band via hopping conduction. It is important to emphasize that the photo-generated hole, migrating through the valence band, can pass through the pure "blocking" layer, while a hole migrating through the impurity band via hopping conduction is effectively blocked by the pure epitaxial layer. Thus, the pure layer blocks the dark current, but passes the photocurrent. The depletion region shown in Figure 4 (a) is devoid of $A^{-}$centers, and hence the photo-generated hole cannot recombine with an ionized acceptor on its way towards the positive contact. (There undoubtedly will be some residual compensated acceptors 
in the "pure" epilayer, and thus the hole could recombine in this layer, but ideally the epilayer will be thin enough so that this effect is negligible). In the ideal case no recombination events occur and there is one hole and one negative charge collected per ionization event. The gain of the detector, which can be defined as "the collection length over which the charge travels (both the free hole and the $A^{-}$state), divided by the total detector length" $(21)$, is equal to unity when the depletion region extends the length of the detector. The fixed gain results in the elimination of generation-recanbination noise, which occurs in conventional detectors where the gain varies from photoionization event to photoionization event (22). In conventional detectors, the photogenerated hole will recombine with an ionized acceptor in its path on the way to the negative contact, because there are ionized acceptors distributed throughout the IR-active region. The gain in a conventional detector thus depends on how far the carrier travels in relation to the total detector length, and this depends on where the carrier is generated and where it recombines. Thus, these detectors exhibit generation-recombination noise.

The existence of the high doping level, in conjunction with the pure epitaxial layer in a BIB detector, yields two advantages in addition to the aforementioned elimination of generation-recombination noise. The detector volume can be reduced without compramising photoabsorption, because the absorption is, tc first order, directly proportional to the concentration of impurities: 
where $a$ is the linear absorption coefficient, $\sigma$ is the photon capture cross-section and $N$ is the concentration of the neutral majority shallow impurities. A smaller volume means there is less bulk Ge available to absorb high energy radiation from cosmic rays in a space environment, which is a source of interference signal in the detector.

The level of doping is high enough such that an impurity band begins to form, thereby decreasing the energy for photo-ionization (18). The decrease in ionization energy results in an increase in the detectable photon wavelength. In conventional detectors, the shallowest levels are ionized at $-.010 \mathrm{eV}$, corresponding to a maximum detectable wavelength of -125 uril. With impurity banding, the ionization energy decreases, which increases the wavelength of the detector onset. This increase in the waverength cutoff has also been achieved with stressed detectors $(5,12)$, however, the ability to make the desirable two-dimensional detector arrays from stressed detectors is severely limited by the mechanical stress apparatus.

In brief, the three improvements of BIB detectors over conventional detectors are: 1) elimination of generation-recombination noise, 2) a reduction in cosmic ray interference due to a reduction in detector volume, and 3) an increase in the maximum wavelength of the detector response.

In summary, the optimum IR-active layer will contain a low level of residual compensating shallow donors so as to maximize the depletion region width and thus maximize the volume available for photon absorptioil. The optimum doping level of the majority shallow acceptor is not known, but must be within the impurity banding regime. In addition, the IRactive material must have as low a dislocation density as possible to 
minimize the number of traps and scattering centers. Single crystal, low dislocation density germanium with a very low compensation level and the required high doping level is grown in our laboratories and hence its avałlability was not an issue. Such heavily-doped Ge can also be purchased commercially, however, the concentration of compensating impurities is typically 10-1000 times higher than that grown by us.

For the epitaxial layer to function effectively as a blocking layer, it must have the following characteristics:

1) Low dislocation density (as low as possible), to minimize the number of deep traps which may act as trapping centers for the photogenerated holes and possibly as generation centers for dark current.

2). Total shallow impurity concentration well below the hopping conduction limit, $<10^{14} \mathrm{~cm}^{-3}$, to effectively block leakage current from the heavily doped layer.

3) P-type for a p-type IR-active layer (n-type for an n-type IRactive layer) to minimize the potential barrier at the substrate/epilayer interface.

4) The interface region must have a low density of interface states, which may arise during imperfect epitaxial growth, to minimize the number of deep traps in this region.

The growth of a pure and structurally perfect Ge epitaxial layer, which would serve as the blocking layer in a Ge BIB detector, was not a technology previously developed anywhere and thus the growth of this epitaxial layer was the first step to be accomplished in the fabrication of Ge BIB detectors. The development of the Ge epitaxial technique and subsequent layer characterization constitutes a substantial part of the work to be presented here. 
1.2.2 Review of Silicon and Germanium BIB Detectors

The concept of the BIB detector was first introduced by M.D. Petroff and M.G. Stapelbroek of Rockwell International (Thousand Oaks, California) in 1977 (23). The performance characteristics of the first silicon BIB devices were presented in 1980 at the NOSC Conference on Extrinsic Silicon Detector Behavior (24) and also at the IRIS Detector Specialty Group Meeting (25), by M.D. Petroff and M.G. Stapelbroek. The researchers at Rockwell continued to study Si BIB detectors in subsequent years, finding them to indeed meet many of their anticipated performance characteristics (13-17). In 1984, W.A. Kleinhans, M.D. Petroff and M.F. Stapelbroek presented the first data for Si BIB arrays (26) and have since continued to develop them (27).

The Rockwell Si BIB detectors consist essentially of two epitaxial layers deposited on a degenerately doped $n$-type Si substrate. The first layer is a heavily As-doped $\left(4 \times 10^{17} \mathrm{~cm}^{-3}\right)$ IR-active layer with a residual boron concentration of approximately $5 \times 10^{13} \mathrm{~cm}^{-3}$. The subsequent Si layer is not intentionally doped and is as pure as possible to serve as the blocking layer. The surface of this second layer has an implanted transparent contact. Petroff and Stapelbroek have evaluated such Si BIB detectors theoretically and experimentally (15) and have proposed a model for carrier transport in the IR-active layer based on phonon-assisted tunneling, or hopping, of electrons between neutral impurity sites and compensated fonized impurity sites. Fram this analysis they obtain:

1) The effective free mobility of hopping carriers as a function of majority impurity concentration, 
?) The collection time of the ionized impurities and carriers created by photon absorption,

3) The frequency dependence of the gain,

4) The detector response as a function of applied bias.

In this same paper (15), Petroff and Stapelbroek present experimental detector data for photocurrent and dark current as functions of applieo bias, as compared with the theoretical values. At intermediate dias values the experimental and theoreticsl values for current are very similar. There is also a close correlation between the experimentai ano theoretical values for gain and quantum efficiency as functions of applied bias. (All of the data were collected at a photon flux of $1.5 \times 10^{12} \mathrm{phcm}^{-2} \mathrm{~s}^{-1}$, a temperature of $10 \mathrm{~K}$, and an incident wavelength of $20 \mu \mathrm{m})$. By measuring the background-limited noise current as a function of bias, Petroff and Stapelbroek found that the gain is indeed unity at intermediate bias levels, as predicted for BIB detectors. The quantum efficiency depends on the depletion layer thickness, which is bias-dependent at low fields until the entire IR-active layer is oepleted, and the simple noise theory for reverse-biased photodiodes is directly applicable to BIB detectors so long as the depletion width is less than or equal to the IR-active layer thickness. When the depletion region extends into the contact region, sources of noise other than shot noise may begin to become significant and the simple noise theory no longer holds. They also propose that leakage of impurity band carriers alang structural imperfections in the blocking layer may be a source of noise. In a separate paper, Stapelbroek, Petroff, Speer and Bharat (16) discuss the origins of excess low frequency noise $(<100 \mathrm{~Hz}$ ) at intermediate IR 
backgrounds $\left(10^{14}-10^{15}\right.$ phem $\left.^{-2} \mathrm{~s}^{-1}\right)$, attributing this noise to fluctuatiors in the blocking layer space charge.

In response to the presumed need for a theoretical model which is more transparent than the statistical Monte Carlo method used by Petroff and Stapelbroek (15), Szmulowicz and Madarz published an analytical model which they apply to the Si:As BIB detector (28). They calculated gain, quantum efficiency, excess noise factor, responsivity and detectivity and showed that all of these parameters are sensitive functions of applied bias and compensating minority impurity concentration both of which directly affect the depletion layer thickness.

The early successes of the discrete Si BIB detectors led to Si BIB arrays which have shown high sensitivity and quantum efficiency in the long wavelength spectral region (to $28 \mathrm{\mu m}$ ) as well as wide frequency response, low optical cross-talk, nuclear radiation hardness and stable predictable performance (27). Arrays will not be further discussed here, as it is beyond the scope of this work.

As discussed, Si BIB technology is approximately ten years old and has received a relatively concentrated research effort as compared with Ge BIB work. Si BIB detectors are of more interest to the military conmunity than are Ge BIB detectors and hence have received more attention by researchers than their Ge counterparts. As discussed in a previous section, Ge BIB detectors are primarily of interest to astrsnomers who seek information about astrophysical galactic infrared sources from $-30-250 \mu \mathrm{m}$. The first published Ge BIB detector data appeared in 1985 by Hadek, Farhoomad, Beichman, Watson and Jack (29). Their Ge BIB device was a layered structure consisting primarily of a $12 \mathrm{um}$ thick IR-active layer of Ge doped with boron at a level of $3 \times 10^{16} \mathrm{~cm}^{-3}$, a 1 um thick 
undoped Ge layer and degenerate contacts. The IR-active layer and the undoped layer were grown by chemical vapor deposition using $\mathrm{GeCl}_{4}$ as the gas precursor, on a degenerately doped Ge substrate. Hadek, et. al. report the relative response of these detectors at $1.7 \mathrm{~K}$ as a function of incident photon wavelength (29). The BIB detectors exhibited a maximum wavelength cutoff of approximately $200 \mathrm{\mu m}$, which is an increase of about $75 \mu \mathrm{m}$ over a conventional detector with a lower doping level. The peak response occurred at $\sim 90 \mu \mathrm{m}$, which is comparable to a conventional detector. These first BIB detectors were very noisy and exhibited a $10^{-6} \mathrm{~A}$ dark current at an unspecified bias, compared with the approximately $10^{-12}-10^{-16}$ A dark current observed for a conventional detector. For the device to function optimally, the dark currents would have to be decreased by many orders of magnitude. Hadek subsequently published an analysis of the theoretical charge distribution and response time for a Ge:B BIB detector (30). He detailed the conditions required for an optimum depletion region width and examined the electric field distribution in the device, both as functions of applied voltage, blocking and doped layer thicknesses, and majority and minority impurity concentrations in the IR-active layer. Taking values for a typical detector, with the concentration of minority impurities equal to $5 \times 10^{13} \mathrm{~cm}^{-3}$, majority impurity of $5 \times 10^{16} \mathrm{~cm}^{-3}$ and an electric field of 10-100 $\mathrm{Vcm}^{-1}$, then the depletion width was on the order of $10 \mu \mathrm{m}$ for a $12 \mu \mathrm{m}$ thick IR-active layer with a $1 \mathrm{\mu m}$ thick blocking layer. The response time for such a device was caiculated to be on the order of $10^{-8}-10^{-9} \mathrm{~s}$. Hadek did not present any experimental data which would support his theoretical analysis.

The Ge BIB detector work was continued by D.W. Watson of the Cali- 
fornia Institute of Technology in Pasadena, California and by J.E. Huffman of Rockwell International, Anaheim, California, resulting in a paper submitted to Applied Physics Letters in 1987 (31). This was the first work which reported relatively extensive experimental data on Ge:Ga BIB detectors, although some important infarmation - which will be discussed shortly - had been omitted. These detectors consisted primarily of a pure epitaxial Ge layer grown on an IR-active Ge substrate which was doped with Ga at a level of $-3 \times 10^{16} \mathrm{~cm}^{-3}$. The epitaxial layer was grown via chemicai vapor deposition using $\mathrm{GeCl}_{4}$ gas. Watson and Huffman reported the following experimental results from these BIB detectors:

1) Absolute responsivity (in $\mathrm{AW}^{-1}$ ) of a Ge BIB detector at $1.7 \mathrm{~K}$ compared with an unstressed conventional Ge:Ga photoconductor at $4.2 \mathrm{~K}$, as a function of incidant photon wavelength: The Ge:Ga photoconductor cut off at $-120 \mu \mathrm{m}$, as was expected, while the Ge BIB detector cut off at $-190 \mu \mathrm{m}$, as would be expected for the heayy doping levels in the IRactive layer. The responsivity of the BIB was $-5 \mathrm{AW}^{-1}$ at its peak of $-140 \mu \mathrm{m}$, compared to $5 \mathrm{AW}^{-1}$ at $110 \mu \mathrm{m}$ for the conventional detector.

2) Respensivity as a function of bias: The BIB detector exhibited essentially no responsivity in the forward biased configuration and $-3 \mathrm{AW}^{-1}$ at $0,0 \mathrm{mV}$ in the reverse biased configuration, which is the properly biased configuration for a BIB detector.

3) Capacitance as a function of bias: The capacitance of the BIB detector was -140 pf at $0 \mathrm{mV}$ bias and decreased as the bias increased (in both the forward and reverse bias directions) as would be expected, because the depletion region width increased as the bias increased unti? breakdown voltages of $-40 \mathrm{mV}$ and $+25 \mathrm{mV}$ were reached.

4) DC current as a function of applied bias: A value of "zero" 
nanoamperes was reported for bias values between $-40 \mathrm{mV}$ and $+25 \mathrm{mV}$. The DC current increased (negatively) at biases $>25 \mathrm{mV}$ and increased (positively) at biases $<-40 \mathrm{mV}$. The problem with reporting the $\mathrm{OC}$ current data in this fashion is that "zero" nanoamperes is only an upper limit and it is not clear what the real values were. Optimum conventional photoconductors exhibit $D C$ currents on the order of $10^{-16}-10^{-17} \mathrm{~A}$ in the dark and so Ge $8 I 8$ detectors which exhibit dark currents less than $10^{-9} \mathrm{~A}$ are far from optimum and are essentially useless when operated under the law background conditions for which they are intended.

Watson and Huffman's Ge BIB detectors show great promise in terms of responsivity, spectral response and quantum efficiency, but the authors fail to convince the reader that the noise and dark current characteristics are in the range of "pronising". In addition, they have not yet published data regarding the physical and electronic properties of the Ge epitaxial blocking layer, although it has been implied that these will be published in the near future (31).

From this brief review of the current status of Ge:Ga BIB detectors, the following conclusions are drawn:

1) When the IR-active substrate is doped with Ga at a concentration of $2 \times 10^{16} \mathrm{~cm}^{-3}$ the detector cutoff wavelength is $-190 \mu \mathrm{m}$, an increase of $-65 \mu \mathrm{m}$ over conventional Ge:Ga detectors. However, at these doping levels and at th. temperatures studied to date $(1.7 \mathrm{~K}$ minimum) the dark current is very high. If the epitaxial layer is truly acting as a blocking layer, and if the dark current is due to thermally ionized carriers from the heavily doped layer, then the operating temperature for these detectors will have to be less than $1.7 \mathrm{~K}$ in order to reduce the number of thermaliy ionized carriers. If the dark current cannot be reduced to 
reasonable levels, the doping level in the IR-active layer will have to be reduced, thus reducing the dark current but also compromising the detector cutoff wavelength. The detector perfomance at temperatures less than $1.7 \mathrm{~K}$ needs to be studied.

2) Little, or nothing is known about the effect of the blocking layer properties on the detector performance. For example, it is not known to what extent the residual impurity concentration or structural defect concentration affect the "blocking" characteristics of the epilayer. In addition, the cptimum epilayer thickness is not known. The blocking layer must be thick enough to prevent tunnelling of carriers from the impurity band of the IR-active layer into the valence band of the epilayer, but it must also be thin enough to minimize photon absorption is this layer and thin enough so that a minimum of the voltage drop occurs across this layer. These remarks apply to Si BIB detectors as well.

3) The responsivity, quantum effeciency and gain of Watson and Huffman's BIB detectors are fairly respectable in comparison to conventional detectors, but could probably be improved with further optimization of the material parameters.

\subsection{Semiconductor Epitaxy}

\subsection{Introduction to Semiconductor Epitaxy}

Semiconductors can be grown epitaxially on a variety of materials using a number of techniques which possess such names as Physical Vapor Deposition (PVD), Vapor Phase Epitaxy (VPE), Liquid Phase Epitaxy (LPE), 
Solid Phase Epitaxy (SPE), Chemical Vapor Deposition (CVO), Molecuiar Beam Epitaxy (MBE), Metalorganic CVD (MOCVO), and Chemical Beam Epitaxy (CBE). Reviews of such techniques are given in several references (3234). Recently, some of these methods have been refined leading to techniques called, for example, Low Pressure CVD (LPCVD) (35) and Plasma Enhanced CVO (PECVO) (36).

The literature contains some information about Ge epitaxy, but not nearly so much as concerning Si epitaxy, as might be expected. Ge has been deposited using the techniques of PVO (37-49); CVD using $\mathrm{GeI}_{2}$ (50, $51), \mathrm{GeCl}_{4}(52,53)$ and $\mathrm{GeH}_{4}(54-63)$ as the gas precursors for Ge; LPE (64-67); SPE $(68,69)$; and MBE (70). Of all the Ge epitaxial layers grown using the above techniques, none were reported to be of the purity required for the present work, although some showed promise and many were of the required crystalline perfection. In addition to the lack of purity, there was very little data available regarding the details of deposition or the electronic and physical properties of the layers, nor was a wide range of deposition parameters explored. The majority of the papers mereiy presented an exploratory study of the feasability of depositing Ge epitaxially on specific substrates, not necessarily Ge, using a specific technique.

When choosing a technique to produce the Ge layers for this work, it was established that the technique must fulfill the following requirements:

i) It must be capable of producing very pure Ge layers.

2) It must be inherentiy simple in equipment design and process to minimize the risk of introducing contaminants and to allow a quick exploration of the deposition parameters. 
3) It would be preferable that this technique be already proven to produce good quality Ge epitaxial layers - either homoepitaxially or heteroepitaxially.

Physical Vapor Deposition (PVD) was eliminated as a possible technique for this work based on the following reasons:

1) No published work had yet produced relatively pure Ge or Si layers.

2) The PVD techniques, such as vacuum evaporation and sputtering, typically produce layers with high point defect densities. Although not proven, it was thought the defect density might be a problem in producing layers of the structural perfection required of BIB detectors. LPE is capable of producing high quality epilayers, providing that there is an element which dissolves sufficient Ge, but is by itself not an electrically active dopant. Ge-Pb, Ge-In, Ge-Sn and Ge-Ga alloys are liquids at low temperatures (e.g. approximately 5 percent Ge dissolved in $\mathrm{Pb}, \mathrm{In}$, Sn and Ga melts at $-750^{\circ} \mathrm{C},-450^{\circ} \mathrm{C},-400^{\circ} \mathrm{C}$ and $-300{ }^{\circ} \mathrm{C}$, respectively (71)) and have been used to produce Ge epitaxial layers (64-67). However, both In and Ga are shallow acceptors in Ge and their solid solubility limits in $\mathrm{Ge}$ are $2 \times 10^{18} \mathrm{~cm}^{-3}$ at $450^{\circ} \mathrm{C}$ and $3 \times 10^{20}$ $\mathrm{cm}^{-3}$ at $300^{\circ} \mathrm{C}$, respectively $(72)$, leaving an undesirably high level of electrically active contaminants in the Ge layer. Sn and $\mathrm{Pb}$ are isoelectronic with $\mathrm{Ge}$ and would not produce electrically active impurities, but the literature regarding the use of these elements in Ge LPE is scant, and the purities reported were not of the order required of the BIB detector epitaxial layer. 
SPE has been used to grow Ge from Al metal layers containing Ge at the solid solubility limit $(68,69)$. However, the resulting Ge layers are p-type with a residual Al concentration on the order of $3 \times 10^{15} \mathrm{~cm}^{-3}$ (69), which is too high for the layers required for this work.

In studies employing the CVD technique using Ge halogen compounds, the highest purity layers reported using $\mathrm{Gel}_{2}$ contained $5 \times 10^{14} \mathrm{~cm}^{-3}$ to $4 \times 10^{15} \mathrm{~cm}^{-3}$ shallow impurities (51). Recently, Watson and Huffman have deposited $\mathrm{Ge}$ layers using $\mathrm{GeCl}_{4}$ with a net shallow acceptor concentration of $-2 \times 10^{13} \mathrm{~cm}^{-3}$, measured using a room temperature spreading resistance technique (31). Despite these encouraging results, the halogen compounds were rejected for this work due to their potential reactivity with the deposition chamber materials and doped substrate (autodoping), which could introduce contaminants and dopant impurities in the growing epilayer.

Papazian and Reisman reported growth of As-doped Ge epilayers on GaAs with a residual shallow acceptor concentration of $5 \times 10^{14} \mathrm{~cm}^{-3}$ using $\mathrm{GeH}_{4}$ gas as the precursor for CVD (54). Other researchers have reported high quality Ge epilayers grown via CVD of $\mathrm{GeH}_{4}$ on Ge $(56,60,61)$, on GaAs $(55,60)$, on $\mathrm{Si}(57-59)$, on glass (63) and on $\mathrm{NaCl}(62)$, although none report layers as pure as those of Papazian and Reisman. The CVD technique using $\mathrm{GeH}_{4}$ was ultimately chosen as the epitaxy technique for this work, based on the simplicity of the technique and the corresponding inherent purity adrantages, and also because of the promising results of Papazian and Reisman. Additional reasons for choosing CVD using $\mathrm{GeH}_{4}$ were the availability of semiconductor grade $\mathrm{GeH}_{4}$ gas and the large amount of information in the literature regarding high quality silicon epitaxial layers grown via CVD using $\mathrm{SiH}_{4}$ gas - a system very similar 
to the $\mathrm{Ge} / \mathrm{GeH}_{4}$ system. A sampling of the literature available on Si epitaxial layers grown via $\mathrm{CVO}$ of $\mathrm{SiH}_{4}$ is given in the references $(73-90)$.

The details of the specific CVD apparatus and process used for this work are described in the following section. Also, considerations of the effects of specific CVD paraneters on the epilayer growth and quality are discussed where appropriate in the following section.

\subsection{Germanium Epitaxy}

As discussed above, the method chosen to deposit epitaxial Ge layers on Ge substrates was that of CVO using $\mathrm{GeH}_{4}$ as the source gas. Two types of CVD systems were built and used for growing the epilayers: The first was a vertical CVD chamber, while the second was a horizontal CVD chamber. Both the vertical and horizontal chambers were used only for atmospheric pressure depositions. The decision to build the horizontal chamber came after taking the vertical chamber to its "limits", when it became clear that certain changes were necessary in order to achieve the goals of epilayer purity. The vertical CVD chamber and the layers produced using it will be discussed first, followed by a discussion of the epilayers grown using the horizontal chamber.

\subsubsection{Epitaxy using the Vertical CVD Chamber}

\subsubsection{Apparatus and Epitaxy Procedure}

The vertical CVo chamber is shown in the schematic of Figure 6 and in the photograph of Figure 7. A brief description of the apparatus and its 


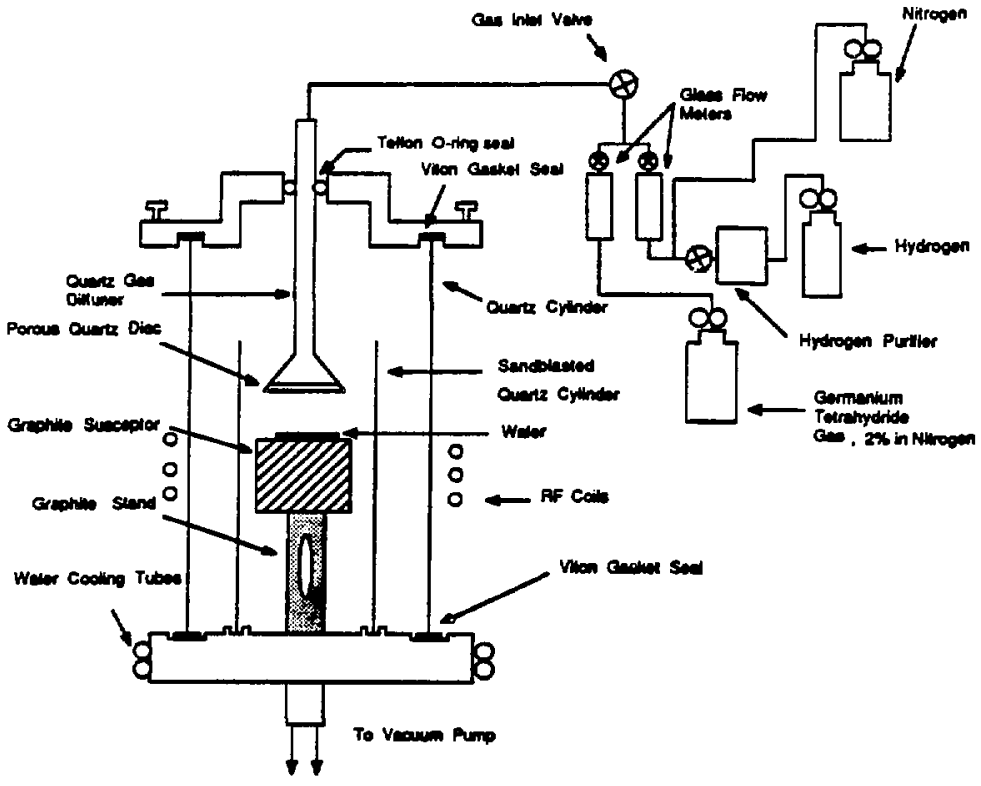

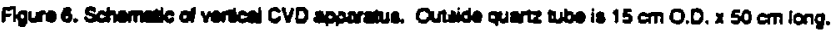




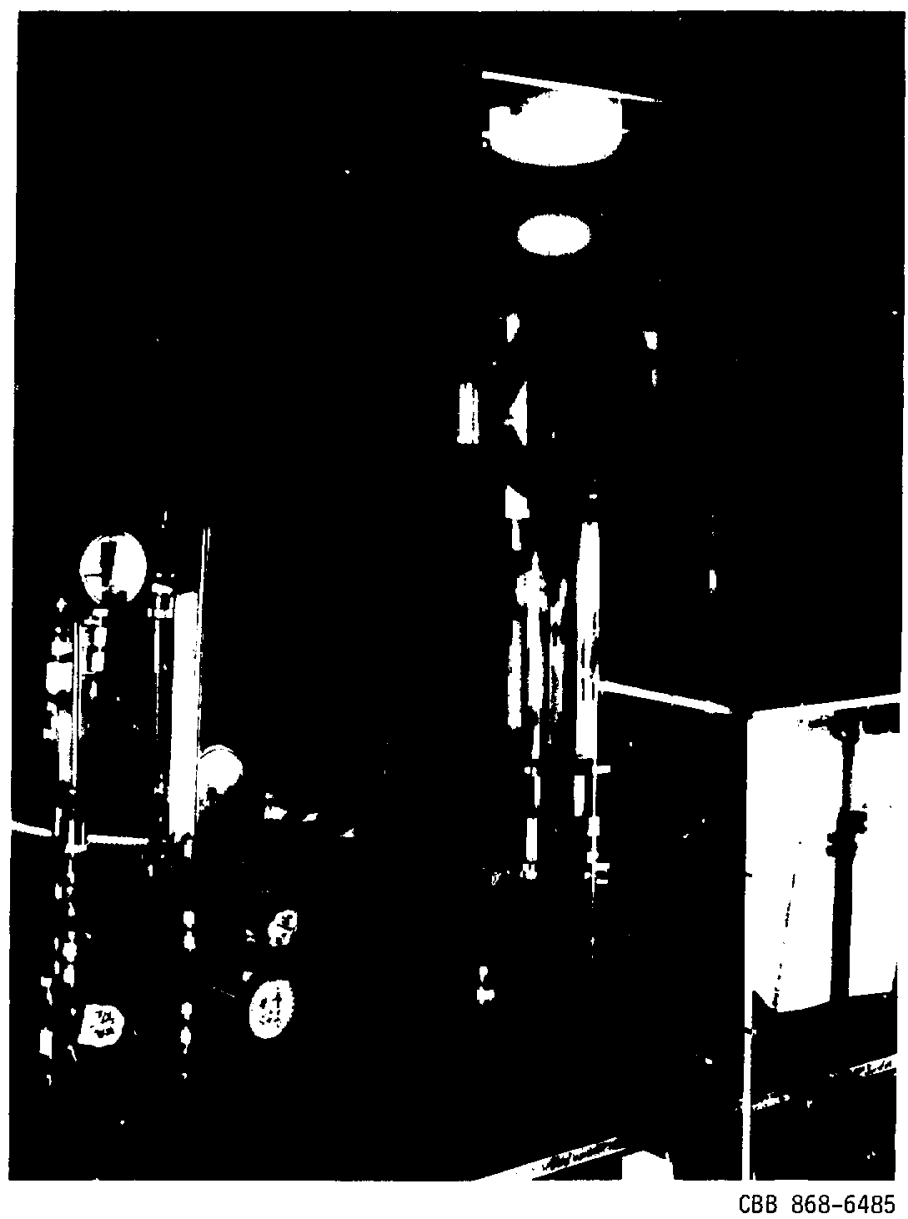

Figure 7. Photograph of vertical CVD apparatus. 
components is as follows: The main body of the chamber was a $15 \mathrm{~cm}$ diameter quartz tube, inside which was another quartz tube and a $7.5 \mathrm{~cm}$ diameter cylindrical graphite susceptor which held the Ge wafers. The susceptor was inductively heated using an $R F$ generator ${ }^{1}$ and baked at $950^{\circ} \mathrm{C}$ in $\mathrm{N}_{2}$ for two hours prior to use. The outer quartz tube was sealed on both ends using flat viton ${ }^{2}$ gaskets fixed in aluminum plates and the quartz gas inlet tube sealed to the top aluminum plate with a Teflon ${ }^{3}$ oring. The gas flow rates were monitored with glass flow meters ${ }^{4}$. The $\mathrm{H}_{2}$ gas passed through a purifier ${ }^{5}$ prior to entering the flow meter, while the $\mathrm{N}_{2}$ gas and $\mathrm{GeH}_{4}^{6}$ gas were not purified. Stainless steel tubing and stainless steel vacuum fittings ${ }^{7}$ were used throughout the system. The aluminum and quartz components were chemically etched and the stainless tubing used for the gas lines was heated with a hand-held hot air gun prior to use.

The orientations, doping elements and doping concentrations of the Ge substrates used for epitaxy are described in Table I. The substrate wafers were 7 apped with $9_{\mu} \mathrm{m} \mathrm{Al}_{2} \mathrm{O}_{3}$ powder and polished to an optical finish with a colloidal $\mathrm{SiO}_{2}$ and $\mathrm{H}_{2} \mathrm{O}_{2}$ solution ${ }^{8}$. The dislocation density in all the substrate wafers, prior to deposition, was $-10^{3} \mathrm{~cm}^{-2}$.

1. Generater from Lepel High Frequency Laboratories, Inc, New York, Niew York, madel $T-5-3-K C-A-B$.

2. Low vapor pressure fluoroelastomer from DuPont, Wilmington, Delaware.

3. Fluorocarbon material fram DuPont, Wilmington, Delaware.

4. Models 602 and $603 \mathrm{glass}$ flow meters from Mathes on Gas Products, East Rutherf ord, New Jersey.

5. $\mathrm{H}_{2}$ purifier from Englehard Industries, Inc., Newark, New Jersey.

6. Two percent $\mathrm{GeH}_{4}$ in $\mathrm{N}_{2}$ from Mathes on Gas Product, East Rutherford, New Jersey.

7. Swagelock vacuum fittings from Crawford Fitting Co., Solon, Ohio.

8. Cab-0-sperse colloidal silica dispersion $5 C \times 2$ grade from Cabot Corp., Tuscola, Illinois. Polishing solution was $300 \mathrm{ml}$ colloidal silica, $300 \mathrm{ml} \mathrm{H}_{2} \mathrm{O}$ and $200 \mathrm{ml} \mathrm{H}_{2} \mathrm{O}_{2}$. 
Table I.

Sample Substrate $\mathrm{H}_{2} \quad \mathrm{GeH}_{4}{ }^{*}$ Growth Film Vol.r Film No. conc. $\mathrm{cm}^{-3} 1 \mathrm{~min}^{-1} \mathrm{~cm}^{3} \mathrm{~min}^{-1}$ temp. ${ }^{\circ} \mathrm{C}$ type $\mathrm{GeH}_{4}$ thick.,

\begin{tabular}{|c|c|c|c|c|c|c|c|}
\hline I-1 & $\begin{array}{l}\text { Ge:P }(113) \\
8 \times 10^{1} 3^{-13}\end{array}$ & 5 & 210 & $700-720$ & $n$ & 0.08 & 4 \\
\hline I -2 & $\begin{array}{l}\text { Ge:P }(113) \\
9 \times 10^{13}\end{array}$ & 5 & 210 & $700-720$ & $p$ & 0.08 & 8 \\
\hline $1-3$ & $\begin{array}{l}\text { Ge:P }(113) \\
9 \times 10^{13}\end{array}$ & 2.75 & 520 & $715-725$ & p & 0.3 & 0 \\
\hline \multirow[t]{2}{*}{$I-5$} & $\begin{array}{l}\text { Ge:As }(100) \\
3 \times 10^{17}\end{array}$ & 2.75 & 400 & $595-615$ & $p$ & 0.25 & 37 \\
\hline & $\begin{array}{l}\mathrm{Ge}: \mathrm{Be}(113) \\
5 \times 10^{15}\end{array}$ & & & & $p$ & & 19 \\
\hline \multirow[t]{2}{*}{$I-6$} & $\begin{array}{l}\text { Ge:P }(113) \\
5 \times 10^{14}\end{array}$ & 2.75 & 400 & $650-670$ & $p$ & 0.25 & 10 \\
\hline & $\begin{array}{l}\mathrm{Ge}: \mathrm{Be}(113) \\
5 \times 10^{15}\end{array}$ & & & & $p$ & & 9 \\
\hline \multirow[t]{2}{*}{$\mathrm{I}-7$} & $\begin{array}{l}\text { Ge:P } \\
8 \times 10^{14}\end{array}$ & 2.75 & 400 & $695-705$ & $p$ & 0.25 & 5 \\
\hline & $\begin{array}{l}\text { Ge:Be }(113) \\
5 \times 10^{15}\end{array}$ & & & & $p$ & & 5 \\
\hline $1-13$ & $\begin{array}{l}\text { Ge:Sb }(100) \\
8 \times 10^{14}\end{array}$ & 2.75 & 400 & $720-725$ & $p$ & 0.25 & 5 \\
\hline I-14 & $\begin{array}{l}\mathrm{Ge}: \mathrm{Sb}(100) \\
8 \times 10^{14}\end{array}$ & 2.75 & 400 & $720-725$ & p & 0.25 & 8 \\
\hline \multirow[t]{2}{*}{$I-17$} & $\begin{array}{l}\text { Ge:A1 (113) } \\
1 \times 10^{11}\end{array}$ & 2.75 & 210 & $720-725$ & $p$ & 0.13 & 3 \\
\hline & $\begin{array}{l}\text { Ge:Sb }(100) \\
\text { Bxi } 14\end{array}$ & & & & $\mathrm{p}$ & & 3 \\
\hline $1-18$ & $\begin{array}{l}\text { Ge:Al (113) } \\
1 \times 1011\end{array}$ & 2.75 & 210 & $720-725$ & $p$ & 0.13 & 0.1 \\
\hline
\end{tabular}

*Two percent $\mathrm{GeH}_{4}$ in $\mathrm{N}_{2}$. 
The Ge substrate cleaning procedure was optimized during the course of several depositions. The original procedure was to boil the wafers in tetrachloroethane (TCA) twice, for 3 minutes, after removing the wafers from the polishing block; methanol rinse; 20 second etch in 20:1 $\mathrm{HMO}_{3}$ : $\mathrm{HF}$; methanol rinse; blow dry with $\mathrm{N}_{2}$. The cleaning procedure was later improved by introducing an etching step which ensured removal of the support wax used during the folishing procedure. After the wax was visually removed by boiling in TCA, the polished surfaces were masked with plastic tape and the entire wafer was etched in a $\mathrm{HNO}_{3}$ solution ${ }^{1}$ for one minute. After removal of the tape, the wafers were again boiled in TCA twice for 3 minutes, rinsed in methanol, etched in the 20:1 $\mathrm{HNO}_{\mathrm{g}}$ :HF solution for 20 seconds, rinsed with distilled methanol and blown dry with $\mathrm{N}_{2}$. The optical micrographs in Figure 8 show the effect of the cleaning procedures on the visual appearance of the epilayers. Figure 8(a) shows the visually imperfect topography of an epilayer grown on a substrate which underwent the original cleaning procedure, while Figure $8(b)$ shows an epilayer free of visual defects which was grown on a substrate cleaned using the optimized procedure. The growth conditions were similar and substrate orientations the same in both cases.

The epitaxial layers were deposited under a variety of conditions, with substrate temperatures ranging from $595-725^{\circ} \mathrm{C}, \mathrm{H}_{2}$ flow rates of 2.75-5 $1 \mathrm{~min}^{-1}, \mathrm{GeH}_{4}^{2}$ flow rates of $200-520 \mathrm{~cm}^{3} \mathrm{~min}^{-1}$ and using both (100) and (113) oriented Ge substrates. Table I details the substrates and deposition conditions. All the epilayers were grown using the heating schedule shown in Figure 9, although the temperature and time 1. $7: 2: 1$ solution of red fuming $\mathrm{HNO}_{3}: \mathrm{HF}_{2}: \mathrm{HNO}_{3}$.

2. The $\mathrm{GeH}_{4}$ was two percent in $\mathrm{N}_{2}$ for all the depositions done in the vertical CVD apparatus. 

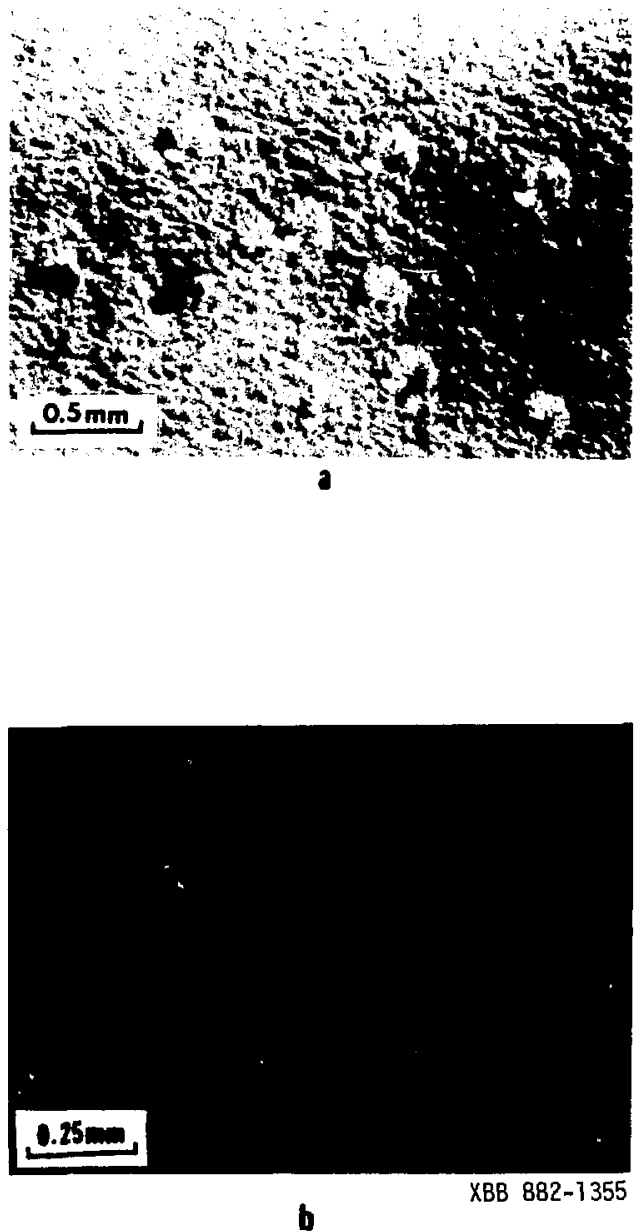

Figure 8. Optical micrographs of epilayers grown in the vertical cvo chamber. Both epilayers were grown under the same conditions $\left(2.751 \mathrm{~min}^{-1}\right.$ $\mathrm{H}_{2}, 400 \mathrm{~cm}^{3} \mathrm{~min}^{-1} \mathrm{GeH}_{4} / \mathrm{N}_{2}$ and $\sim 700{ }^{\circ} \mathrm{C}$ ) except that the wafer cleaning procedures were improved from sample I-7-1 shown in (a) to sample I-17-2 shown in (b). 


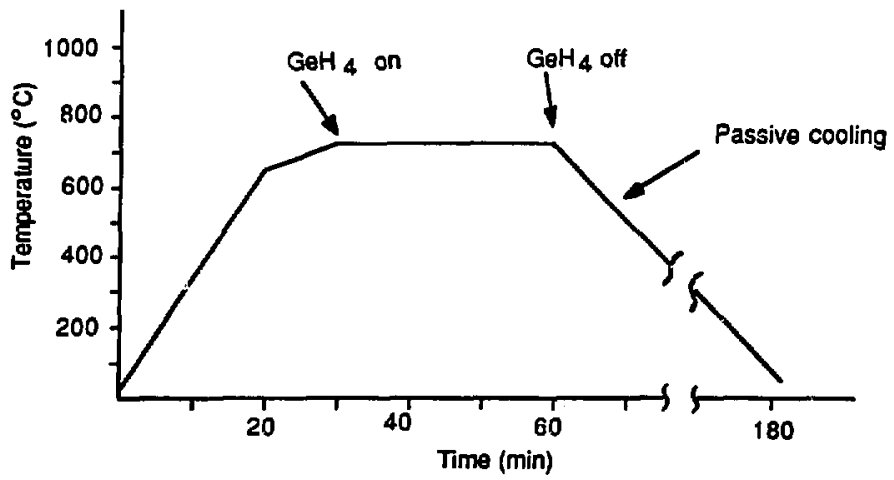

Figure 9. Heating schedule for depositions in vertical apparatus. Actual time and temperature of $\mathrm{Ge}$ deposition varied.

XBL 8841143 
during which the deposition took place varied. The initial deposition conditions were adopted from data from Papazian and Reisman (54) and Krautle, et. al. (55). When Papazian and Reisman grew Ge layers on GaAs substrates using $\mathrm{GeH}_{4}$ in $\mathrm{H}_{2}$ at atmospheric pressure, they found that the most specular layers were grown between temperatures of $650-750^{\circ} \mathrm{C}$ using 0.01-0.08 volume percent $\mathrm{GeH}_{4}$ and using total flow rates of 1.5-7 imin ${ }^{-1}$. Krautle, et. al. grew Ge on Ge and on GaAs at atmospheric pressure using $\mathrm{GeH}_{4}$ in $\mathrm{H}_{2}$ in the temperature range $500-700^{\circ} \mathrm{C}$ with a total flow rate of $1.21 \mathrm{~min}^{-1}$ and $0.01-0.1$ volume percent $\mathrm{GeH}_{4}$. The layers which were the most specular were grown at $700^{\circ} \mathrm{C}$. From these results reported in the literature, the initial depostion conditions chosen for this work were a total flaw rate of $51 \mathrm{~min}^{-1}$, growth temperatures of $700-720^{\circ} \mathrm{C}$ and 0.08 volume percent $\mathrm{GeH}_{4}$ in $\mathrm{H}_{2}$ and $\mathrm{N}_{2}$

At atmospheric pressure, and in the temperature ranges used by myse lf and the aforementioned researchers, the layer growth is limited by the mass transport of the reactant species towards the growing surface and by the desorption of hydrogen from the $\mathrm{GeH}_{x}$ radicals adsorbed on the surface $(55)$. The growth rate in the temperature regime where mass transport is rate-limiting, as opposed to the temperature regime where surface reactions are rate-limiting, is not strongly dependent on temperature, and hence it is possible to grow iniform epilayers regardless of small temperature gradients across the wafer. It was desirable that the layer growth for this work be carried out in the temperature range where mass transport is rate limiting, so that precise control over the substrate temperature would not necessary. 


\subsubsection{Electrical Characterization of the Epilayers}

The epilayers were characterized for electrical type using a thermal probe. All the epilayers, whether grown on $n$ - or p-type substrates were p-type, with the exception of the first epilayer grown. The net impurity concentration of the epilayers was determined using variable temperature Hall effect measurements $(11,91)$. The first epilayer grown, $1-1$, was n-type grown on an n-type substrate. The substrate became p-type during the deposition process by a compensating acceptor contaminant, thus the n-type film could be electrically isolated from the p-type substrate and Hall measurements could be made on the epilayer. Figure 10 shows Hall effect data for the epilayer of sample I-1-1 and I-7-1. The epilayer of I-7-1 was p-type grown on an n-type substrate. At room temperature, there were $-4 \times 10^{17} \mathrm{~cm}^{-3}$ deep acceptors and $-5 \times 10^{16} \mathrm{~cm}^{-3}$ shallow acceptors in the first epilayer grown (sample $[-1-1$ ). By the time the seventh epilayer had been grown, the cleaning procedures and/or the cleanliness of the CVD chamber itself had improved such that the net shallow impurity concentration was reduced to $-2 \times 10^{15} \mathrm{~cm}^{-3}$, as shown in the Hall data for sample 1-7-1. The deep acceptor concentration remained approximately the same. These deep acceptors were presumed to be structur:l defects, as these are p-type in Ge and no known deep elemental level in Ge has a solid solubility in $\mathrm{Ge}$ any where near those concentrations. Hall effect measurements on subsequent epilayers revealed that the net shallow impurity concentration did not decrease significantly, as shown in Figure 11, which compares an epilayer from the seventh deposition to an epilayer from the thirteenth deposition. The Hall data on the epilayer from the thirteenth deposition was typical of the later 


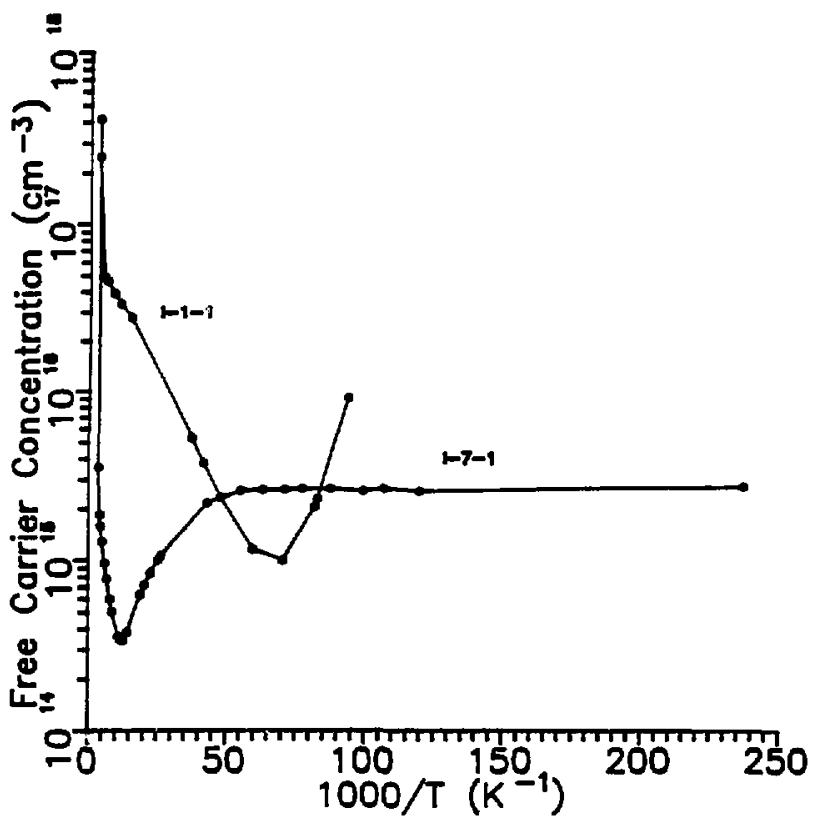

XBL 8841144

Figure 10. Hall effect data on samples $1-1-1$ and $1-7-1$ showing the decrease in net shallow acceptor concentration from 1-1-1 to a later deposition, I-7-1. 


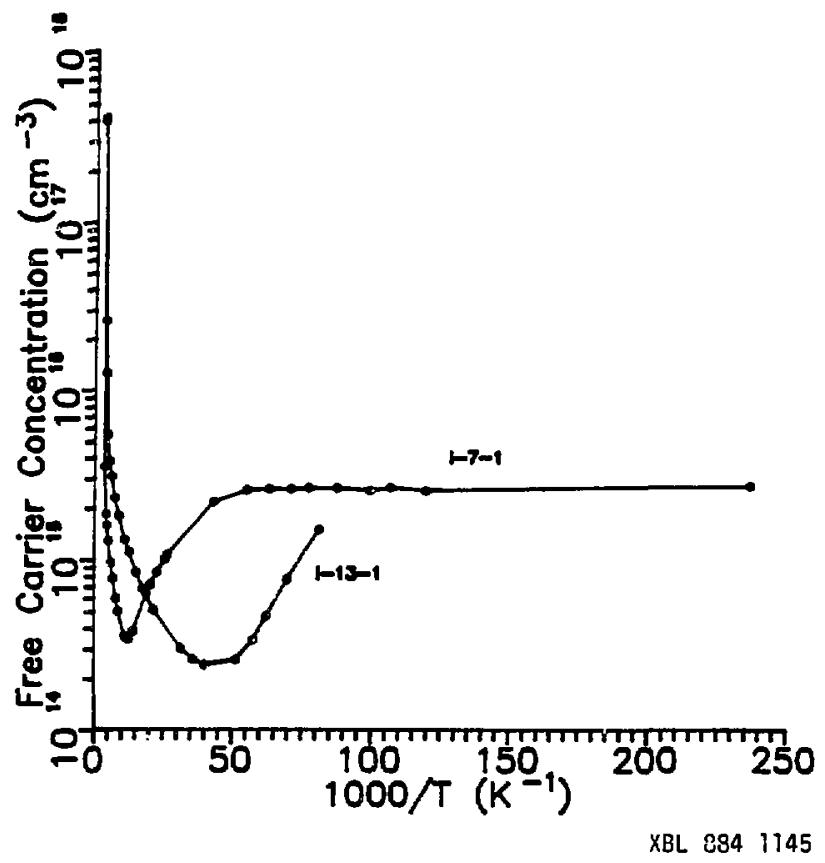

Figure 11. Hall effect data on samples $[-7-1$ and $I-13-1$ showing similar net shallow acceptor concentrations in the epilayers. 
depositions. Although the net shallow impurity concentration did not decrease significantly, the mobility in the epilayer of sample 1-13-1 was much higher at low temperature than that of $1-7-1$, as shown in Figure 12, indicating the the compensation level was lower in the later grown epilayers. However, the shallow impurity concentration in even the cleanest epilayers was so high that hopping conduction occurred and the impurity levels did not freeze out with decreasing temperature. These layers would be much too dirty to serve as blocking layers on BIB detectors -carriers from the impurity band in the heayily-doped IR-active substrate would not be blocked by these layers, because hopping conduction could obviously occur in these layers.

The predominant shallow acceptor impurity in the p-type epilayers was presumed to be boron, since this as a very common shallow acceptor contaminant in Ge. This was confirmed by Secondary I on Mass Spectroscopy (SIMS) measurements done on the epilayer of sample I-14, as shown in Figure 13. The flat profile at a concentration of $10^{15} \mathrm{~cm}^{-3}$ does not represent the true $B$ concentration but it indicates background ion counts which are the detection limits of the SIMS instrument. The boron concentration profile shown in this figure was taken to be approximate, as it was calculated relative to a known B-implant concentration in Ge. Although no systematic SIMS studies were done on these layers, the measurements definitely reveal a high boron concentration in the epilayer, on the order of $10^{15}-10^{16} \mathrm{~cm}^{-3}$.

In addition to the boron impurity in the epilayers, it was also clear that there must be copper on the order of $5 \times 10^{14} \mathrm{~cm}^{-3}$. This was known from Hall measurements of n-type substrate material which always turned p-type during the epitaxy process, if the donor concentration in 


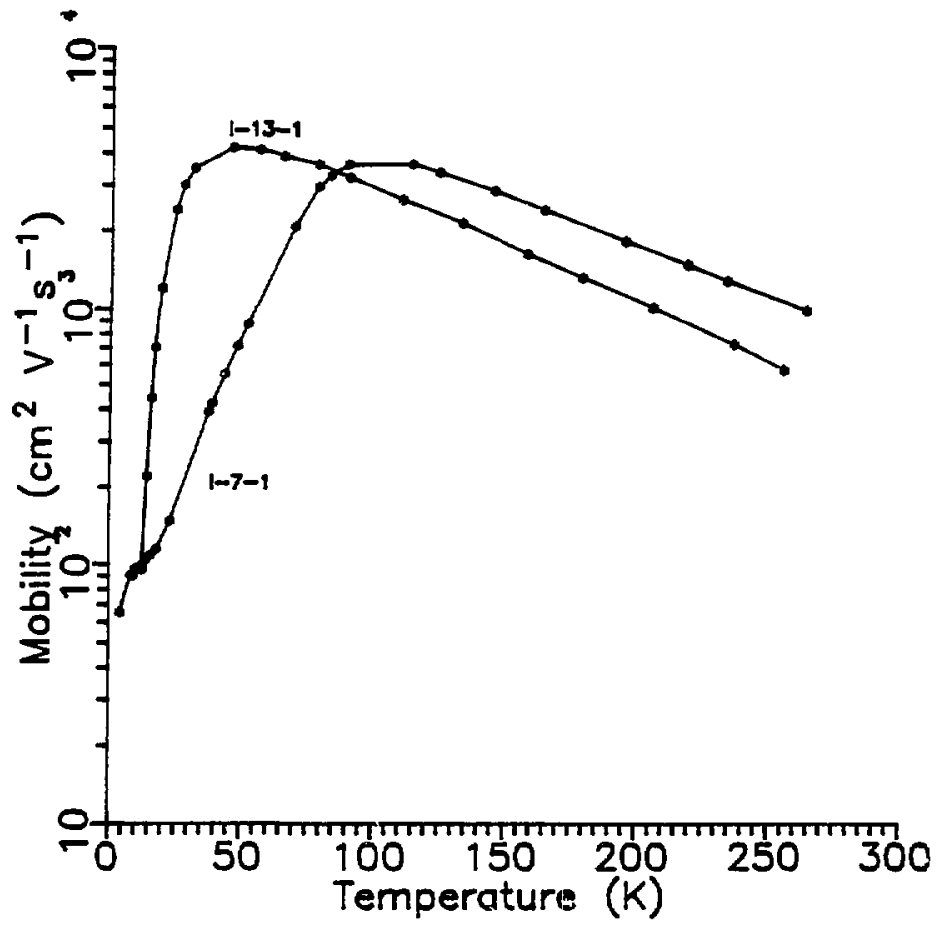

XBL 8841146

Figure 12. Mobility as a function of temperature for samples $I-7-1$ and $1-13-1$ showing the higher mobility of sample $I-13-1$ at low temperature compared with sample $1-7-1$. 


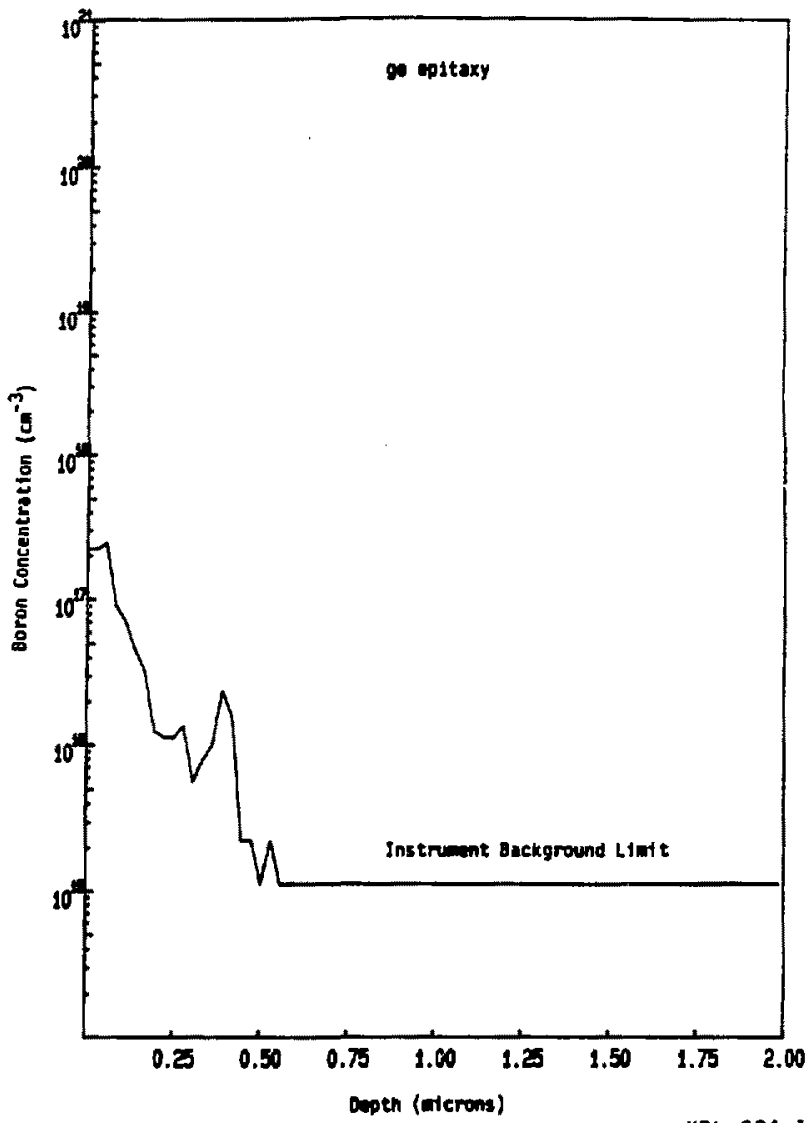

XBL $884 \quad 1147$

Figure 13. Secondary I on Mass Spectroscopy data on sample I-14 showing the $B$ concentration as a function of depth into the epilayer. $1015 \mathrm{~cm}^{3}$ is the linit of detection of $B$ in $\mathrm{Ge}$ for this ins trunent. 
the substrate was not greater than $8 \times 10^{14} \mathrm{~cm}^{-3}$. The Hall data shown in Figure 14, taken from n-type substrate material which turned p-type during the tenth epitaxy, shows that the net acceptor concentration was $-7 \times 10^{14} \mathrm{~cm}^{-3}$. The ionization energy of the acceptor can be calculated from the slope of the freezeout curve at low temperature and was $-0.044 \mathrm{eV}$, which is the first ionization energy of $\mathrm{Cu}$ in Ge. The identification of the acceptor impurity was confirmed by Photothermal I anization Spectroscopy (PTIS) performed on the same sample that the Hall data of Figure 15 were taken from. The PTIS spectrum is shown in Figure 14, and reveals the $D$ and $C$ lines of $C u$ in $\mathrm{Ge}$ at $326 \mathrm{~cm}^{-1}$ and $332 \mathrm{~cm}^{-1}$, respectively. It is thought that the copper contamination came from the final $\mathrm{HNO}_{3}$ :HF etch given the Ge wafers, just prior to insertion into the CVD apparatus, because it was not dependent on whether the $\mathrm{H}_{2}$ or $\mathrm{GeH}_{4}$ was present. The $\mathrm{Cu}$ could also have came from the graphite susceptor.

\subsubsection{Structural Characterization of the Epilayers}

The epitayer thicknesses were measured by weighing the wafers before and after deposition and also using a diamond-tipped profilameter. The weighing method was accurate to about $\pm 1 \mu \mathrm{m}$, as determined by the profiloneter measurements. The growth rate varied considerably among the depositions and was dependent on the degree to which the quartz gas aiffuser became clogged with Ge. The quartz gas diffuser height was adjusted continuously to try and avoid excess Ge deposition in the diffuser, thus no systematic growth rate measurements could be made. The growth rate varied from $0.03 \mu m \mu^{-1}$ to $0.62 \mu m m^{-1}$. 


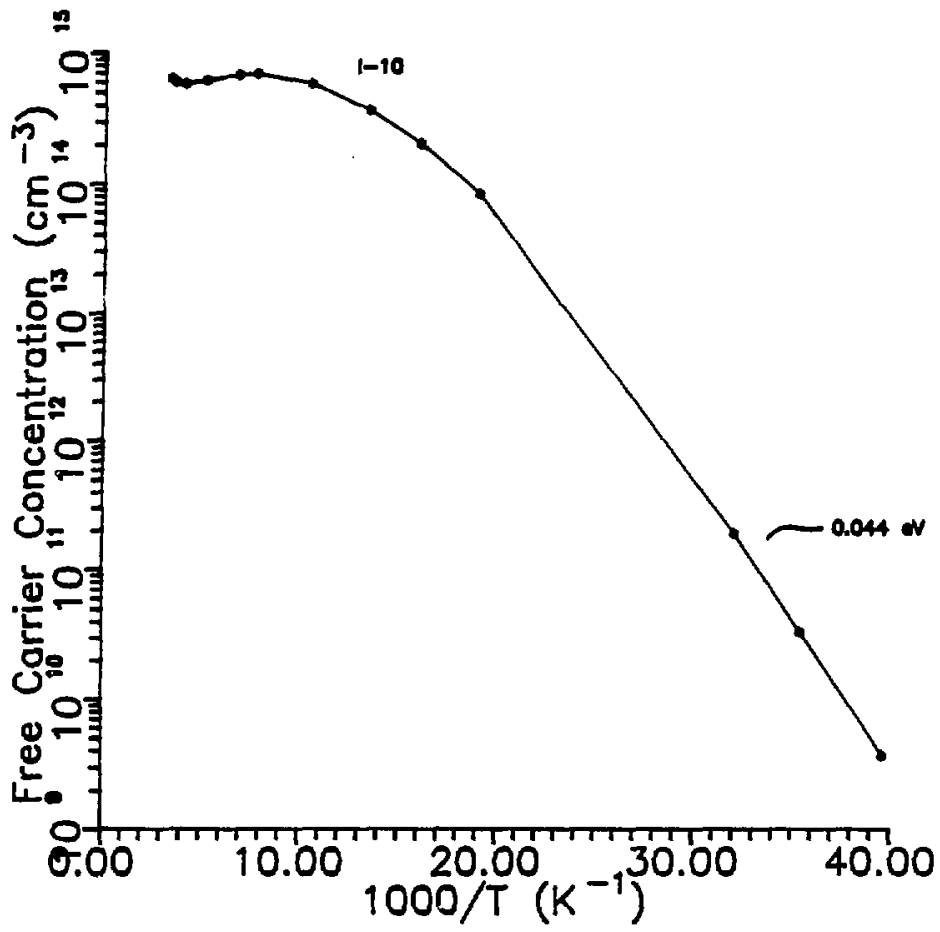

XBL $884 \quad 1148$

Fioure 14. Hall effect data on a substrate which went through the epitaxy process and became contaminated with $\mathrm{Cu}$ at a concentration of $7 \times 10^{14} \mathrm{~cm}^{3}$. The slope of the freezeout curve at low temperature is $0.044 \mathrm{eV}$ which is the first ionization energy of $\mathrm{Cu}$ in $\mathrm{Ge}$. 


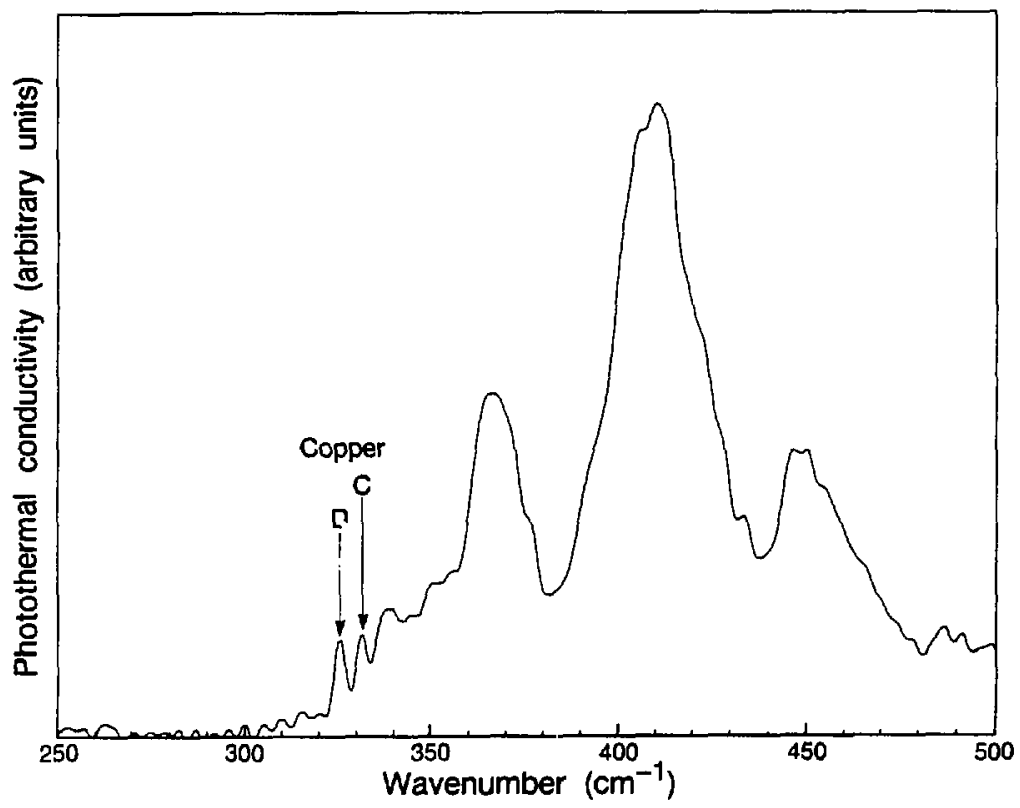

$\times$ BL 884.9605

Figure 15. Photothermal ionization spectrum of the same substrate sample shown previously in Figure 13, showing the 0 ana $C$ lines of $\mathrm{Cu}$ in $\mathrm{Ge}$ at $326 \mathrm{~cm}^{-1}$ and $332 \mathrm{~cm}^{-1}$, respectively. 
The majority of the structural characterization of the epilayers was done by visual examination using an optical microscope with confirmation using Rutherford Backscattering Spectroscopy (RBS). It is known that highly specular layers are indicative of monocrystalline layers, although to identify and quantify the defect density require much more sophisticated techniques than visual inspection. Amorphous layers can also be highly specular, so one needs another technique to verify the crystallinity. The presence of visible pits, bumps, hillocks and otherwise rough topography indicate layers of poor crystalline quality. The effect of substrate temperature on the quality of the epilayers is demonstrated by the topography of the epilayers shown in Figure 16. The three epilayers shown in this figure were all grown on (113) substrates, with $2.751 \mathrm{~min}^{-1}$ of $\mathrm{H}_{2}$ and $400 \mathrm{~cm}^{3} \mathrm{~min}^{-1} \mathrm{GEH}_{4} / \mathrm{N}_{2}$, but the growth temperature was $595-620^{\circ} \mathrm{C}$ for the layer in $16(\mathrm{a}), 650-670^{\circ} \mathrm{C}$ for $16(\mathrm{~b})$ and $720-725^{\circ} \mathrm{C}$ in $16(\mathrm{c})$. The epilayer grown at the highest temperature was highly specular with no visible defects other than a slightly rough surface, while the epilayers grown at the lower temperatures were very rough and contained a high density of triangular-like hillocks protruding from the surface.

Figure 17 shows the topography of epilayers grown on (113) and (100) substrates. The epilayers shown in $17(a)$ and $17(b)$ were grown during the same deposition, using both (100) and (113) substrates. At these low temperatures, $595-620^{\circ} \mathrm{C}$, both the (100) and (113) layers contain a very high density of pits (in the (100) case) and hillocks (in the (113) case). At temperatures high enough to produce specular (113) layers, $720-725^{\circ} \mathrm{C}$, the (100) layers are still very rough, as showin in Figure $17(c)$ and $17(d)$. 

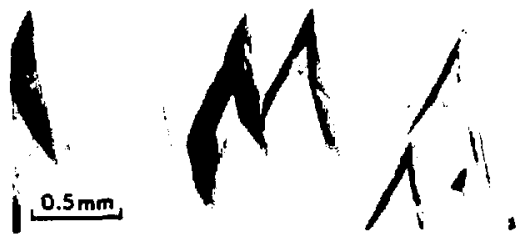

a
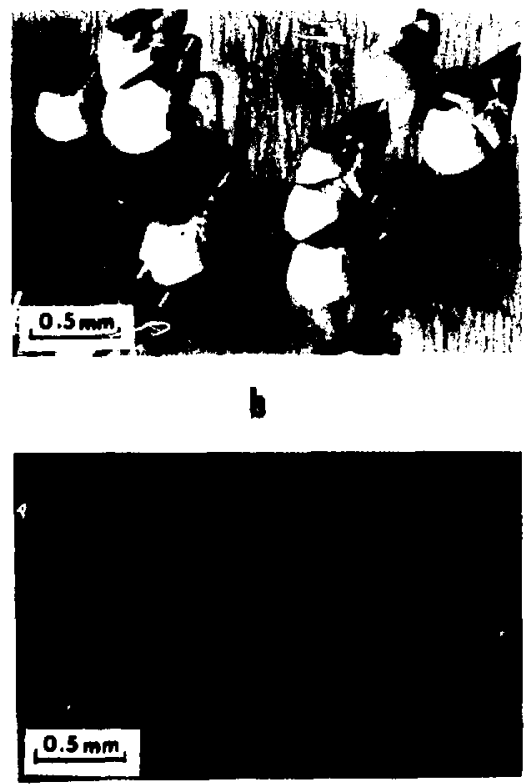

6

XBB $882-1357$

Figure 16. Optical micrographs of epilayers grown in the vertical CVD apparatus showing the effects of deposition temperature on epilayer quality. Sample I-5 shown in (a) was grown at $595-620^{\circ} \mathrm{C}, \mathrm{I}-6$ shown in (b) was grown at $650-670^{\circ} \mathrm{C}$, and I-17 shown in (c) was grown at $720-725^{\circ} \mathrm{C}$. 


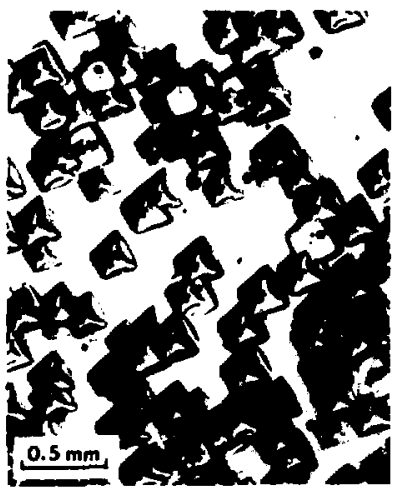

a

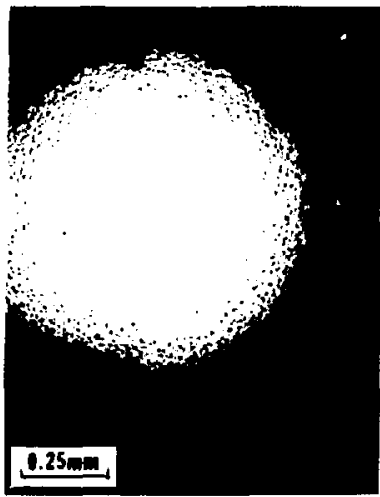

C

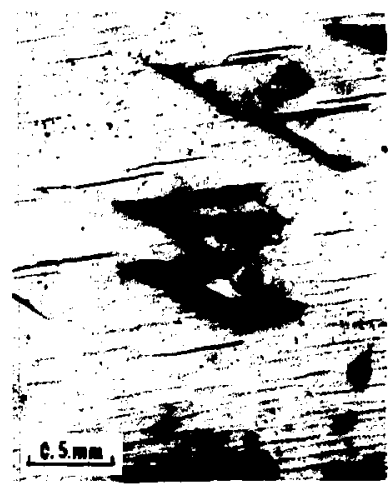

b

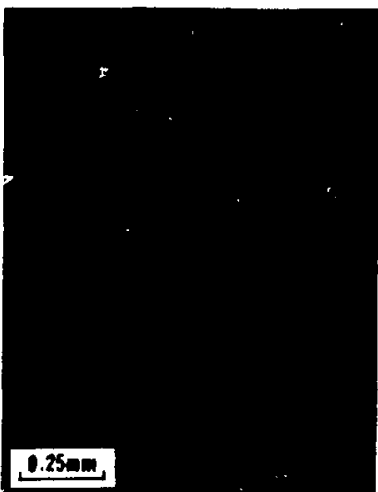

XBB 882-1356

Figure 17. Optical micrographs of epilayers grown in the vertical CVD chamber showing the effects of orientation and temperature on epitaxy quality. The epilayers shown in (a) and (b) are from sample I-5 grown at 595-6200 and are (100) and (113) orientations, respectively. The epilayers shown in (c) and (d) are from sample I-17 grown at $720-7250^{\circ} \mathrm{C}$ and are (100) and (113) orientations, respectively. 
Rutherford Backscattering Spectroscopy on the (100) layer shown previously in Figure 17(c) and on the (113) layer shown in 17(d) revealed that the (100) epilayer was heavily dislocated and most probabiy polycrystalline, while the (113) epilayer was monocrystalline. Figure 18 shows the RBS spectra for these two samples, where $18(a)$ are the spectra from the (113) epilayer grown at $720-725^{\circ} \mathrm{C}$ and $18(\mathrm{~b})$ are the spectra from the (100) layer grown under the same conditions. These samples had an area of the epilayer etched off so that the substrate could be oriented dlong a major crystallographic axis with respect to the impinging helium ion beam. Figure 18(a) shows a high He backscattering yield when the (113) substrate was randomly oriented with respect to the beam and a much lower backscattering yield when the substrate was oriented with a major axis parallel to the beam. This is the expected result, as the beam travels farther down an open crystallographic channel and less is backscattered out of the sample than when the sample is rancmly oriented with respect to the beam. When the sample was translated so that the He beam then impinged on the epilayer surface, keeping the sample in the same orientation as when channeling in the substrate occurred, the backscatter yield from the epilayer was still very low, indicating that the crystalline quility of the layer was fairly good. This technique is sensitive to approximately one percent lattice distortion and hence the dislocation density could be quite high and still not be detectable with this technique. Nevertheless, it can be saic that the (113) epilayers, grown at $720-725^{\circ} \mathrm{C}$, were probably monocrystalline. In comparison, Figure $18(b)$ shows the RBS spectra from an epilayer grown at the same time as that shown in 18(a), except on a (100) substrate. In this case, when the sample was translated from the channelled substrate position so 

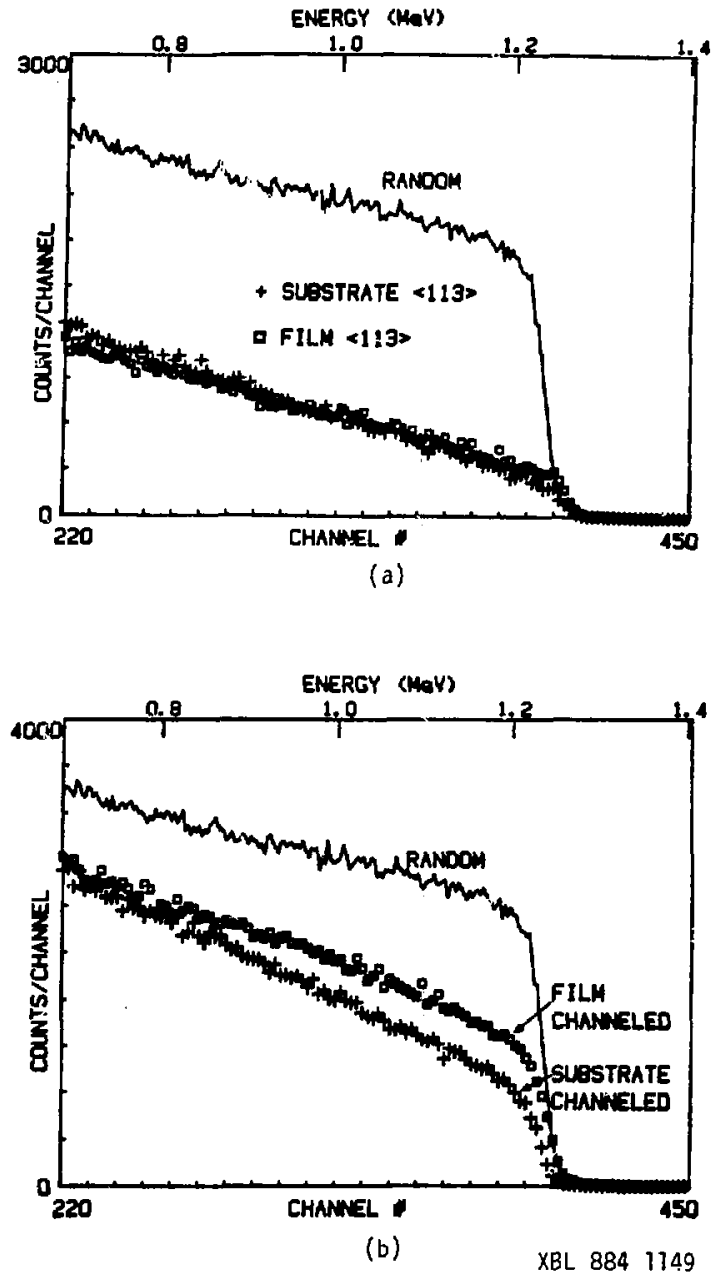

Figure 18. Rutherford backscattering spectra of: (a) a (100) epilayer grown at $720-725^{\circ} \mathrm{C}$ and (b) a (113) epilayer grown at the same time. 
chat the beam then impinged on the epilayer, the backscattered yield increased. This indicates that the crystalline quality of the epilayer is much worse than that of the (113) layer. These layers are most probably polycrystalline to some degree, but definitely not amorphous because sone beam channelling did occur.

\subsubsection{Sumary of Epitaxy using the Vertical cVo Chamber}

The best epilayers grown using the vertical CVD apparatus at atmospheric pressure contained $-2 \times 10^{15} \mathrm{~cm}^{-3}$ net shallow acceptors and $\sim 10^{17} \mathrm{~cm}^{-3}$ deep acceptors. The major shallow acceptor was boron, and the deep acceptors were most probably structural defects, because dislocations are p-type and no known deep elemental acceptors have such a high solid solubility in $\mathrm{Ge}$. These layers did not meet the $<10^{14} \mathrm{~cm}^{-3}$ shallow impurity criterion for BIB detectors. Hopping conduction could occur in these layers, as seen in the Hall effect measurements, and thus these layers could not serve as blocking layers in BIB detectors. The most specular, and thus having the highest crystalline quality, layers were grown on (113) substrates at $720-725^{\circ} \mathrm{C}$ with $2.75 \mathrm{lmin}^{-1} \mathrm{H}_{2}$ and $400 \mathrm{~cm}^{3} \mathrm{~min}^{-1}$ two percent $\mathrm{GeH}_{4}$ in $\mathrm{N}_{2}$. Temperatures higher than $725^{\circ} \mathrm{C}$ were not explored. The substrates and the epilayers became contaminated with $\mathrm{Cu}$ at a concentration of $-7 \times 10^{14} \mathrm{~cm}^{-3}$ during the deposition process. The inability to produce pure epilayers, regardless of the precautions taken during cleaning of the wafers, made it apparent that a new CVD apparatus must be built with greater attention paid to the materials used and the cleanliness of all the components during the assembly of the apparatus. In addition, a new source of pure $\mathrm{GeH}_{4}$ was 
sought. The horizontal CVD chamber, built with the utmost attention to purity and cleanliness, is described in the following section.

2.2.2 Epitaxy using Horizontal CVD at Atmospheric Pressure

\subsubsection{Apparatus and Epitaxy Procedure}

A schematic of the horizontal CVD apparatus is shown in Figure 19, while Figure 20 is a photograph of the actual apparatus. In contrast to the vertical chamber, the horizontal chamber was designed so that the gas flow would be laminar and unobstructed through the quartz tube. All the stainless steel tubing used to transport gas was cleaned in TCA, etched with $\mathrm{HF}$ and electropolished in a $\mathrm{HNO}_{3}$ solution after which it was kept wrapped in plastic until assembly with glove-protected hands. All the stainless tubing was connected to the various components with high vacuum fittings ${ }^{2}$ and high vacuum valves ${ }^{2}$. There were several valves in the gas lines to enable the pump out of air prior to introduction of the CVD gases.

As with the vertical chamber, the hydrogen was purified ${ }^{3}$ prior to entering the chamber. In addition, the inert gas (helium instead of nitrogen) was purified with a chemical resin gas purification system ${ }^{4}$.

1. VCR metal fittings from Cajon Co., Solon, Ohio.

2. Nupro stainless steel vacuum valves from Nupro Co., Willoughby, Ohio.

3. Model HP-10 hydrogen purifier from Johnson-Mathey, West Chester, Pennsylvannia.

4. Nanochem gas purifier L-50t from Semi-Gas Systems, Inc., San Jose California. 


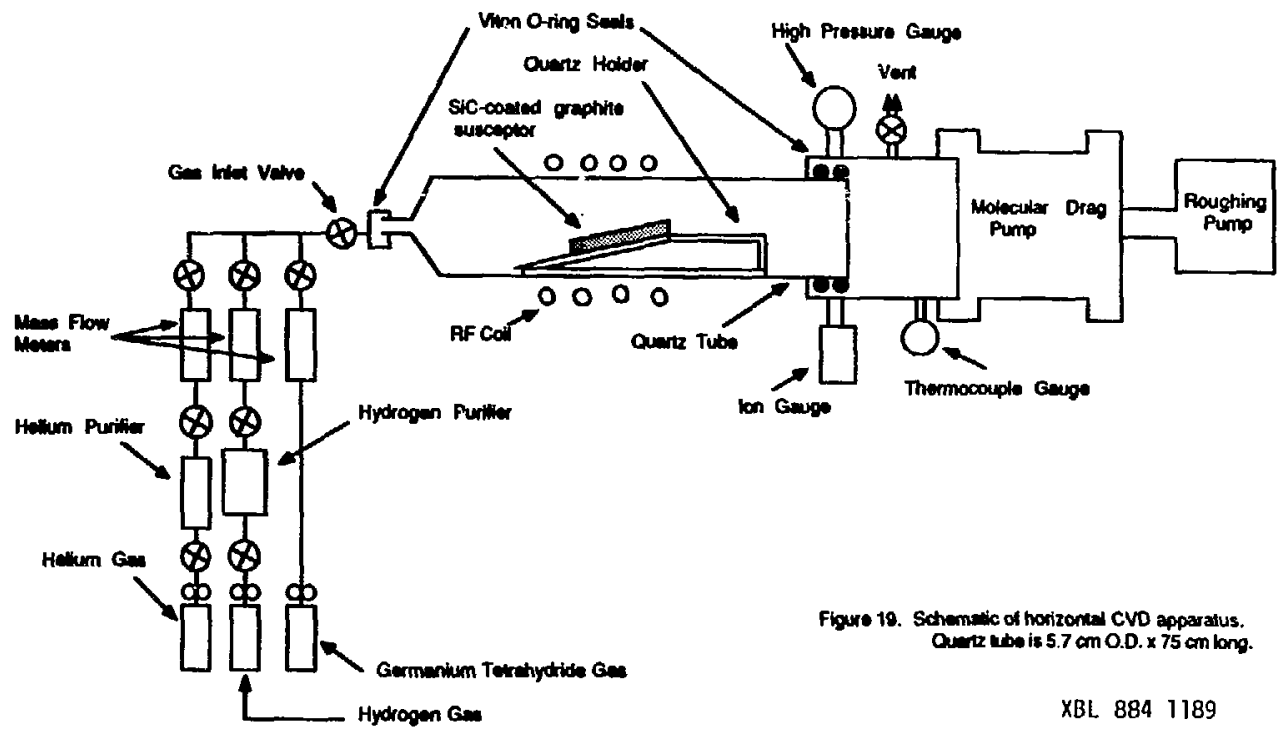




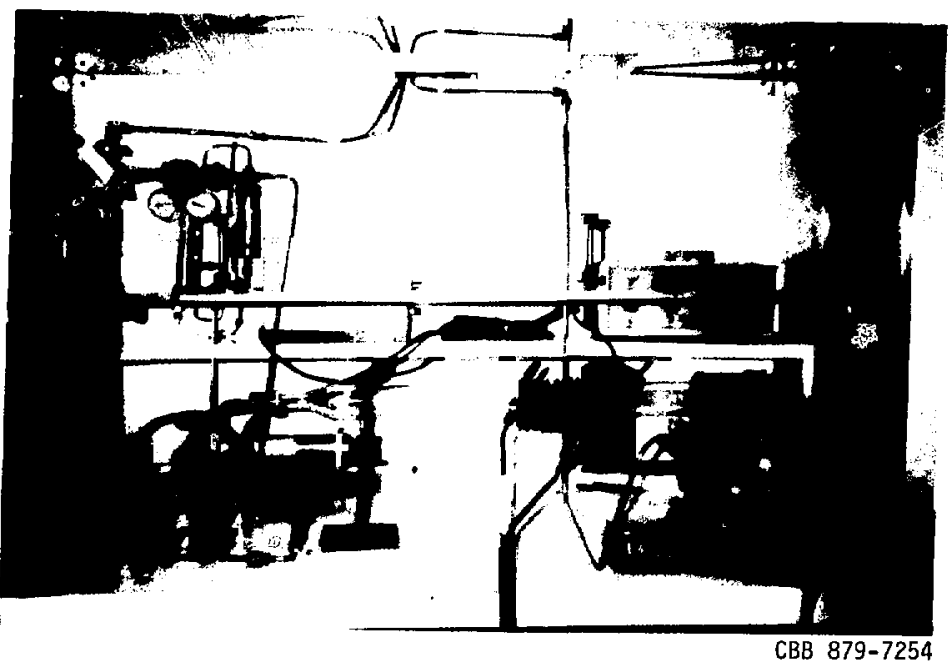

Figure 20. Photograph of the horizontal CVD chamber. 
The $\mathrm{GeH}_{4}$ was pure ${ }^{1}$ and not diluted with $\mathrm{N}_{2}$ as previously. A crasspurge system ${ }^{2}$ was attached to the $\mathrm{GeH}_{4}$ cylinder to prevent air from entering the gas lines when the cylinder was changed, but no additional purification was done on the $\mathrm{GeH}_{4}$ gas. The gas flow rates were controlled and monitored by mass flow meters ${ }^{3}$. The quartz tube which comprised the reaction chamber itse?f was etched with HF, rinsed in distilled methanol and baked in-situ prior to use. The susceptor was ultrapure graphite coated with $\mathrm{SiC}^{4}$ and baked in situ at $800^{\circ} \mathrm{C}$ for one hour prior to use.

The Ge substrates were polished as described previousiy in Section 2.2.1.1. They were cut $\sim 1-3^{\circ}$ off axis, as previously, and the dislocation density prior to epitaxy was $\sim 10^{3} \mathrm{~cm}^{-2}$. The doping impurities and concentrations of the substrates are described in Table II. The two substrates on which layers were grown and out of which BIB detectors were fabricated are included in Table II. Substrate number 582 was used in deposition number 11-13 and had a Ga concentration of $5 \times 10^{15} \mathrm{~cm}^{-3}$ and a resicial donor concentration of $\sim 10^{10} \mathrm{~cm}^{-3}$. This doping level is not high enough to measureably reduce the Ga ionization energy, but it is high enough that some impurity banding and hopping occur. The Hall datá of Figure 21, taken from a lower doped region of the same 582 crystal with a doping level of $-8 \times 10^{14} \mathrm{~cm}^{-3}$, shows the hopping conduction

1. GeH 4 gas from Mitsui-Toatsu Chemicals, Inc., Tokyo, Japan. Gas analysis provided by the vendor: $32 \mathrm{ppm} \mathrm{H}_{2}, 1.8 \mathrm{ppm} \mathrm{N}_{2}, 0.1 \mathrm{ppm}$ hydrocarbons, $0.2 \mathrm{ppm}$ chlorides.

2. Model 4774 cross-purge assembly from Matheson Gas Products, East Rutherford New Jersey.

3. Model FC-280 mass flow meters and model R0-28 control unit from Tylan Corp., Carson, California.

4. SiC-coated graphite susceptor from Stackpole-Ultracarbon Corp., Bay City, Michigan. 
Table II

Sample Substrate $\mathrm{H}_{2} \quad \mathrm{GeH}_{4}$ Growth Growth Film Vol.\% Average No. conc. $\mathrm{cm}^{-3} 1 \mathrm{~min}^{-1} \mathrm{cni}^{3} \mathrm{~min}^{-1}$ temp. ${ }^{\circ} \mathrm{C}$ time, frin type GeH 4 thick., um

\begin{tabular}{|c|c|c|c|c|c|c|c|}
\hline II -6 & $\begin{array}{l}\text { Ge:P }(113) \\
5 \times 10^{13}\end{array}$ & 0.85 & 2 & 700 & 110 & p & 0.23 \\
\hline II -7 & $\begin{array}{l}\mathrm{Ge}: \mathrm{Ga}_{5}(113) \\
5 \times 10^{15}\end{array}$ & 0.85 & 2 & 700 & 180 & $p$ & 0.23 \\
\hline II -8 & $\begin{array}{l}\text { Ge:Sb }(100) \\
8 \times 10^{14} \\
\text { Ge:A1 (113) }\end{array}$ & 0.85 & 2 & 700 & 165 & $p$ & $\begin{array}{l}0.23 \\
0.23\end{array}$ \\
\hline II -9 & $\begin{array}{l}\text { Ge:Sb (100) } \\
8 \times 10^{14} \\
\text { Ge:Al (113) } \\
1 \times 10^{11}\end{array}$ & 0.85 & 2 & 700 & 120 & $p$ & 0.23 \\
\hline
\end{tabular}

\begin{tabular}{|c|c|c|c|c|c|c|c|}
\hline II-11 & $\begin{array}{l}\text { Ge:Sb }(100) \\
8 \times 10^{14} \\
\text { Ge:Ga (113) }\end{array}$ & 0.85 & 2 & 735 & 510 & $\begin{array}{l}p \\
p\end{array}$ & 0.23 \\
\hline
\end{tabular}

\begin{tabular}{|c|c|c|c|c|c|c|c|}
\hline II -12 & $\begin{array}{l}\text { Ge:P } \\
2 \times 10^{14}\end{array}$ & 0.85 & 2 & 735 & 120 & $p$ & 0.23 \\
\hline $11-13$ & $\begin{array}{l}\text { Ge:Ga (113) } \\
5 \times 10^{15} \\
\text { Ge:P }(113) \\
6 \times 1013\end{array}$ & 0.85 & 2 & 735 & 430 & $\begin{array}{l}p \\
p\end{array}$ & 0.23 \\
\hline
\end{tabular}

\begin{tabular}{|c|c|c|c|c|c|c|c|}
\hline I I-14 & $\begin{array}{l}\text { Ge:P }(113) \\
2 \times 1014 \\
\text { Ge:Ga (113) } \\
2 \times 1016\end{array}$ & 0.85 & 2 & 735 & 420 & $p$ & 0.23 \\
\hline $11-15$ & $\begin{array}{l}\text { Ge:P (113) } \\
2 \times 1014\end{array}$ & 0.85 & 2 & 735 & 240 & $p$ & 0.23 \\
\hline II-16 & $\begin{array}{l}\text { Ge:P }(113) \\
2 \times 10^{14} \\
\text { Ge:Ga }(113) \\
2 \times 10^{16}\end{array}$ & 0.85 & 2 & 735 & 240 & $\begin{array}{l}p \\
p\end{array}$ & 0.23 \\
\hline
\end{tabular}




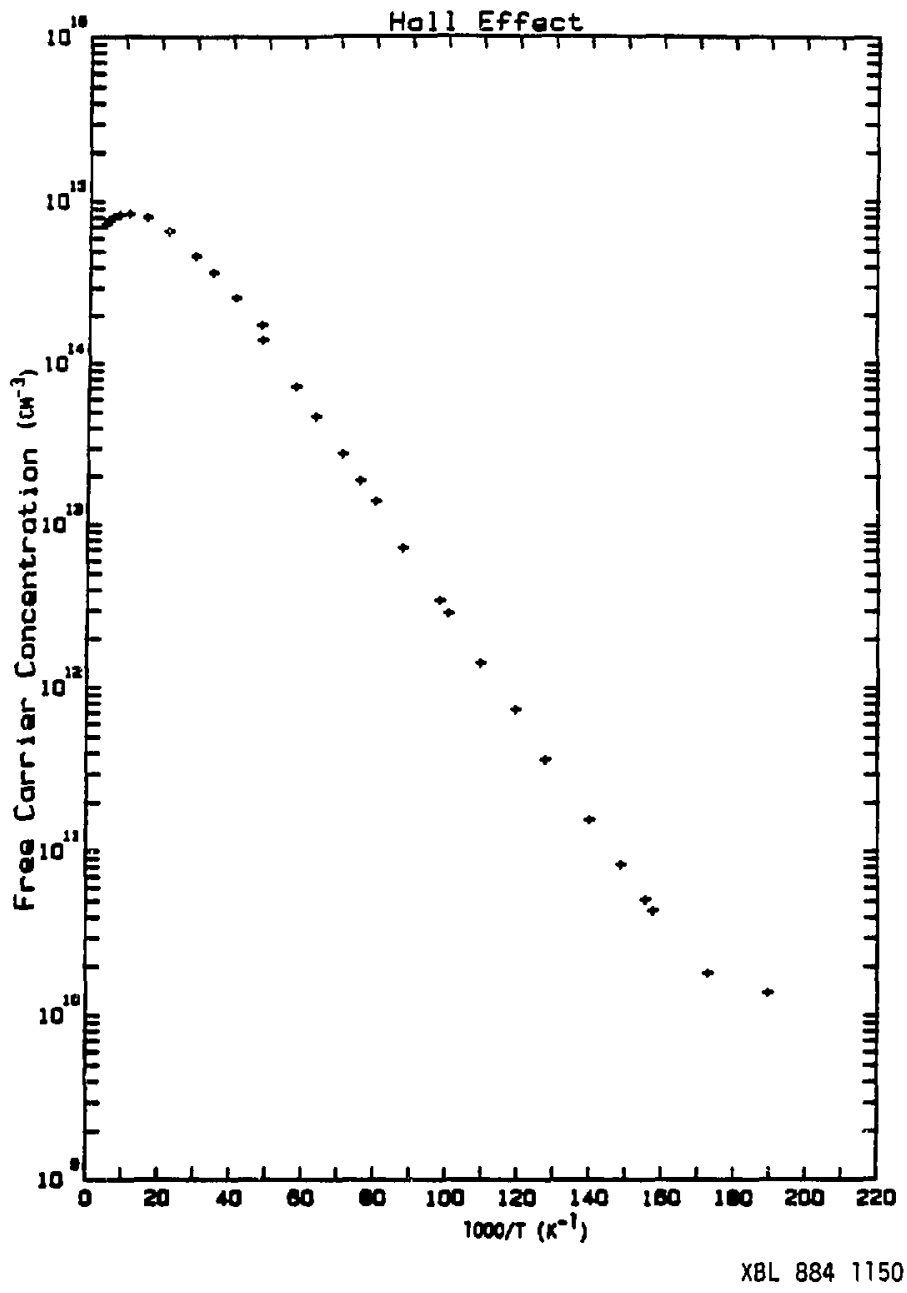

Figure 21. Hall effect data on crystal number 582-5, which is part of the same boule that the substrates in deposition II-13 were from. The net Ga concentration is $-8 \times 1014 \mathrm{col} 3$ for the sample shown here, whereas it was $5 \times 1015 \mathrm{~cm}^{3}$ in the material used for the epitaxial depositions. 
"tail" which occurs at $-10^{10} \mathrm{~cm}^{-3}$. It is apparent that hopping conduction does occur at these doping levels, al though not as much as woulo occur in more heavily doped material. The actual residual aonor concentration cannot be determined from this data, because the carrier concentration freezeout curve does not show a region of full slope freezeout $(0.011 \mathrm{eV}$ for $\mathrm{Ga}$ in $\mathrm{Ge})$, which would begin at the compensating concentration level. However, it is determined from this ada that there can only be a maximum of $10^{10} \mathrm{~cm}^{-3}$ donors in this crystal, which makes it ideal for heavily doped IR-active layers in BIB detectors.

Cystal 746 was also used as a substrate to grow epilayers which were then fabricated into BIB detectors. 746 was doped with Ga at a level of $2 \times 10^{16} \mathrm{~cm}^{-3}$, as shown in the Hall data of Figure 22 . As seen in this figure, significant impurity banding ano hopping conduction occur in this material, as the carriers oo not freeze out with decreasing temperature. The residual donor concentration cannot be determined from this oata, but was estimated to be $-5 \times 10^{11} \mathrm{~cm}^{-3}$ based on residual impurity data from crystals grown before and after crystal 746 in the same crystal puller. Spreading resistance data, shown in Figure 23 , reveal that this material is uniformly doped, with no concentration striations along the axis or radius of the crystal.

The deposition conditions for the epllayers grown at atmospheric pressure in the horizontal chamber are listed in Tade 11 . All the layers were deposited under the same $\mathrm{H}_{2}$ flow rate of $0.35 \mathrm{imin}^{-1}$ ano Ger, flow rate of $2 \mathrm{~cm}^{3} \mathrm{~min}^{-1}$. The deposition temperatures were eitner $700^{\circ} \mathrm{C}$ or $735^{\circ} \mathrm{C}$, and the deposition times varied from run to $r ! n$. Increasing the substrate temperature from $760^{\circ} \mathrm{C}$ to $735^{\circ} \mathrm{C}$ resulteo in more specular (100) layers, but did not significantly affect 


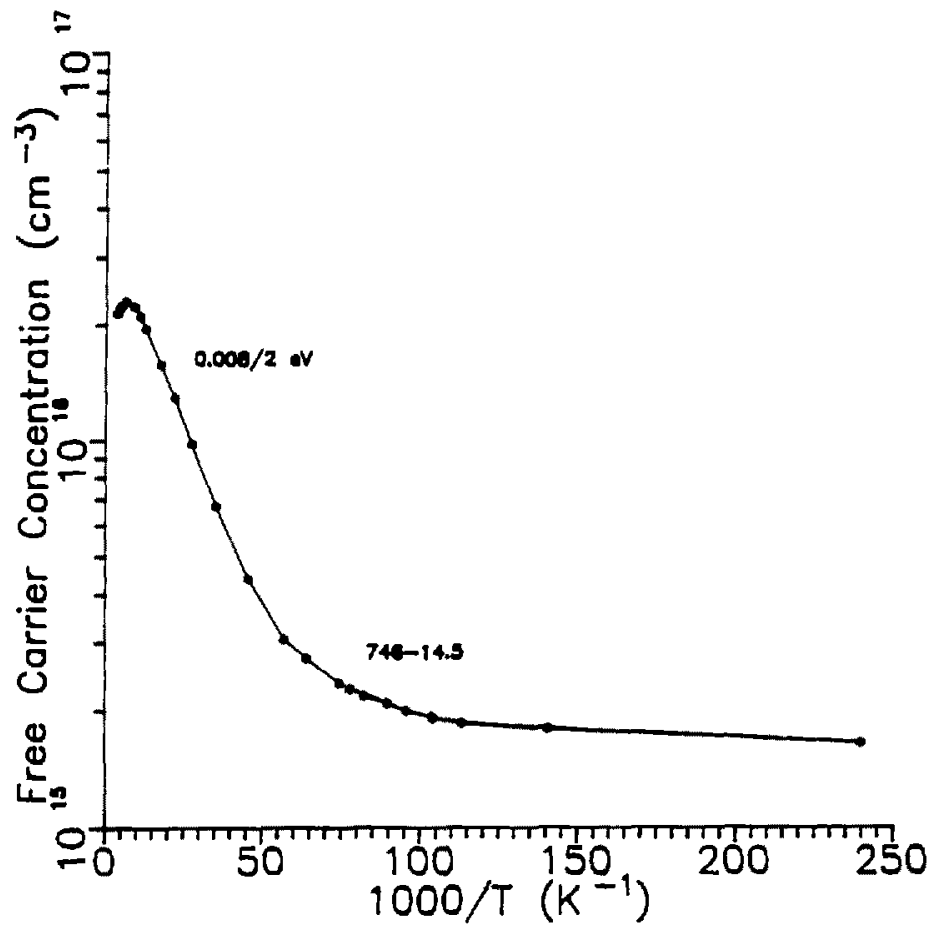

XEL $884 \quad 1751$

Figure 22. Hall effect data for crystal number 746-14, which is the sane material that depositions $I I-14$ and $I I-16$ were done on. 

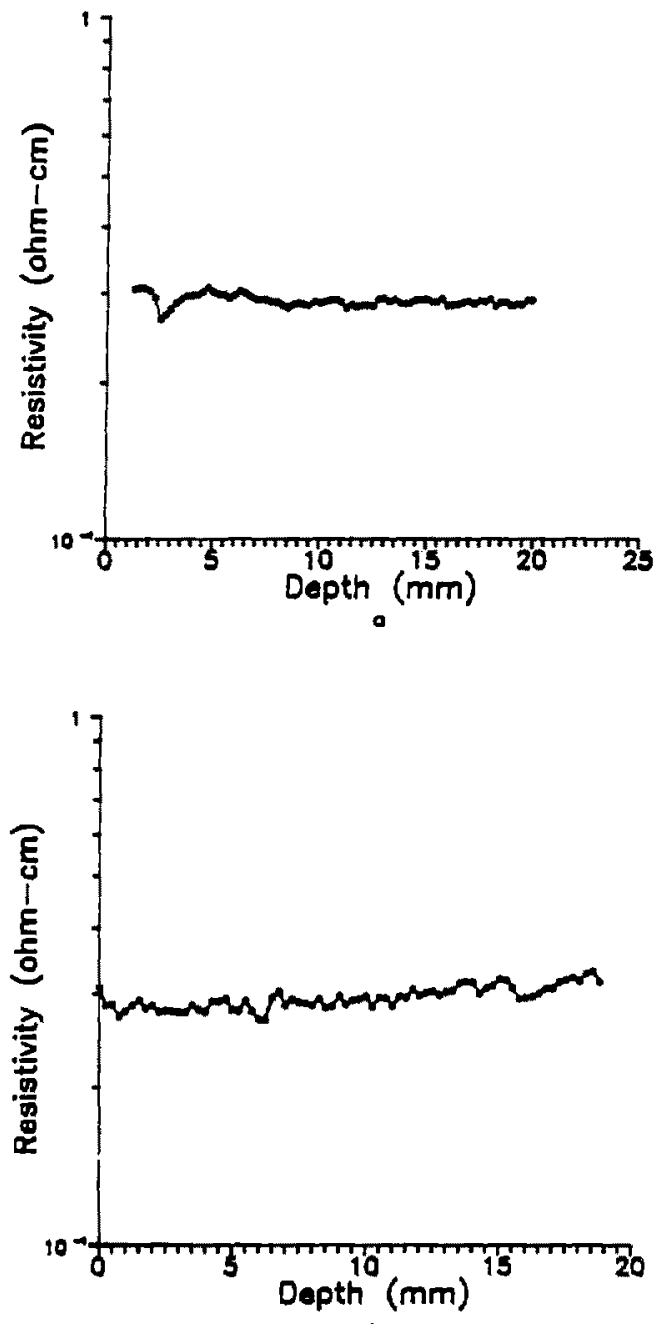

XBL $884 \quad 1152$

Fiọure 23. Spreading resistance data on crystal number 746-10, usea for cepositions $[1-14$ and $11-16$, in the (a) raoial, and (b) axial directions of the boule. 
the appearance of the (113) layers. The heating schedule for depositions 1I-6 through II-10 is shown in Figure 24(a) The heating schedule for depositions II-II through II-16 was the same, with the addition of a slow cooling sequence and an anneal at $500^{\circ} \mathrm{C}$ for one hour, as shown in Figure 24(b). Unlike depositions done in the vertical apparatus, all the depositions in the norizontal chamber included a 15-ninute bake in $\mathrm{H}_{2}$ at $800^{\circ} \mathrm{C}$, immediately prior to introducing the $\mathrm{GeH}_{4} \mathrm{gas}$, to ensure reduction of the native $\mathrm{Ge}$ oxide.

\subsubsection{Electrical Characterization of the Epilayers}

All the epilayers grown in the horizontal CVD chamber were p-type, regardless of the type of substrate they were grown on. The purity of the epilayers was determined by Hall effect measurements on p-type layers grown on $n$-type substrates. The Hall data of Figure 25 were taken from the epilayer of sample II-12 and were typical of Hall data from other epilayers grown in the horizontal chamber. From this data it can be seen that there are $-5 \times 10^{16} \mathrm{~cm}^{-3}$ deep acceptors at room temperature very similar to those seen in the layers grown in the vertical chamber. As discussed previously, these acceptors are thought to be structural defects in the epilayer and/or at the substrate/epilayer interface. There are $-3 \times 10^{13} \mathrm{~cm}^{-3}$ net acceptors at high temperature which freeze out with an energy of $0.021 \mathrm{eV}$. As this is very close to half of the first ionization of $\mathrm{Cu}$ in $\mathrm{Ge}$, it is assumed that this acceptor is $\mathrm{Cu}$ and it must not be closely compensated because the half-ionization energy slope is abserved in the Hall ada (91). At lower temperatures there are $-6 \times 10^{11} \mathrm{~cm}^{-3}$ shallow acceptors with an ionization energy of $-0.011 \mathrm{eV}$. 


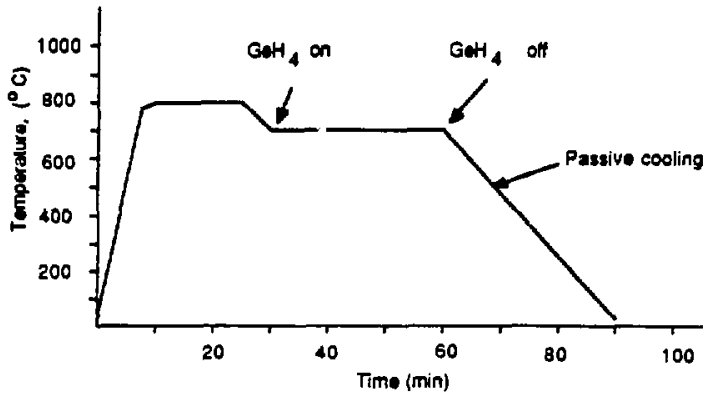

Figure 24(a). Heating schedule for depositions II-6 through 11-10. Timo and temperature of actud deposition varied.

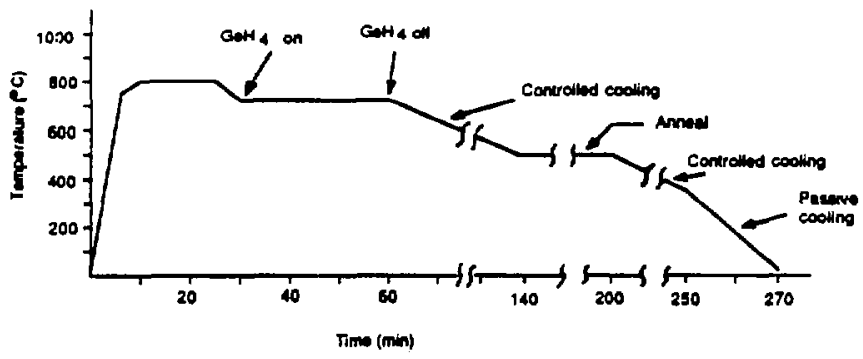

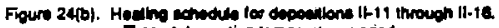
Tims in depariton kmperture veried. 


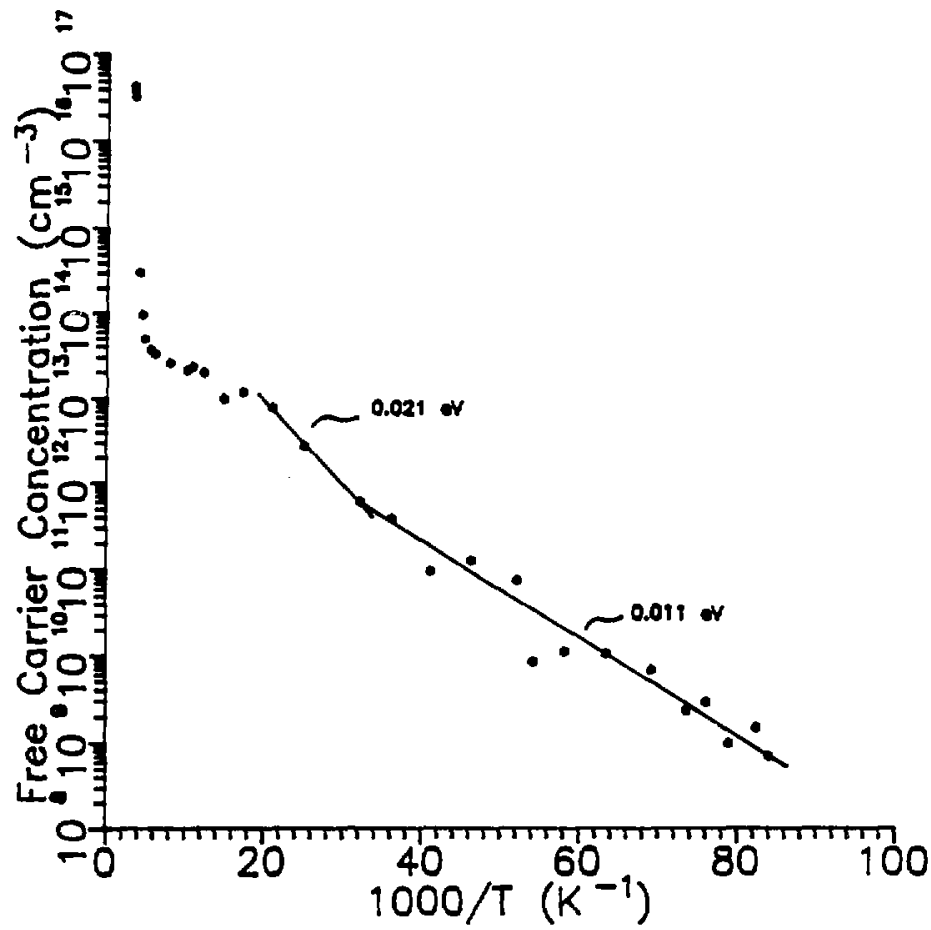

XBL $88.4 \quad 1154$

Figure 25. Hall effect data on a typical epilayer orown in the horizontal CVO chamber using the slow cooling and annealing sequence. 
Shallow acceptors, such as $B$ and $G d$, in Ge have ionization energies of this order and thus these must be very closely compensated with residual donors, since only the full ionization energy freezeout slope is observed (91). The scatter in the low temperature data is attributed to contact problems which occur when the sample resistance is very high, as it was in this case for very pure and very thin epilayers. As the hall data just discussed shows, the epilayers grown in the horizontal chamber were very pure and thus could potentially act as excellent blocking layers in BIB detectors. No hopping conduction could occur in layers of such low impurity concentration and hence these layers could effectively block hopping conduction from the heavily doped IR-active layer in a BIB device.

The substrates used for epitaxy became contaminated with Cu during the deposition process, which also occurred in the vertical cvo chamber, as discussed previously. N-type substrates doped to $8 \times 10^{14} \mathrm{~cm}^{-3}$ did not turn $p$-type during epitaxy, but n-type substrates doped to $2 \times 10^{14} \mathrm{~cm}^{-3}$ did become p-type, indicating that the compensating $\mathrm{Cu}$ concentration was somewhere between $2-8 \times 10^{14} \mathrm{~cm}^{-3}$. After a slow cooling sequence was introduced, as shown is Figure $24(b)$, the Cu concentration was reduced to $-6 \times 10^{13} \mathrm{~cm}^{-3}$. The solid solubility of $\mathrm{Cu}$ in $\mathrm{Ge}$ at $500^{\circ} \mathrm{C}$ is $<10^{14}$ $\mathrm{cm}^{-3}(92)$ and thus the slow cooling and one-hour anneal at $500^{\circ} \mathrm{C}$ was sufficient to cause precipitation of sane of the Cu. Figure 26 shows Hall effect data on two n-type substrates which vecame contaminated with Cu during the epitaxy process. The residual Cu concentration with passive cooling was $6 \times 10^{14} \mathrm{~cm}^{-3}$ and after slow cooling with the anneai at $500^{\circ} \mathrm{C}$ was $6 \times 10^{13} \mathrm{~cm}^{-3}$. The acceptor ionization energy, determined from the low temperature portion of the curve, is $0.044 \mathrm{eV}$, which identifies it as Cu. The Cu identification was not verified with 


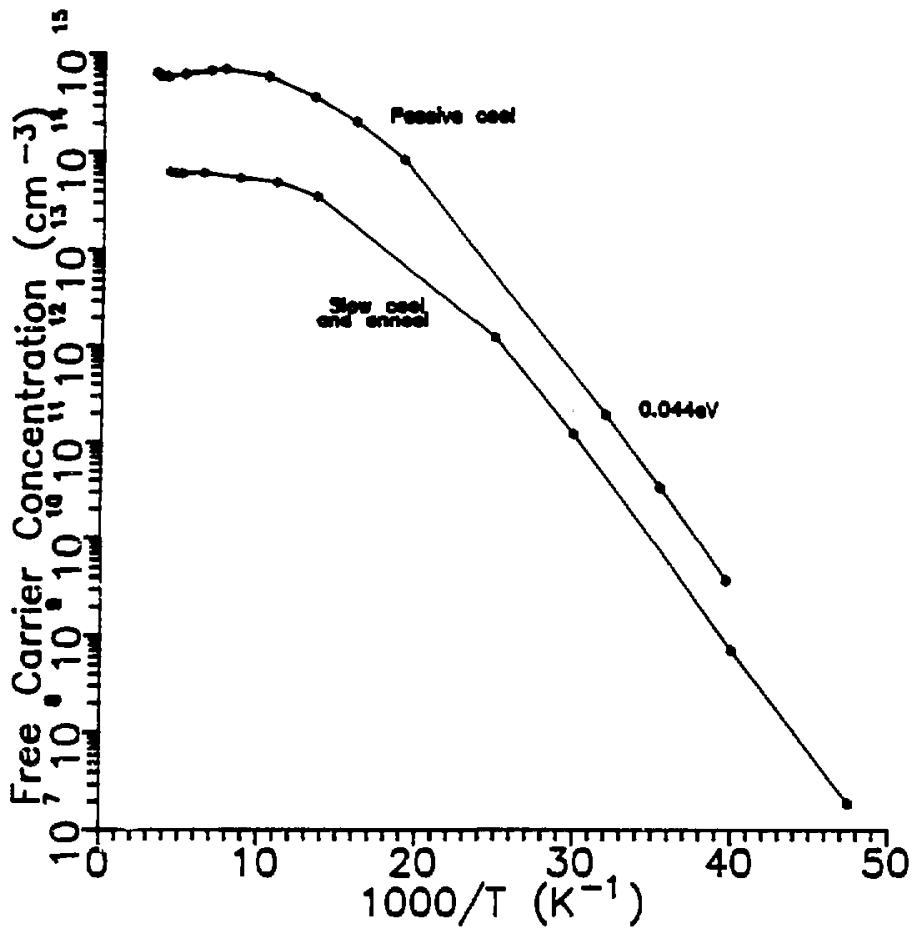

XBL 8841155

Figure 26. Hall effect data on two substrates which went through the epitaxy process using, (a) the passive cooling schedule shown in Figure 24(a) and. (b) using the controlled cooling schedule shown in Figure $24(b)$. 
other analytical techniques, as this was done previously on epilayers grown in the vertical cvo chamber.

Spreading resistance measurements, as a function of depth from the epilayer surface, were done on an area of sample II-13 and on two areas of sample II-16. The data shown in Figure 27(a) are from an area of epilayer II -13 which was closest to the leading edge of the susceptor where the growth rate was $\sim 0.06 \mu_{m i n}{ }^{-1}$. The continuous decrease in resistivity with depth from the surface is due to an increasing Ga concentration from solid state diffusion from the heavily doped Ge:Ga substrate. The very low resistivity at the surface corresponds to an implanted layer, and the near surface resistivity corresponds to an acceptor concentration of $-5 \times 10^{13} \mathrm{~cm}^{-3}$ which is the residual $\mathrm{Cu}$ concentration in the epilayer. Figure $27(b)$ shows resistivity as a function of depth from the surface for sample $11-16$. This sample also came from the leading edge of the wafer where the growth rate was $-0.06 \mu m-\mathrm{in}^{-1}$. The Ga diffused region is $-7 \mu \mathrm{m}$ thick, as was also observed in $I I-13$, and the flat resistivity profile at the surface corresponds to the residual cu concentration in the epilayer. Figure $27(\mathrm{c})$ was taken from II-16 from an area farthest from the leading edge where the growth rate was $-0.02 \mu \mathrm{mmin}^{-1}$, and thus the layer is thinner than that shown in $26(\mathrm{~b})$. The variation in the growth rate across the length of the wafer is discussed in detail in the following section.

\subsubsection{Structural Characterization of the Epilayers}

The epilayer thicknesses were determined by weighing the substrate wafers before and after deposition. The growth rate varied considerably 


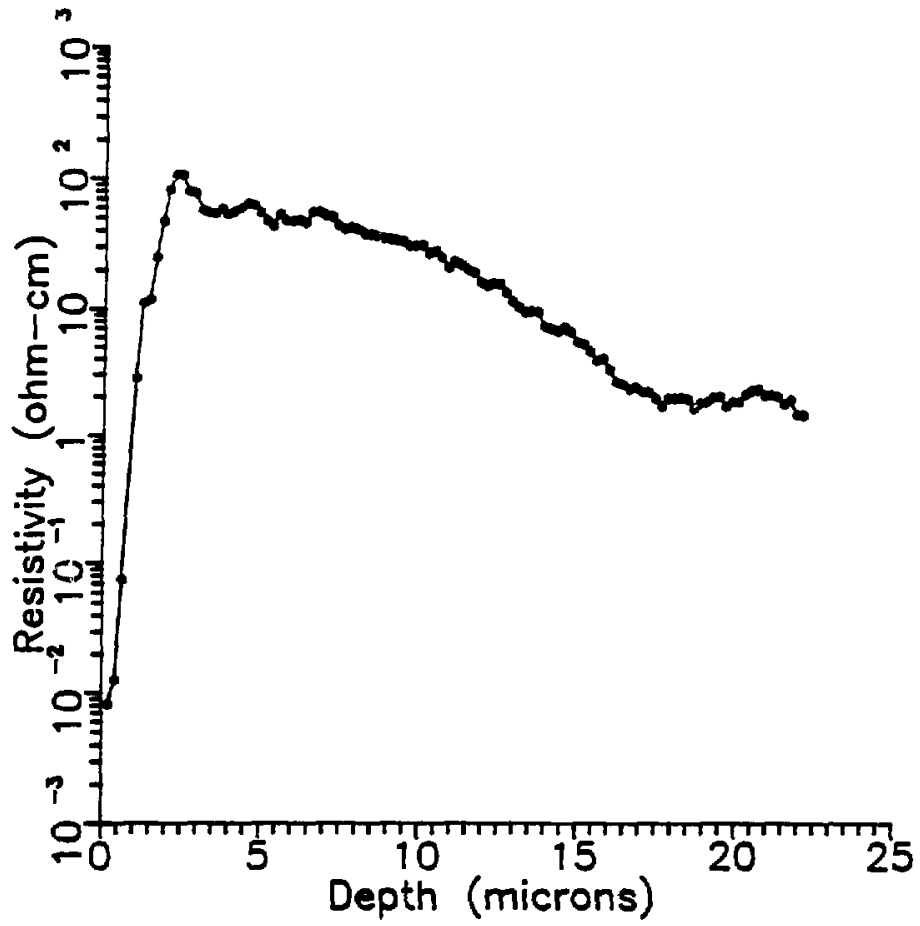

XBL 8841156

Figure $27(a)$. Spreading resistance as a function of cepth into the epilayer for an area of $I I-13$ close to the leading eage of the wafer where the growth rate was $-0.06 \mu m m^{-1}$. The very low resistivity at the surface is due to a B-implanted layer. The epilayer is -15 um thick. 

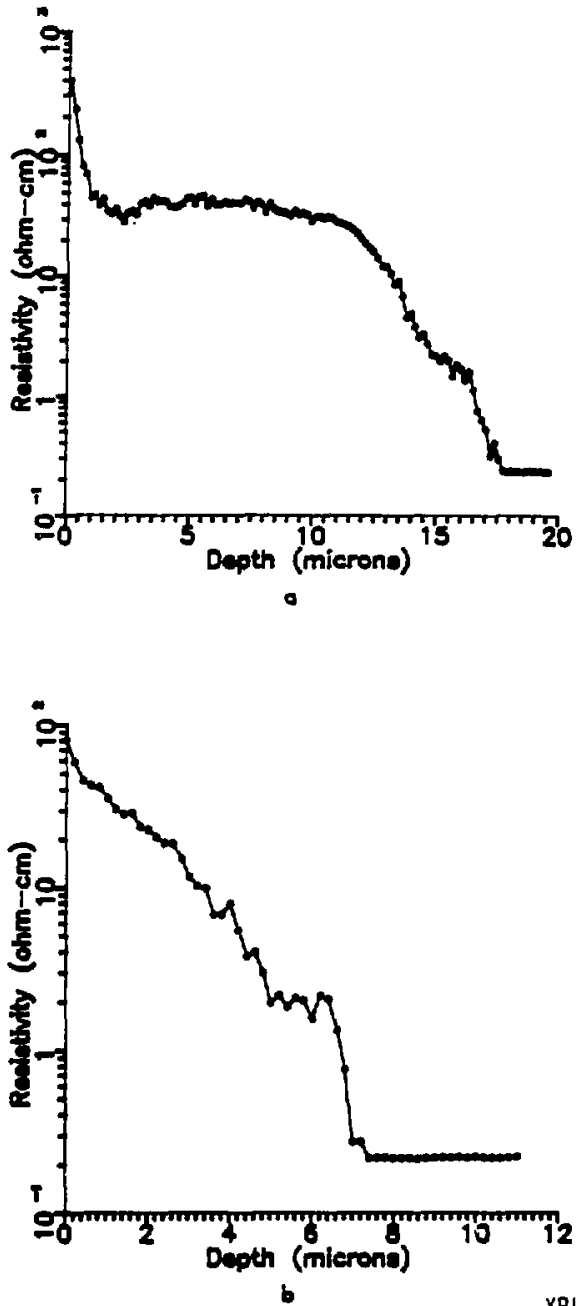

Figure $27(b)$ and (c). Spreading resistance as a function of depth from the epilayer surface for: (b) an area of epilayer close to the leading edge of the wafer in $I I-16$ where the growth rate was -0.06 umini-1, ano (b) an area of epilayer farthest from the leading eoge of the same wafer where the growth rate was -0.02 ummin $^{-1}$. The slight rise in resistivity at the very surface is due to the native oxide. 
across the length of the wafer, as is shown in the schematic of ligure 28. This phenomenon has been reported in the literature $(74,80,93)$ ano is partially due to the so-called "stagnant gas boundary layer" which develops over the hot wafer during atmospheric CVD when the reactor configuration is similar to the horizontal configuration used here, ano partially due to the extensive $\mathrm{GeH}_{4}$ gas decomposition which occurs as soon as it reaches the leading edge of the hot susceptor. Turbulence and the nonuniform stagnant boundary layer thickness result in a nonuniformity in the partial pressure of the $\mathrm{GeH}_{4}$ reactant species along the susceptor length. This, in turn, results in a continous decrease in growth rate as the distance from the leading edge of the wafer increases $(77,93)$. This effect has been reported to be reduced by tilting the susceptor at an angle relative to the gas flow direction and by increasing the total flow rate (74). In this work, the susceptor was tilted at $-15^{\circ}$ and this did not, by itself, result in a uniform growth rate. flow rates greater than $0.851 \mathrm{~min}^{-1}$ were not explored, but are a potential solution to this problem. Reduced $\mathrm{GeH}_{4}$ concentrations are also a possible solution..

The optical micrographs of figure 29 show the epilayer surf ace for three different depositions. The surface appearance varied considerably depending on the amount of time of deposition and the growth rate in the specific area. As mentioned previously, considerable nucleation and gas phase decomposition occurred at the leading edge of the susceptor. The longer the deposition time, the larger were the Ge crystals which grew on the susceptor. These cyrstals would fall, or blow, on to the growing epilayer surface. In addition, considerable gas phase nucleation caused particles to "snow" onto the wafer surface. The high growth rate at the 


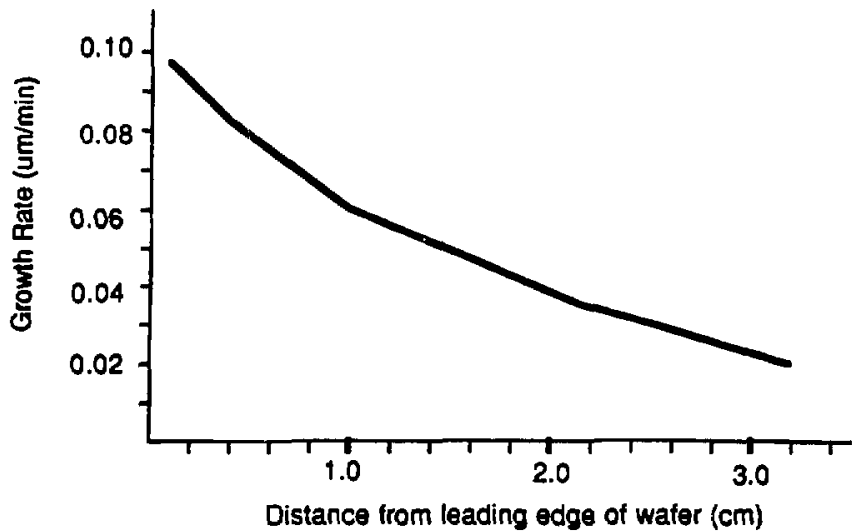

Figure 28. Growth rate of epitaxial layer as a function of distance from the front edge of the wafer for depositions in the horizontal apparatus at atmospheric pressure.

XBL $884 \quad 1758$ 


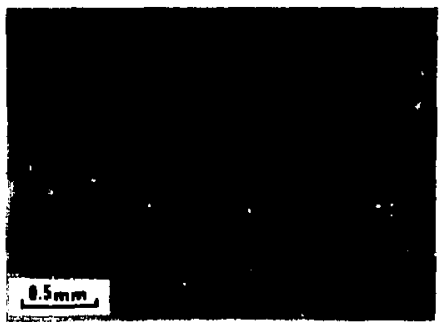

a

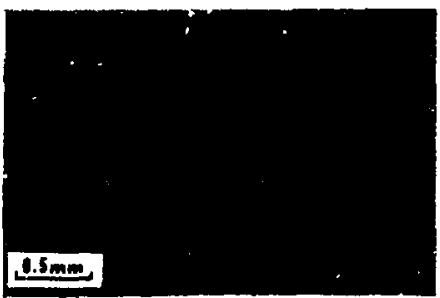

6
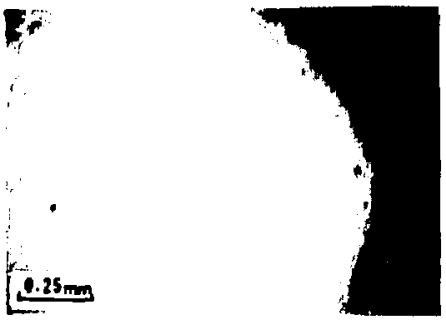

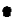

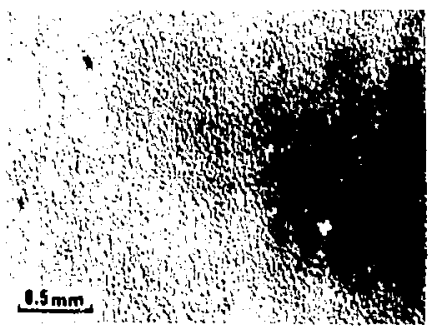

b

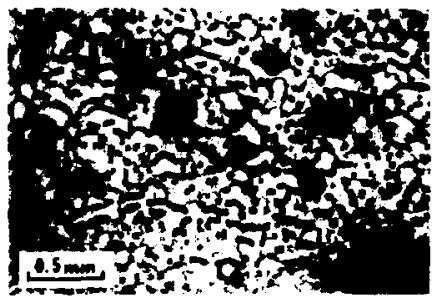

d

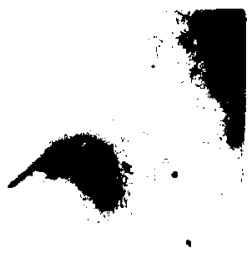

$1.25 \mathrm{~mm}$

XB8 $882-1354$

Figure 29. Optical micrographs of epilayers grown in the horizontal chamber showing the effects of deposition tinie and growth rate on epilayer quality. The layers shown in (a) and (b) are from the sane 2-hour deposition of areas where the growth rates were $-0.02 \mu n n i n^{-1}$ and $\sim 0.06 \mu m m i n^{-1}$, respectively. The layers in (c) and (d) are from an 8.5-hour deposition, again of areas where the growth rate was $\sim 0.02 \mu$ minin -1 and $\sim 0.06 \mu^{m} m^{-1}$, respectively. The layer shown in (e) and ( $f$ ) are from a 4-hour deposition where the growth rate was -0.02 ummin-1. 
leading edge resulted in a rougher surface than in areas where the growth rate was slower. Figure $29(a)$ and (b) are of the same wafer, which had a two-hour deposition time, where $29(\mathrm{a})$ is of an epilayer area farthest away from the leading edge and $29(\mathrm{~b})$ is of an area closest to the leading edge. The variation in topography as a function of distance from the leading edge of the wafer is easily observed in these photos. Similarly, Figures $29(c)$ and (d) are of the same wafer which received an eight-nour deposition. An epilayer area farthest from the leading edge is shown in 29(c) and an area closest to the leading edge is shown in 29 (d). The longer time of the deposition, as compared with 29 (a) and (b), is evident in the severe degradation of the surface. Epilayers could not be grown for more than four hours without the accumulation of crystallites and particles ruining the entire wafer surface. Figures $29(e)$ and (f) are of a highly specular area of a wafer that had a four-hour deposition time. This area was farthest from the leading edge and had a very low density of particulate-like defects, $\sim 10 \mathrm{~cm}^{-2}$. If the deposition time was kept below four hours, the extent of surface contamination from gas phase nucleation could be minimized. However, it was much more preferable that the gas phase nucleation be eliminated, instead of merely dealt with. The low pressure CVD technique, which is discussed in section 2.2.2.4, apparently solves this problem.

Figures $30(a)$ and $30(b)$ are transmission electron micrographs (TEM) of the epilayer and interface region of sample II-13. Figure 30 (a) is a cross-sectional area of the interface and the corresponding selected area diffraction pattern of the epilayer only. The epilayer is monocrystalline, but heavily dislocated, and contains submicron sized polycrystalline grains as seen by the polycrystalline ring pattern superimposed on 

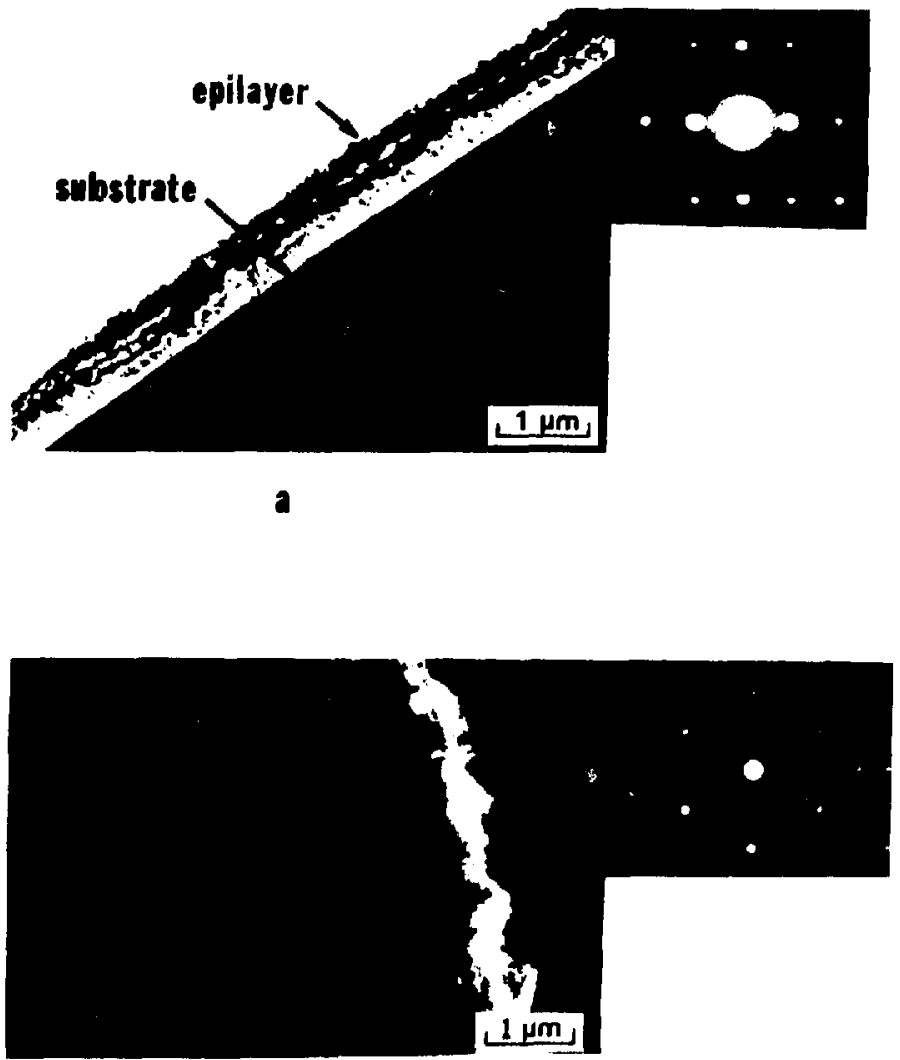

XBB $884-3386$

b

Figure 30. Transmission electron micrographs of epilayer II-13. A crosssectional ared of the epilayer/substrate interface and the respective diffraction pattern from the epilayer is shown in (a). A plan-view of the surface of the epilayer and its respective diffraction pattern is shown in (b). 
the (111) diffraction pattern. These layers were grown on (113) substrates and were oriented as such, but the TEM samples were tilted to yield the (111) diffraction pattern. The portion of epilayer shown nere is only -1 um thick, while the actual layer was $\sim 8 \mu \mathrm{m}$ thick, but the ion milling process removed much of the epilayer in this region. Figure $30(D)$ is a plan-view of the same epilayer and its corresponding diffraction pattern. The plan-view samples were made from the surface of the $\sim 8 \mathrm{um}$ thick epilayer and show that the epilayer is of a much higher crystalline quality in the surface region than close to the interface. The decrease in dislocation density with increasing epilayer thickness has been reported in the literature and is attributed to the lateral growth of the dislocations along the growing interface out to the wafer edges ano to the recombination of dislocations $(94,95)$. Measurements of the dislocation density as a function of distance from the interface were not done for this work.

\subsubsection{Sumary of Epitaxy using Horizontal Chamber}

Germanium epilayers grown in the horizontal CVD chamber at atmospheric pressure at $735^{\circ} \mathrm{C}$ were monocrystalline and very pure. The epilayers had a residual Cu concentration of $-2 \times 10^{13} \mathrm{~cm}^{-3}$ and $-10^{11} \mathrm{~cm}^{-3}$ shallow acceptors and donors. Transmission electron micrographs of cross-sectional areas of the epilayer/substrate interface revealed a high dislocation density in the epilayer within one micron of the interface, but the remaining thickness of epllayer had a very low defect density - the exact density is as yet unknown. All the epilayers grown at atmospheric pressure in the horizontal chamber suffered from 
defects due to Ge particles which nucleated in the gas phase and fell onto the growing epilayer surface. In addition, the variation in growth rate across the length of the wafer created two important problems, one being the difficulty in determining the specific area from which to fabricate a detector with an epilayer of a specific thickness, the other being the degradation in epilayer quality with increasing growth rate. Silicon epitaxial layers have been grown using a low pressure CVD (LPCVO) technique and are reported to be of equal, if not better, quality to those grown at atmospheric pressure $(35,96-98)$. The low pressure technique was first explored as a means of reducing the epitaxial growth temperature so as to minimize diffusion between adjacent layers. For Si the growth temperature can be reduced by $\sim 200^{\circ} \mathrm{C}$ while still achieving high quality layers. The lower the pressure, the easier it is for the hydrogen (in the case of $\mathrm{SiH}_{4}$ or $\mathrm{GeH}_{4}$ CVO) to desorb from the growing surface, which then allows the Si (or Ge) atoms to move more freely to energetically favorable crytallographic surface sites. Thus, the growth temperature can be decreased without compromising the mobility of the surface atcms. Low pressure CVD also results in very uniform growth rates across the wafer surface, because the reactant gas species is uniformly distrihuted by the laminar flow created by the pumping process. An additional advantage of LPCVD is the reduction, or even elimination, of gas phase nucleation, because the partial pressure of the reactant species is lower. Preliminary LPCVO studies of Ge epitaxial layers using the horizontal system discussed here, indicate that the epilayer growth rate is indeed uniform across the wafer and that gas phase nucleation is virtualiy eliminated. These layers w1ll be characterized and possibily used in BIB detectors, but to date the results are still preliminary. 
BIB detectors were fabricated from the epilayers grown on the Ge:Ga substrates described in Table II as sample numbers II-13 and II-16. These layers were as pure and were of the same crystalline perfection as those shown in the Hall data of Figure 24 and the TEM data of Figure 29, a) though those data were not specifically from those particular samples. The following section describes the fabrication and characterization of the BIB detectors which were made from these two epilayer/substrate systems.

3.0 Germanium BIB Detectors

\subsection{BIB Detector Fabrication}

A schematic of the BIB detector device with onmic contacts is shown in figure 31. The epilayer surface was implanted with boron to form an onmic contact and a copper wire was attached to the surface with silver epoxy. The opposing substrate surface was boron implanted and metallized with $-0.02 \mu \mathrm{m}$ of $\mathrm{Pd}$ and then $-0.8 \mu \mathrm{m}$ of $\mathrm{Au}$ to form the ohmic contact. Table III details the detector dimensions and implant doses for all the detectors discussed in the following sections. The substrate surface of the detector was attached to a 1 mom diameter copper post, which then fit into an absorbing metal cavity which mounted into the cryogenic photoconductor test apparatus. A photograph of a typical detector mounted in a cavity is shown in figure 32. The surface incident to the photon source was the epilayer surface for all the detectors tested. 


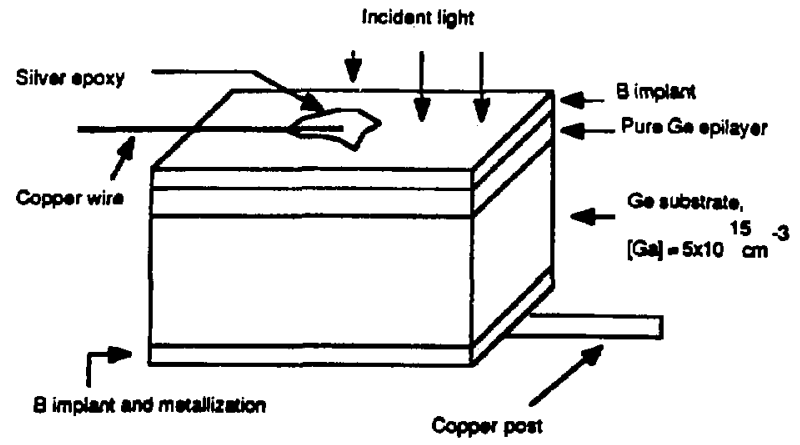

Fipure 3: Schemaic of GorGa Bus detectry dovies with chmic contects.

XBL $884 \quad 1159$ 
Table III

\begin{tabular}{|c|c|c|c|}
\hline $\begin{array}{l}\text { Detector } \\
\text { No. }\end{array}$ & $13-2$ & $13-3$ & $16-8$ \\
\hline $\begin{array}{l}\text { Heavily-doped } \\
\text { substrate, } \mathrm{cm}^{3}\end{array}$ & $\begin{array}{l}\text { Ge:Ga } \\
5 \times 10^{15}\end{array}$ & $\begin{array}{l}\text { Ge:Ga } \\
5 \times 10^{15}\end{array}$ & $\begin{array}{l}\text { Ge:Ga } \\
2 \times 1016\end{array}$ \\
\hline $\begin{array}{l}\text { Epilayer } \\
\text { thickness }\end{array}$ & $-10 \mu \mathrm{m}$ & $-10 \mu \mathrm{m}$ & $-7 \mu m$ \\
\hline $\begin{array}{l}\text { Epilayer } \\
\text { deposition nc }\end{array}$ & I I-13 & II -13 & II -16 \\
\hline $\begin{array}{l}\text { B-implant dose } \\
\text { in epilayer, } \mathrm{cm}^{-2} \text {, } \\
\text { at } 25 \mathrm{kV}: \\
\text { at } 50 \mathrm{kV}:\end{array}$ & $\begin{array}{l}1 \times 10^{14} \\
2 \times 10^{14}\end{array}$ & $\begin{array}{l}3 \times 10^{13} \\
\text { none }\end{array}$ & $\begin{array}{l}3 \times 10^{13} \\
\text { none }\end{array}$ \\
\hline $\begin{array}{c}\text { B-implant dose } \\
\text { in substrate, } \mathrm{cm}^{2} \text {, } \\
\text { at } 25 \mathrm{kV}: \\
\text { at } 50 \mathrm{kV}:\end{array}$ & $\begin{array}{l}1 \times 1014 \\
2 \times 10^{14}\end{array}$ & $\begin{array}{l}1 \times 10^{14} \\
2 \times 10^{14}\end{array}$ & $\begin{array}{l}1 \times 10^{14} \\
2 \times 10^{14}\end{array}$ \\
\hline $\begin{array}{l}\text { Anneal, after } \\
\text { implant }\end{array}$ & $\begin{array}{l}1 \text { hour } \\
250^{\circ} \mathrm{C}\end{array}$ & $\begin{array}{l}1 \text { hour } \\
250^{\circ} \mathrm{C}\end{array}$ & $\begin{array}{l}1 \text { hour } \\
250^{\circ} \mathrm{C}\end{array}$ \\
\hline $\begin{array}{l}\text { Detector } \\
\text { dimensions, mm }\end{array}$ & $\begin{array}{l}1.5 \times 1.5 \\
1.35 \text { thick }\end{array}$ & $\begin{array}{l}1.5 \times 1.5 \\
1.35 \text { thick }\end{array}$ & $\begin{array}{l}1.5 \times 1.5 \\
0.5 \text { thick }\end{array}$ \\
\hline
\end{tabular}

*Refer to Table II for details of the deposition conditions. 


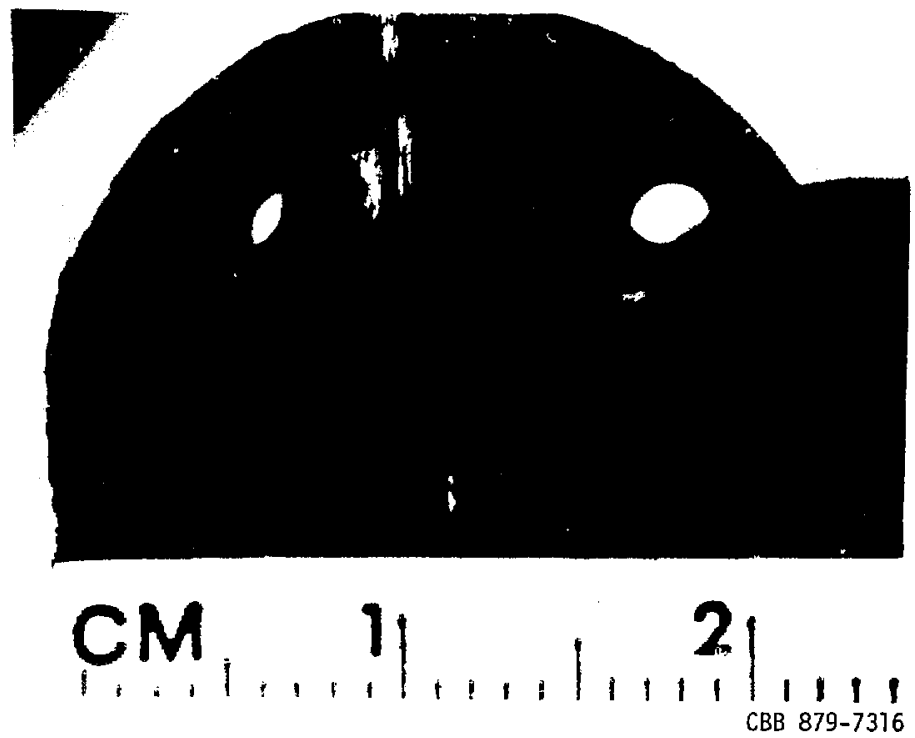

Figure 32. Photograph of a typical BIB detector mounted in a cavity. 


\subsection{Pertinent Detector Parameters}

There are many ways to characterize and test infrared detectors, but only the most useful and common ways were utilized in the characterization of the detectors studied here. For low background conditions, which is how these BIB detectors are intended to operate, the most frequently used figures of merit are dark current, responsivity and noise equivalent power (NEP). These parameters are described briefly in this section and further details can be found elsewhere (99).

The responsivity, $R$, is defined as:

$$
R=(e / h v) G_{\eta}=(e / h v)\left(v_{d} t_{\eta} / L\right) \quad\left(A W^{-1}\right)
$$

where $e$ is the charge of an electron, $h$ is Planck's constant, $v$ is the frequency of the incident photon signal, $n$ is the quantum efficiency which is the ratio of the number of absorbed photons to the number of incident photons, and $G$ is the detector gain. In a conventional detector, the gain equals the orift velocity, $v_{d}$, under a given field times the carrier lifetime, $t$, divided by the detector length, $L$. The responsivity is then expressed in $\mathrm{AW}^{-1}$, where the output signal of the detector is measured in amperes and the incident photon signal power is measured in vatts. The larger the detector responsivity, the weaker is the incident signal which produces a signal to noise ratio of one. It is generally desirable that the responsivity be maximized for optimum detector performance.

The dark current is that current which flows in ths detector under bias in the absence of an incident photon source and is measured in amt- 
peres. The dark current can be due to current leaking along surface states on the detector sides, or from thermally generated free carriers in the detector material. If the device fabrication process is optimal, the primary source of dark current will be from thermally generated free carriers. The BIB detectors studied here were fabricated in a manner which has proven very successful for conventional Ge detectors, which exhibit negligible dark current from anything other than thermally ionized carriers. This fabrication process is very reliable and thus it is believed that the primary source of dark current in the BIB device should be that from thermaliy ionized carriers from the heavily doped layer.

The NEP is a measure of the signal-to-noise ratio relative to the incident photon power:

$$
N E P=P(N / S) \quad\left(H H Z^{-1 / 2}\right)
$$

where $P$ is the incident photon signal power, $N$ is the background noise of the detector in $\mathrm{AHz}^{-1 / 2}$, and $S$ is the 6 .ector output signal in amperes. The NEP is thus a measure of the detector sensitivity. The minimum NEP, or the maximim detector sensitivity, that can be obtained is that which would occur if the only noise associated with the signal was noise due to the random arrival of incident photons and the subsequent random generation and recombination of free carriers. This is called the "background limited NEP" and was $-10^{-17}$ WHiz ${ }^{-1 / 2}$ for the confitions used in this work. 


\subsection{Detector Test Apparatus and Procedure}

The detectors were tested under low baci:ground conditions, $-10^{8}$ phcm $^{-2} \mathrm{~s}^{-1}$, to simulate the space environment that these detectors are intended to operate in. The tests were performed at liquid helium temperatures in a light-tight cryogenic dewar. The photoconductor test dewar is shown in Figure 33(a) with the signal chopper and liquid nitrogen dewar in front of the light aperature. Figure $33(b)$ shows the inside of the test dewar where the detector and appropriate filters sit on the copper cold plate which is cooled with liquid helium. Temperatures less than $4.2 \mathrm{~K}$ were achieved by pumping on the helium gas above the liquid helium. Temperatures as low as $1.4 \mathrm{~K}$ could be obtained with the existing apparatus. The liquid helium space is surrounded by a liquid nitrogen jacket, which is in turn surrounded by a vacuum space. The area where the detector sits is also under vacuum.

The modulated external photon signal was produced by $300 \mathrm{~K}$ and $77 \mathrm{~K}$ black body sources which were chopped at a frequency of $20 \mathrm{~Hz}$. Inside the dewar, both Fabry-Perot and salt filters were used to filter the external source for the desired wavelength of interest. All the detectors described in this work were tested using a combination of a $99 \mu \mathrm{m}$ FabryPerot filter and a $93 \mu \mathrm{m} \mathrm{KCl}$ reststrahlen filter (100) which, together, result in a bandwidth of $\sim 1 \mu \mathrm{m}$. These filters were chosen because they closely correspond to the peak of the spectral response of Ge:Ga. Even though the $B I 8$ detectors made from the $2 \times 10^{16} \mathrm{~cm}^{-3} \mathrm{Ge}: \mathrm{Ga}$ material had a spectral response to $180 \mu \mathrm{m}$, they also were tested with the $99 \mu \mathrm{m}$ filters, because significant response still œcurred at this wavelength and longer wavelength filters were not available at the time. 


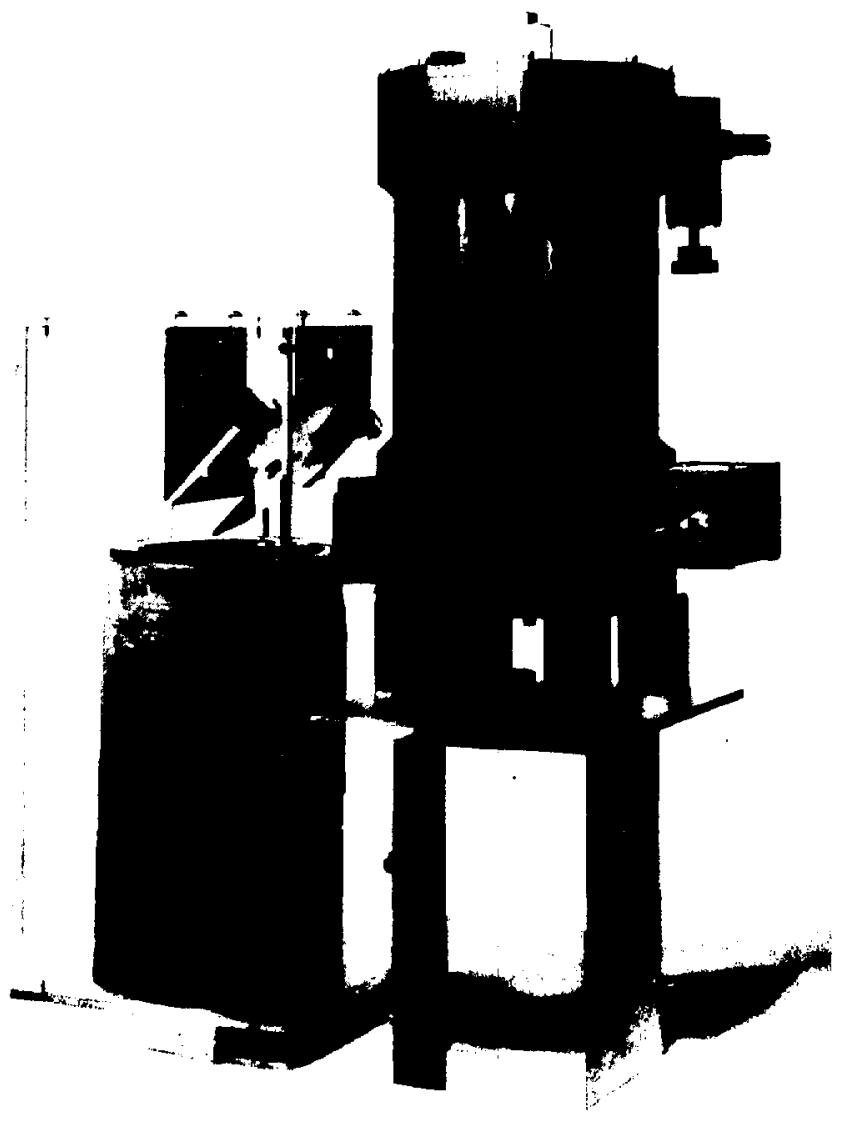

CBB $817-6972$

Figure $33(a)$. thotograph of the cryogenic test dewar. 


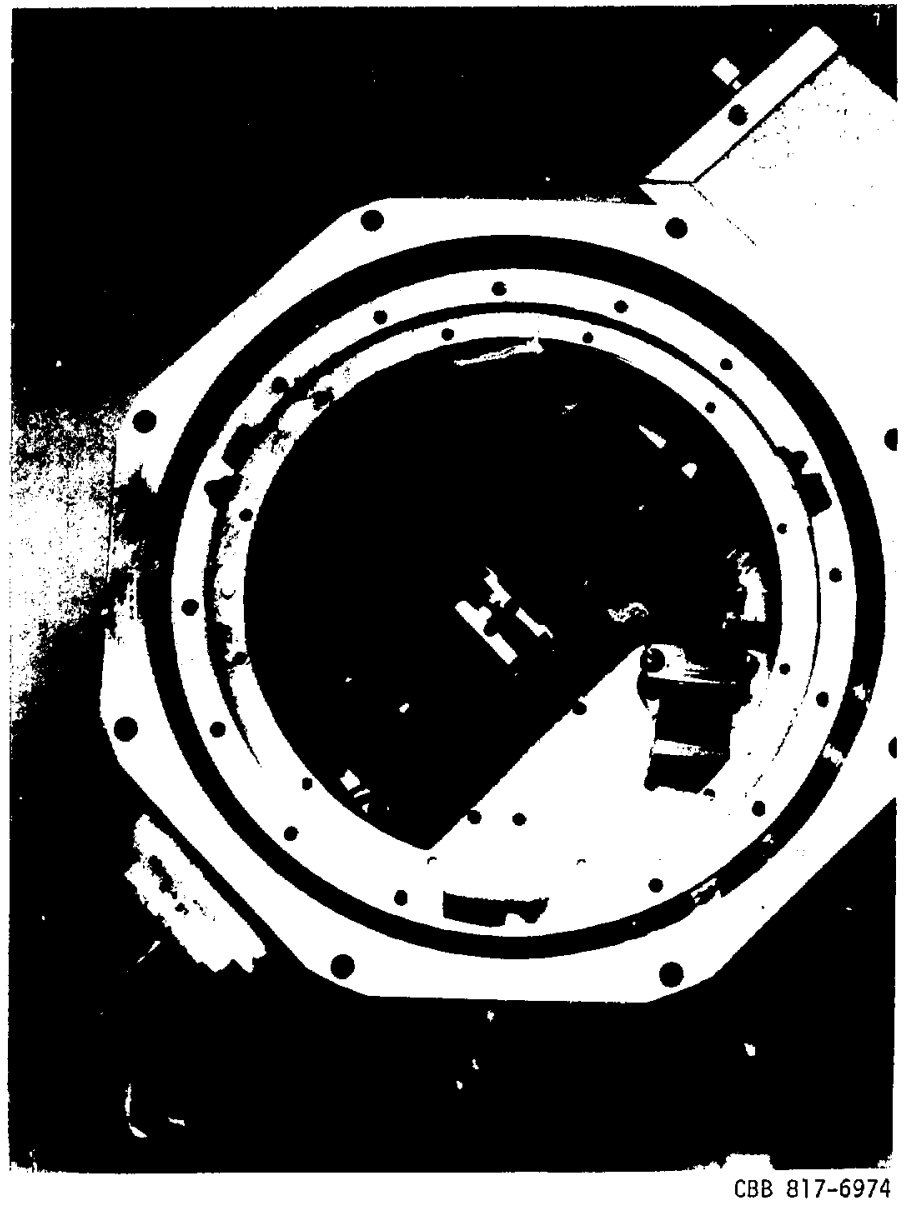

Figure 33(b). Photograph of the inside of the test dewar where the detector and filters sit on the cold plate. 
The incident photon power is a function of the incident wavelength, filter and window transmissions, diffraction losses, temperature of the black body sources, and the geometries of the aperatures and detector placement. The incident photon power for the test dewar with the $99 \mu \mathrm{m}$ and $93 \mu \mathrm{m}$ filters is the difference between the flux of the $300 \mathrm{~K} \mathrm{black}$ body and the $77 \mathrm{~K}$ black body and was equal to $2 \times 10^{-13} \mathrm{~W}$ in this case. Further details regarding the test apparatus and the calculations of the incident photon flux have been described elsewhere (11).

\subsection{Detector Results and Discussion}

Results are shown for two BIB detectors fabricated from Ge:Ga substrates which were doped with Ga at a level of $5 \times 10^{15} \mathrm{~cm}^{-3}$ and one detector fabricated from a Ge:Ga substrate doped to $2 \times 10^{16} \mathrm{~cm}^{-3}$. Each of the detectors was reproduced at least once, yielding results very similar to those shown here. The results from the detectors fabricated from the lower doped Ge:Ga material will be discussed first and the detector fabricated from the more heavily doped Ge:Ga material is discussed in Section 3.4.2.

3.4.1 Results from the $5 \times 10^{15} \mathrm{~cm}^{-3} \mathrm{Ge:Ga} \mathrm{BIB} \mathrm{Detector}$

Although a Ga doping level of $5 \times 10^{15} \mathrm{~cm}^{-3}$ was high enough that hopping conduction did occur in this material, as seen in the Hall data of Figure 21, this doping level was not high enough to result in a measureable reduction of the ionization energy of Ga in $\mathrm{Ge}$. An infrared absorption spectrum of this $5 \times 10^{15} \mathrm{~cm}^{-3}$ material is shown in Figure 
34, revealing that the onset of absorption occurs at $-80 \mathrm{~cm}^{-1}$, as it does for more lightly doped Ge:Ga materiai. BIB detectors made from this material were not expected to show an increase in spectral response over conventional Ge:Ga detectors, but the effectiveness of the epilayer as a blocking layer could still be tested, because hopping conduction does occur in this material.

\subsubsection{Dark Current}

Figure 35 shows dark current as a function of applied bias at $2.3 \mathrm{~K}$ for detector 13-2, which had a $-10 \mu \mathrm{m}$ thick epilayer and for a "detector" made from the same Ge:Ga substrate material, but without an epilayer. At low bias, $<100 \mathrm{mv}$, the detector with the epilayer exhibits a dark current two orders of magnitude less than that of the detector without the epilayer. This clearly demonstrates that the epilayer is blorking leakage current from the hopping conduction occurring in the doped IR-active region of the detector. The sharp increase in dark current which starts at $-60 \mathrm{mV}$ is due to the onset of breakdown due to impact ionization of the impurities at high fields. Table IV lists the values of electric field in this detector as a function of applied bias, and with an external bias of $60 \mathrm{mV}$, a depletion width of $77 \mu \mathrm{m}$ results and the electric field in the epilayer is $9.0 \mathrm{Vcm}^{-1}$. This field is high enough to result in the breakdown of the epilayer. The breakdown voltage of Ge is a function of majority and minority impurity concentrations, but is generally on the order of 1-10 $\mathrm{vcm}^{-1}$ (at liquid helium temperatures), and so the breakdown of the BIB detector $\propto c c u r s$ in the expected range. The operating range for these BIB detectors will have to be in the millivolt 


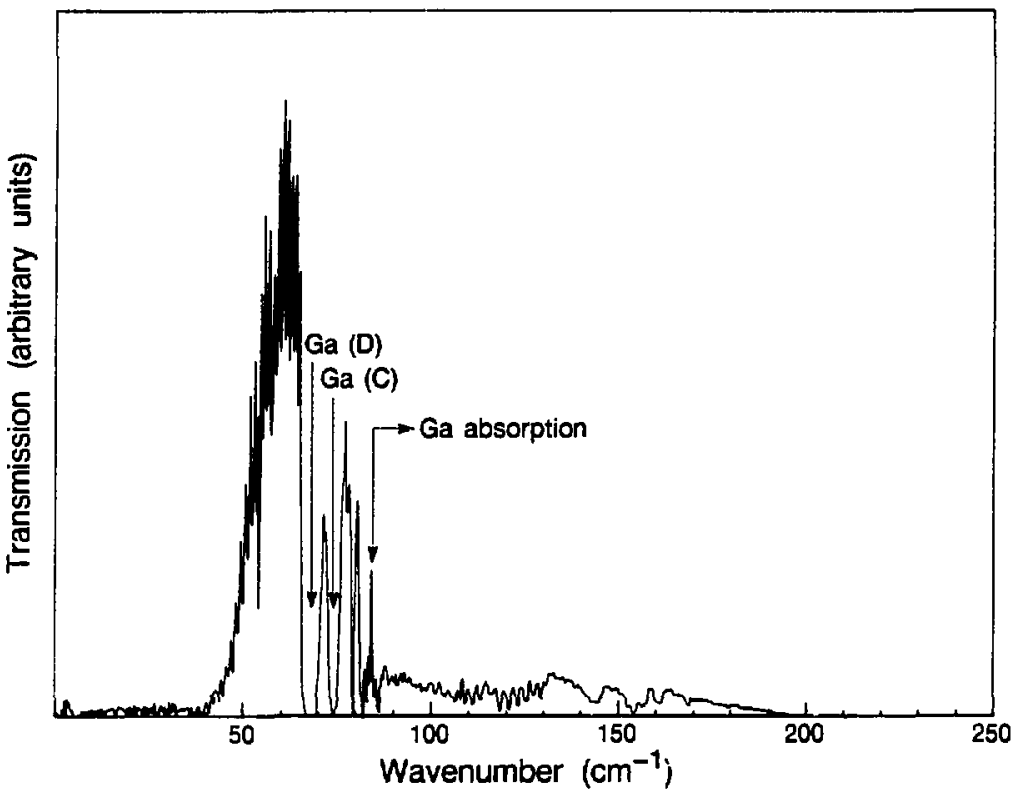

XEL 884.9604

Fiqure 34. Infrared absorption spectrum from crystal 582-11 which was used for deposition II-13 and detectors 13-2 and 13-3. 


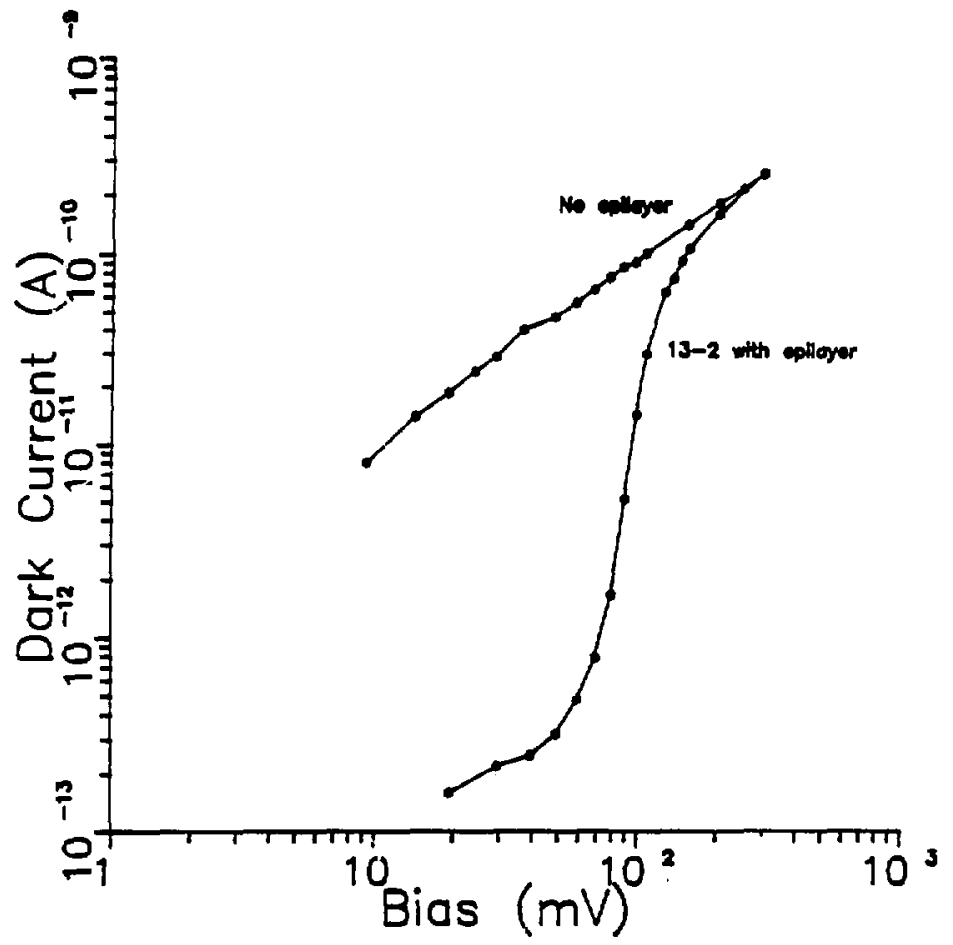

XBL 8841160

Figure 35. Dark current as a function $c^{-}$detector bias for oetector 13-2 with an epilayer and for the same "detector" without an epilayer at $2.3 \mathrm{~K}$ under reverse bias. 
Table IV

\begin{tabular}{|c|c|c|c|c|c|c|}
\hline $\begin{array}{c}\text { Detector } \\
\text { Ho. }\end{array}$ & $\begin{array}{l}\text { Epilayer } \\
\text { thiekness, }\end{array}$ & $\begin{array}{c}\text { Residual donor } \\
\text { concentryation, } \\
\mathrm{cm}\end{array}$ & $\begin{array}{l}v_{a}^{\prime} \\
m v \\
\end{array}$ & $\begin{array}{c}V_{a}-V_{b i}^{2}, \\
m v\end{array}$ & $\begin{array}{c}\text { Depletijon } \\
\text { width? } \\
\text { um }\end{array}$ & $\begin{array}{l}\text { Field, } \\
\mathrm{V} \mathrm{cm}^{-1} \\
\end{array}$ \\
\hline $\begin{array}{l}13-2 \\
\text { and } \\
13-3\end{array}$ & -10 um & -1010 & $\begin{array}{l}-20 \\
-30 \\
-40 \\
-50 \\
-60\end{array}$ & $\begin{array}{l}-3.6 \\
-13.6 \\
-23.6 \\
-33.6 \\
-43.6\end{array}$ & $\begin{array}{l}17 \\
39 \\
54 \\
66 \\
77\end{array}$ & $\begin{array}{l}1.9 \\
4.6 \\
6.4 \\
7.8 \\
9.0\end{array}$ \\
\hline $16-8$ & -7 um & $-5 \times 10^{11}$ & $\begin{array}{l}-20 \\
-30 \\
-40 \\
-50 \\
-60\end{array}$ & $\begin{array}{l}-1.6 \\
-11.6 \\
-21.6 \\
-31.0 \\
-41.6\end{array}$ & $\begin{array}{l}0.3 \\
2.3 \\
4.0 \\
5.4 \\
6.7\end{array}$ & $\begin{array}{l}2.2 \\
14 \\
24 \\
32 \\
40\end{array}$ \\
\hline
\end{tabular}

1. $V_{a}$ is the externally applied bias.

2. $V_{b i}$ is the built-in voltage (see Appendix I).

3. Depletion width is calculated from equation (2) in text, with $c=15.4$ for Ge and $c_{0}-8.85 \times 10^{-12}$ faradm 1 .

4. The field listed is that in the epilayer, which is also the maximum in the depletion layer. The field in the depletion layer is a function of depletion depth, and is constant as a function of bias. It is equal to $1.2 \times 10^{3}(x)$ for detectors $13-2$ and $13-3$ and is equal to $6.0 \times 10^{4}(x)$ for detector 16-8, where $x$ is the distance into the depletion layer in cm. See Appendix II for the calculations of electric field. 
range, because of the chin region over which the electric field occurs. Watson and Huffman also observed breakdown in their Ge:Ga BIB detectors at $-50 \mathrm{mV}(31)$.

Figure 36 shows dark current for detector $13-2$ at $2.3 \mathrm{~K}$ in both the forward and reverse bias configurations. The epllayer is negative in the reverse biased configuration and this is the proper bias for a p-type BIB device in order for the pure layer to functi ... as a blocking layer. In the forward bias configuration, the epilayer does not block leakage current from hopping conduction in the impurity band, as holes are easily injected from the positive contact, because of the steep potential gradient in the epilayer. Figure 5 schematically details the space charge, electric field and potential band diagram for a forward biased BiB and shows the steep potential gradient in the epilayer. (The reverse bias case is shown in Figure 4). Returning to Figure 36, it is seen that the dark current of the detector is much higher in the forward bias direction than in the reverse bias direction, as would be expected if the epilayer were functioning properly.

The dark current of the BIB detector decreases as a function of temperature, as shown in Figure 37, which is of detector 13-2 at $2.5 \mathrm{~K}$ and $2.3 \mathrm{~K}$. The dark current decreases with temperature, because fewer carriers are thermally ionized at lower temperature. Temperatures lower than $2.3 \mathrm{~K}$ were not explored, but the dark current could be reduced substantially with further reductions in temperature. For example, a dark current of $10^{-16} \mathrm{~A}$ is predicted at $1.8 \mathrm{~K}$ for these detactors (see Appendix III). The lowest values seen here, $-10^{-13} \mathrm{~A}$, are approximately 10-100 times higher than standard conventional Ge:Ga detectors al though the conventional detector technology is improving rapidly and 


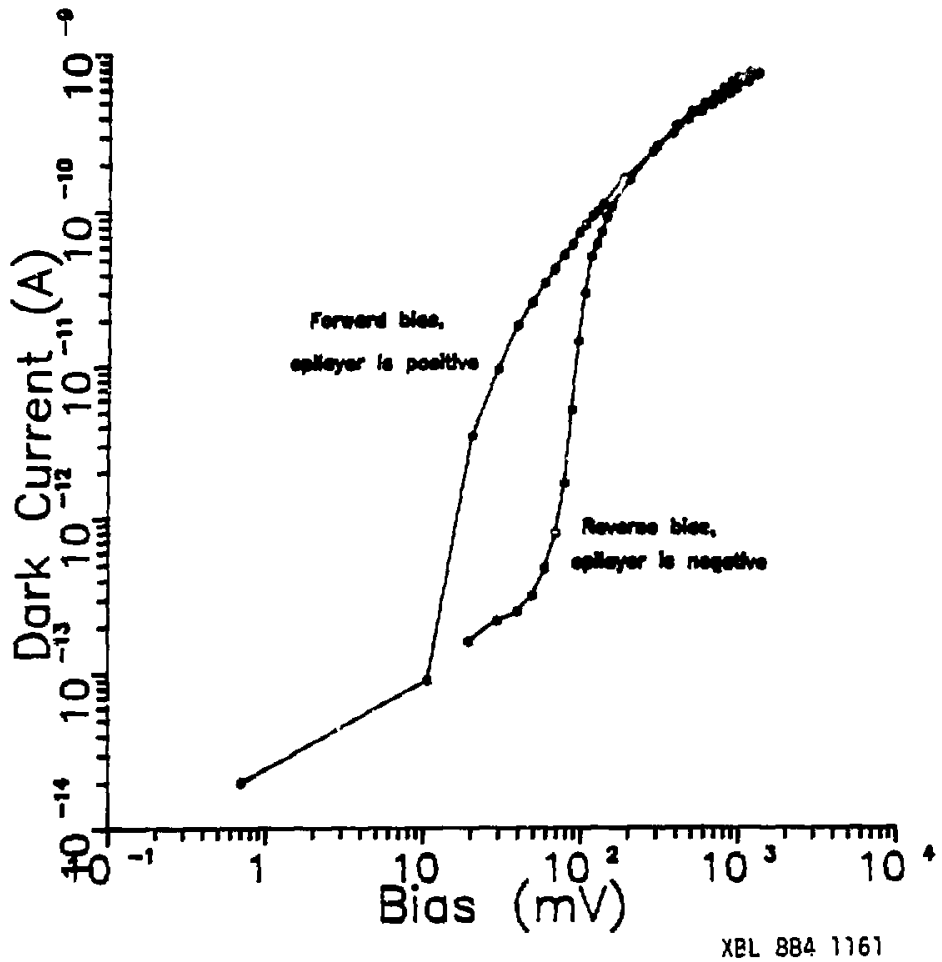

Figure 36. Dark current as a function of bias for detector $13-2$ at $2.3 \mathrm{X}$ for both the forward and reverse bias configurations. 


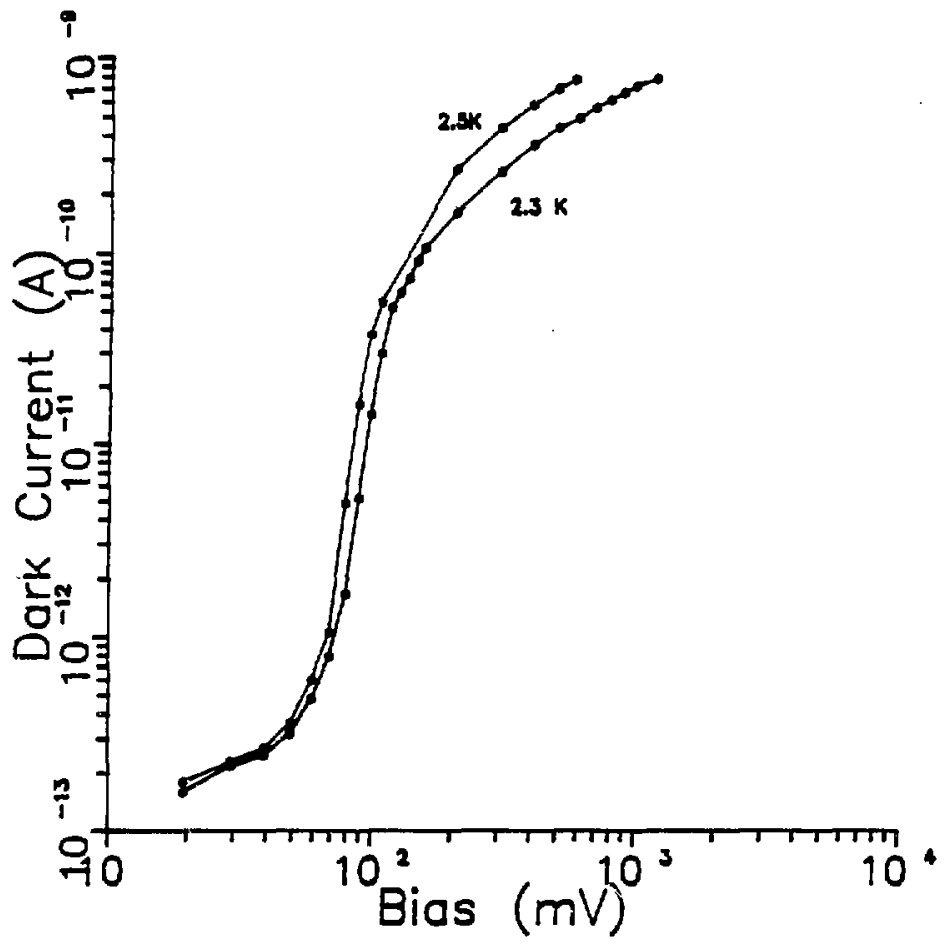

XBL $884 \quad 1162$

Figure 37. Dark current as a function of bias for detector 13-2 at $2.3 \mathrm{~K}$ and $2.5 \mathrm{~K}$ under reverse bias. 


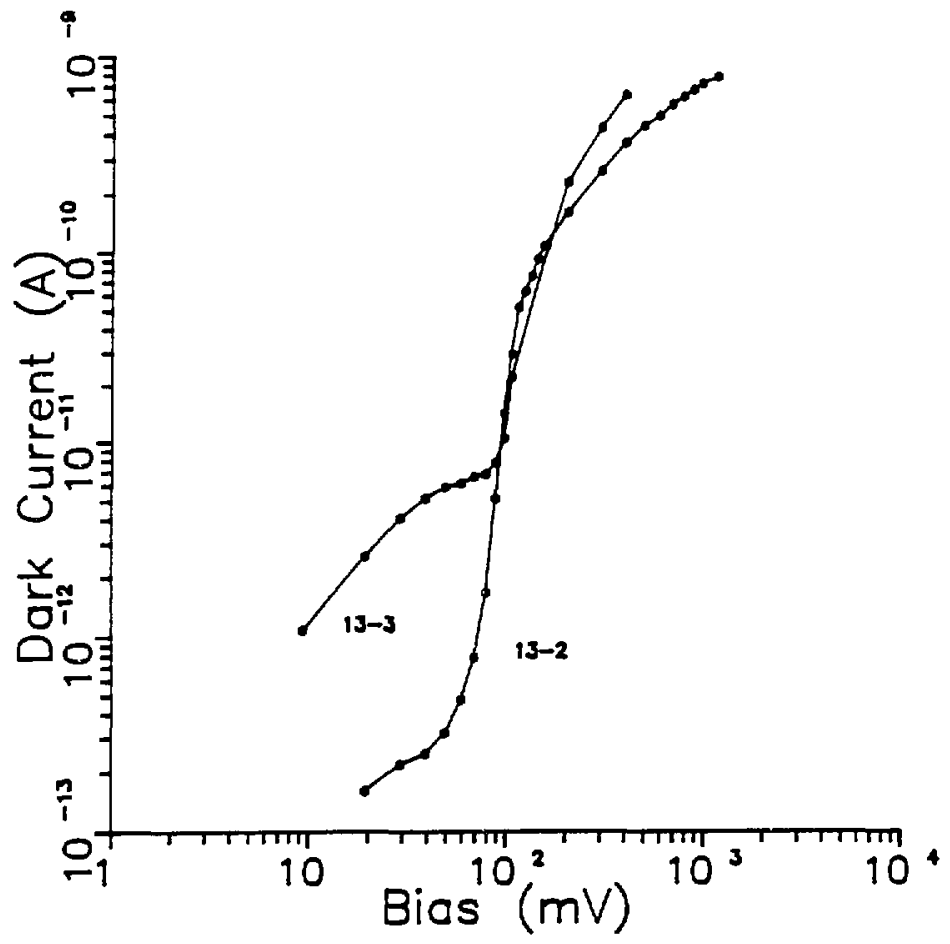

XCL 8341163

Figure 38. Dark current as a function of bias for detectors 13-2 ana 13-3 at $2.3 \mathrm{~K}$ under reverse bias. 


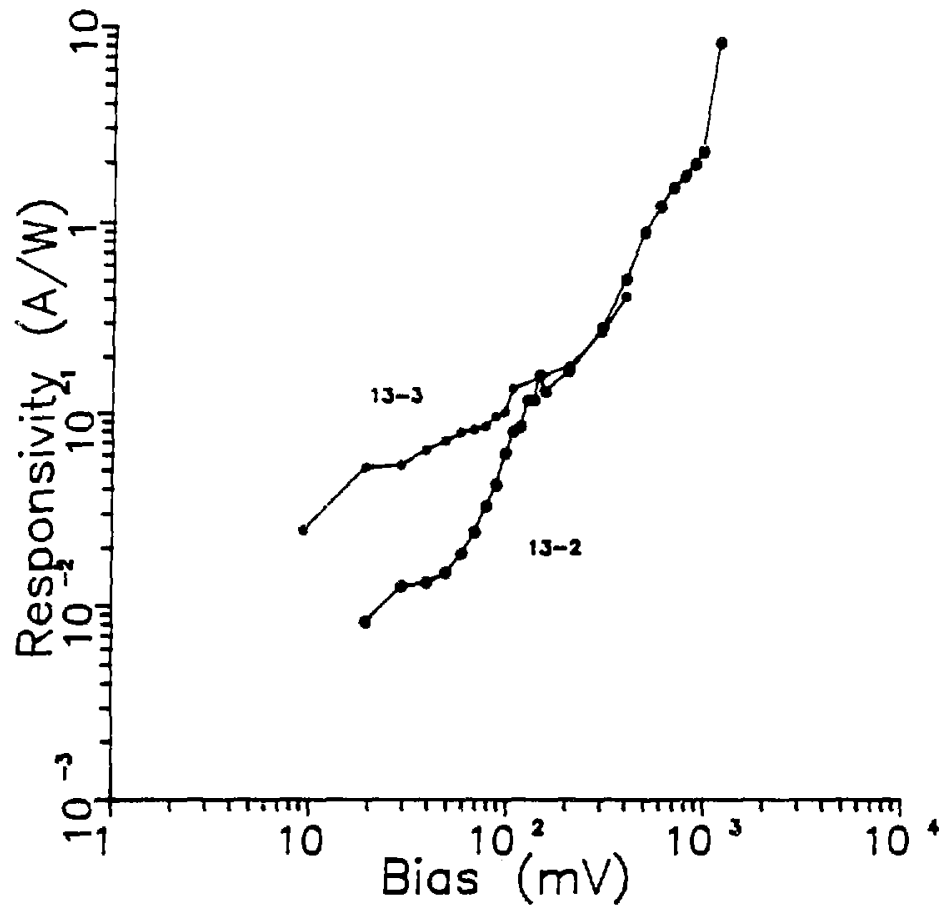

XEL $884 \quad 1164$

Figure 39. Responsivity as a function of bias for getectors $13-2$ ano $13-3$ at $2.3 \mathrm{~K}$ under reverse bias. 
$3 \times 10^{13} \mathrm{~cm}^{-2}$ at $20 \mathrm{kV}$ has a $45^{\circ} / 0$ transmittance (not incluaing the surface reflectance due to the refractive index of $\mathrm{Ge}$ ), while a heavier dose of $3 \times 10^{14} \mathrm{~cm}^{-2}$ at $20 \mathrm{kV}$ has a $35 \%$ transmittance (102). These doses are similar to those given detectors 13-3 and 13-2, respectively, and hence the transmittance of the implant on detector 13-3 would be expected to be greater than that of detector 13-2. The responsivities exhibited by these detectors are on the order of 10-100 times lower than those of conventional Ge:Ga detectors (11).

The responsivity of detector $13-3$ at $2.2 \mathrm{~K}$ as a function of bias for both the forward and reverse configurations is shown in figure 40 . Responsivity is a direct function of gain, $G$, and quantum efficiency, $n$, and so $\mathrm{G}_{n}$ is also shown as a function of bias for the same detector in Figure 41. Although the reverse bias configuration is the proper configuration for p-type BIB detectors, the responsivity is less when the detector is reverse biased than when it is forward biased, as Figure 40 shows. Correspondingly, the $G_{n}$ factor is also less in the reverse bias configuration. This is due to the large difference in current and electric field in the two bias configurations. When reverse biased, the electric field occurs in the epilayer and in the depletion layer, as shown in Figure 4(c). For this detector, the epilayer plus depletion layer is only $63 \mu \mathrm{m}$ at $-40 \mathrm{mV}$, as seen from Table IV, and so only those carriers generated in this $63 \mu \mathrm{m}$ region can be collected and detected as the output signal of the detector. The absorption length for Ge:Ga at $100 \mathrm{~cm}^{-1}$ is $-200 \mu \mathrm{m}$ for $[\mathrm{Ga}]=5 \times 10^{15} \mathrm{~cm}^{-3}(103)$, and so a $63 \mathrm{\mu m}$ absorbing region does not absorb all the incident photons. In fact, the depletion region may be even thinner than calculated, if the impurity band is not fully formed, as it may not be in this lower doped material. The undepleted IR-active 


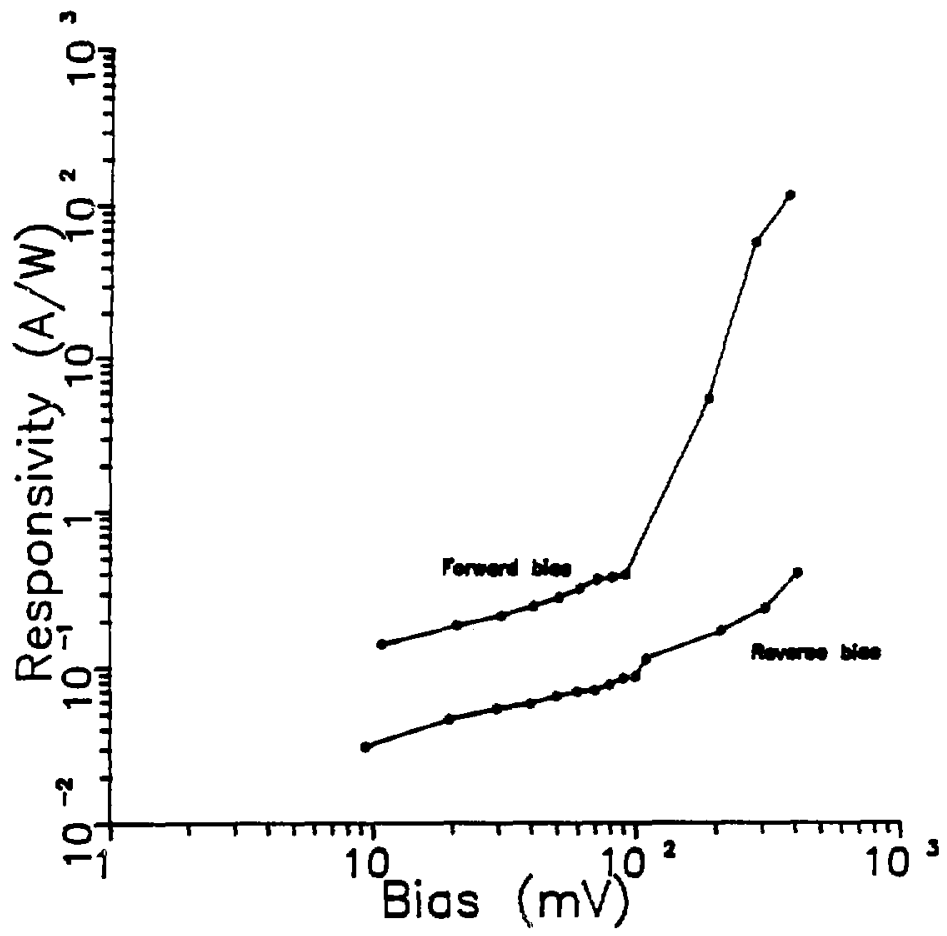

XBL 8841165

Figure 40. Responsivity as a function of bias for detector $13-3$ at $2.2 \mathrm{~K}$ for both forward and reverse biases. 


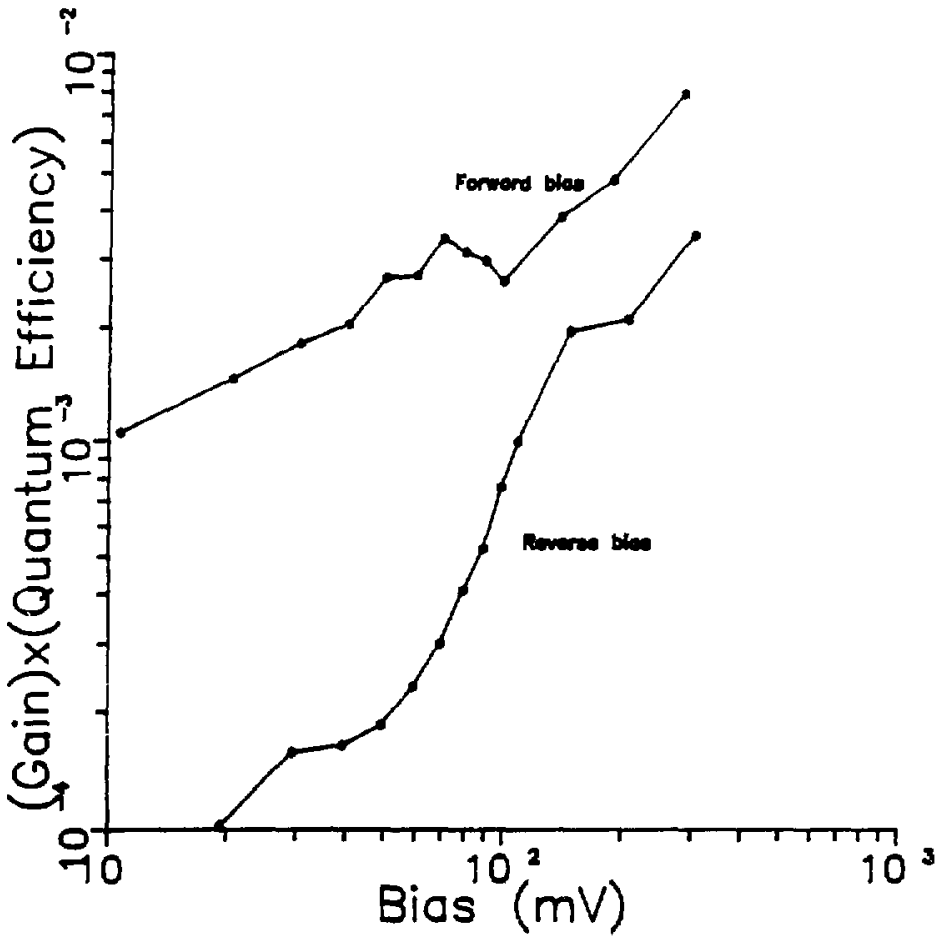

XBL $884 \quad 11 E 6$

Figure 41. Gain-quantum efficiency product as a function of bias for aetector 13-3 at $2.2 \mathrm{~K}$ for both forward and reverse biases. 
region of the detector will absorb photons, but photogenerated carriers will scarcely be detectable, because there is a very little electric field in that low resistive region. In comparison, when the detector is forward biased, there is a low electric field in the entire detector, as shown in figure $5(c)$. The larger voltage drop in the forward bias case, compared to the reverse bias case, is due to the much larger current. The larger voltage drop and higher electric field results in both an increased quantum efficiency and also an increased gain, both of which contribute to the increased responsivity of the forward biased detector. Operation in the forward bias mode is, of course, not acceptable because of the excessive current. It has been used for analytical purposes only.

The depletion region which forms in the lower doped material under reverse bias is, at best, a fraction of the total detector length. Because significant $A^{-}$charge transfer does not occur, the gain will always be less than one. The gain can be increased to unity by making the devices thinner - as thin as the depletion region plus epilayer. If the $G$ f factor were measured in such a thin device, ano assuming it behaved ideally with no recombination of carriers occuring in the heavily doped region or in the epilayer, then the quantum efficiency could be determined quantitatively. Conversely, if the quantum efficiency were predetermined, as it would be when using a very thin device in an integrating cavity (101), then the gain could be determined quantitatively. A measurement of unity gain would confirm the existence of the depletion layer. In the measurements made here, the individual values of $G$ and $n$ could not be separated, because the devices were much thicker than the depletion width and the cavities used were not the integrating type. The fixed gain theory cannot be tested quantitatively unless the noise 
levels are below background limited conditions, and the dark currents were well above background limited noise for these detectors. Even though the responsivity was higher in the forward bias case, which might appear to be desirable, the dark current is also higher and so this is not an acceptable bias configuration. It is imperative that the dark currents be as low as possible for space applications, and actually must be on the order of hundreds of electrons per second, $-10^{-17} \mathrm{Amm}^{-2}$.

\subsubsection{Noise Equivalent Power}

Noise equivalent power (NEP) as a function of bias is shown in Figure 42 for detectors $13-2$ and $13-3$ at $2.3 \mathrm{~K}$ under reverse bias. The smaller values of NEP at Iow bias for detector 13-3 compared with 13-2 are directly related to the larger responsivities for detector 13-3, as discussed in the previous section. The larger the output signal, the smaller the MEP, and detector 13-3 had higher output signals than 13-2 due to the more transparent contact. The minimum NEP values occur at biases less than $-80 \mathrm{mV}$, which is prior to breakdown. As soon as the device breaks down, the noise increases, which increases the NEP. The slow decrease in NEP after breakdown occurs is due to the increase in output signal. However, the dark current increases dramatically after breakdown, so any decrease in MEP and increase in responsivity are far outweighed by the dark current considerations.

\section{4 .2 Results from the $2 \times 10^{16} \mathrm{~cm}^{-3}$ Ge:Ga BIB Detector}

As detailed in Table [II, detector 16-8 consisted of an epilayer 


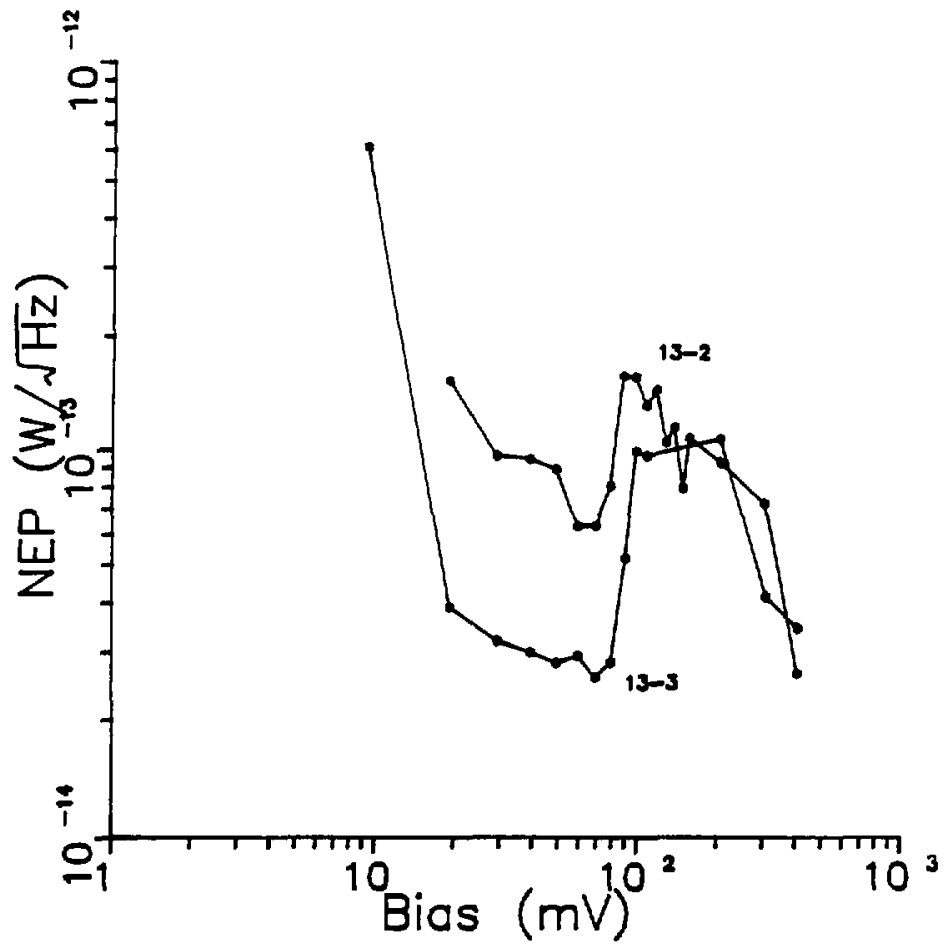

XBL $884 \quad 1167$

Figure 42. NEP as a function of bias for detectors $13-2$ and $13-3$ at $2.3 \mathrm{~K}$ under reverse bias. 
grown on a Ge:Ga substrate containing a Ga concentration of $2 \times 10^{16} \mathrm{~cm}^{-3}$. This doping level is four times higher than for the two detectors discussed in the previous sections. The $2 \times 10^{16} \mathrm{~cm}^{-3}$ Ge:Ga material exhibited extensive hopping conjuction, as shown previously in the Hall effect lata of Figure 21 . The infrared absorption spectrum from this material, shown in Figure 43 , reveals that the onset of absorption occurs at $-55 \mathrm{~cm}^{-1}$ which corresponds to an average ionization energy $0.007 \mathrm{eV}$. This is a reduction of $0.004 \mathrm{eV}$ from discrete $\mathrm{Ga}$ levels in Ge. The onset of absorption at $55 \mathrm{~cm}^{-1}$ corresponds to a maximum detectable wavelength of $180 \mu \mathrm{m}$, which as an increase of $-60 \mu \mathrm{m}$ over the $5 \times 10^{15} \mathrm{~cm}^{-3}$ material used for detectors $13-2$ and 13-3. Significant absorption still occurs at $100 \mathrm{~cm}^{-1}$ and so, again, the $100 \mathrm{um}$ filters were used when testing detectors made from the more heavily doped material.

\subsubsection{Dark Current}

Figure 44 shows dark current as function of bias for detector 16-8 at $1.4 \mathrm{~K}$ for both the forward and reverse bias configurations. The dark current is essentially linear and is essentially the same for both biases, unlike that seen for detector 13-2 under the same conditions. The fact that the dark current is the same for both bias configurations indicates that either the epilayer is not blocking the hopping conduction and/or the dark current is due to thermally ionized carriers. The blocking layer is most probably functioning properly in blocking hopping conduction, because: 1) the layers were the same as those grown for the previous detectors, and 2) four detectors made from two different epitaxy 


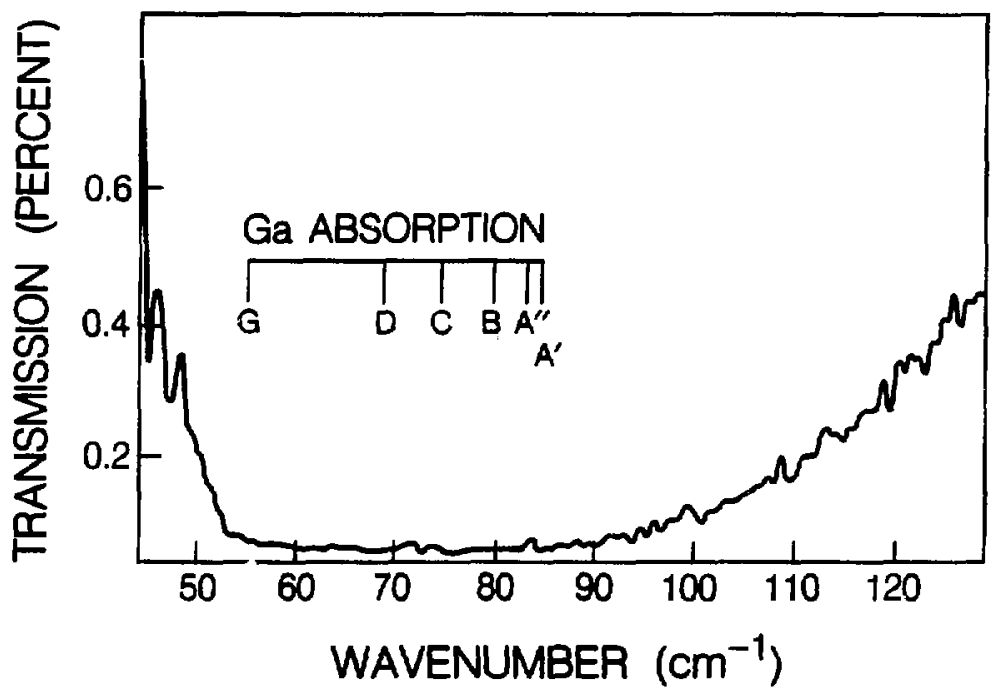

XBL 885-8888

Figure 43. Infiared absorption spectrum from crystal 746-14 which was used for depositions II-14 and II-16 and for detector 16-8. The continuous absorption from $55 \mathrm{~cm}^{-1}$ through $90 \mathrm{~cm}^{-1}$ indicates the overlap of the individual Ga wave functions. 


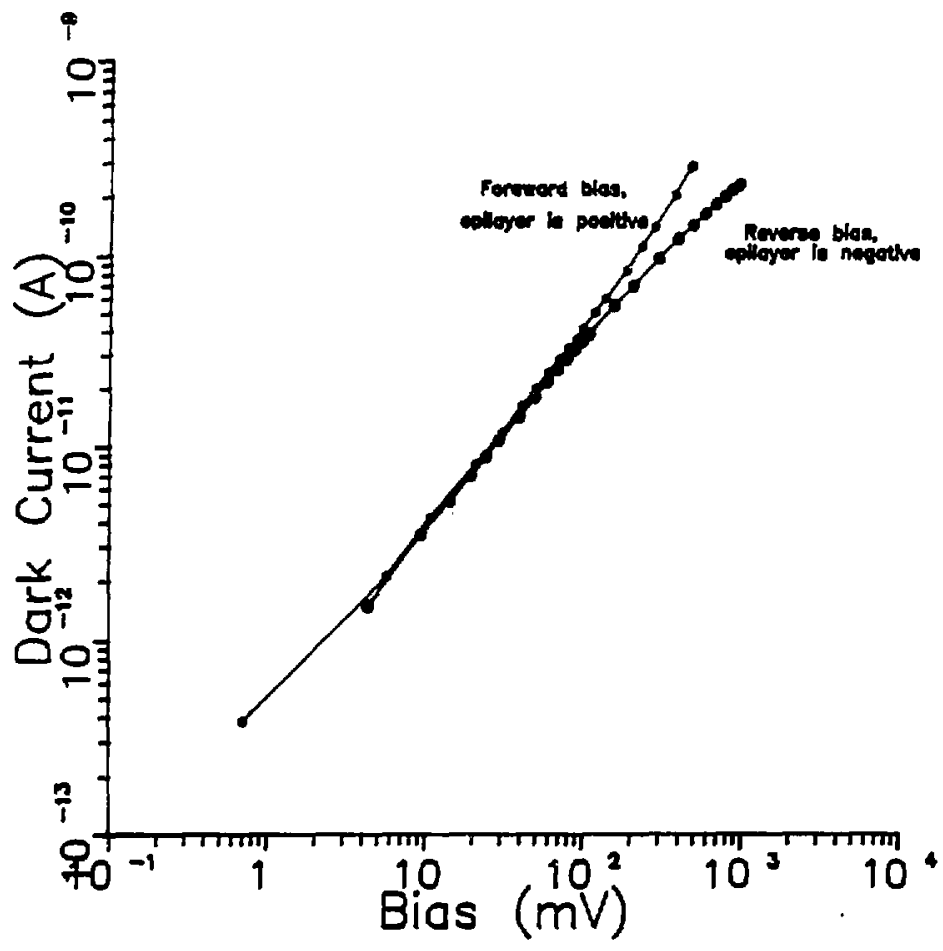

XRL 8341169

Figure 44. Dark current as a function of bias for detector $16-8$ at $1.4 \mathrm{x}$ for both forward and reverse bias. 
depositions on the $2 \times 10^{16} \mathrm{~cm}^{-3}$ substrates all yielded the same ark current characteristics. These factors indicate that something ntner than a non-functional blocking layer is most likely occurring. It is possible that breakdown occurs at very low bias, because the electric field in the epilayer is much higher for the 16-8 detector than for the 13-3 detector at equivalent bias, as detailed in Table IV. The linear bias dependence of the dark current is most frobably due to a combination of breakdown and conduction from thermally ionized carriers from the heavily doped IR-active layer. Even though the number of thermaliy ionized carriers does decrease with temperature for this heavily doped material, as seen in the dark current data taken at $3.0 \mathrm{~K}$ and $1.4 \mathrm{~K}$ of Figure 45, there is apparently still a large number of carriers in the valence band at temperatures as low as $1.4 \mathrm{~K}$.

As is detailed in Appendix I, the number of free holes in the heavily doped material is calculated from the measured dark current value of $5 \times 10^{-12} \mathrm{~A}(20 \mathrm{mV}, 1.4 \mathrm{~K})$ to be $6.3 \times 10^{4} \mathrm{~cm}^{-3}$ and the fermi level is calculated to be $0.003 \mathrm{eV}$ above the valence band. This value is very close to the theoretical Fermi level for such heavily doped materiat. Using the experimentaliy determined Fermi energy, a tempsrature of $1 \mathrm{~K}$ is predicted to be required to reduce the dark current from thermally ionized carriers to $10^{-16}$ A (Appendix III). These detectors were not tested at temperatures less than $1.4 \mathrm{~K}$, as this was the minimem that could be achieved with the existing apparatus. Although calulations of free hole densities based on Fermi energy considerations are useful, one should really know the density of states between the valerica band edge and the impurity band in order to accurately predict how the free hole density decreases with temperature. Unfortunately, the density of states 


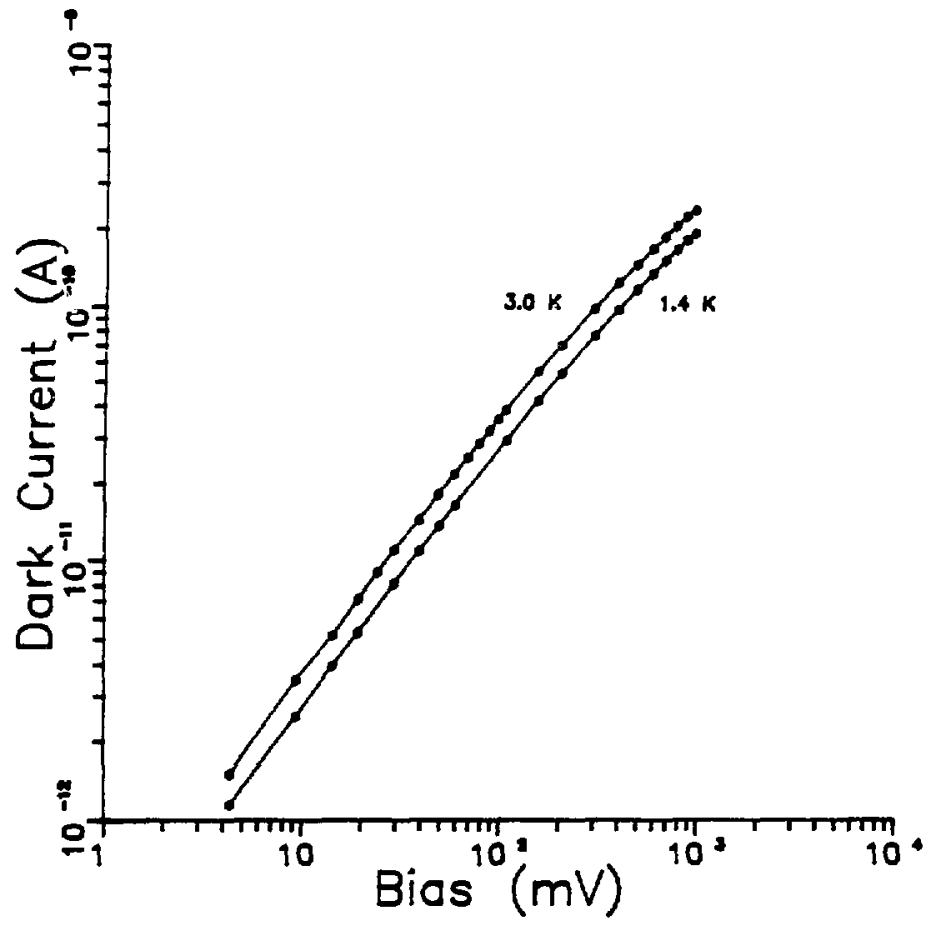

XBL $C 841170$

Figure 45. Dark current as a function of bias for detector 16-8 under reverse bias at $3.0 \mathrm{~K}$ and $1.4 \mathrm{~K}$. 
function can only be approximated, based on existing models, and so it is not known quantitatively for this material. The density of states model for a doped semiconductor with very low compensation proposed by Shkolvskii and Efros (20) predicts, 1) that the spread in energy due to impurity banding is extremely small for the $2 \times 10^{16} \mathrm{~cm}^{-3} \mathrm{Ge}: \mathrm{Ga}$ material and, 2) that the density of states does fall to a negligible value within $0.001 \mathrm{eV}$ of the impurity energy leve1 (see Appendix IV). This same model predicts a Fermi energy of $0.004 \mathrm{eV}$ for the $2 \times 10^{16} \mathrm{~cm}^{-3}$ material, which is quite close to the 0.003 eV value determined from the experimental dark current measurements. Thus, based on preliminary dark current measurements and from considerations of the fermi level and predicted density of states, it appears that an operating temperature of $-1 K$ is required for these heavily-doped BIB detectors. This will have to be verified by measuring the dark current in detectors at such low temperatures.

\subsubsection{Responsivity}

Detector 16-8 exhibited no detectable output signal and hence no responsivity or NEP could be measured. This is thought to be due to a combination of two factors: First, because of the higher compensation in the IR-active layer of detector 16-8 compared with detectors 13-2 and 13-3, it is calculated from equation (2) that detector 16-8 had a thinner depletion region than the other detectors. As shown in Appendix II, the thin depletion widths cause a very high electric field in the epilayer even at low bias. These epilayers probably break down at much lower biases than those of detectors 13-2 and 13-3. The leakage current from 
breakdown and from the thermally ionized carriers probably swamps any signal from photon absorption in the very thin depletion layer. In order to detect any signal, $G$ must be increased by increasing the depletion width and the noise level must be reduced. The depletion width can be increased by decreasing the concentration of compensating donors in the heavily doped IR-active layer and by decreasing the epilayer thickness. The noise level can be decreased by reducing the operating temperature which reduces the number of thermally generated carriers, and by reducing the electric field in the epilayer to shift breakdown to higher biases. The lat ar can be achieved by increasing the depletion width.

\subsection{Conclusions}

Germanium blocked impurity band (BIB) far infrared photoconduc:ive detectors have been successfully fabricated and characterized. Each BIB detector studied in this work consisted of a pure Ge epitaxial layer, grown by atmospheric pressure CVO using $\mathrm{GeH}_{4}$, on a Ge:Ga substrate. Detectors were made from Ge:Ga substrates with a Ga concentration of $5 \times 10^{15} \mathrm{~cm}^{-3}$ and a compensation level of $10^{10} \mathrm{~cm}^{-3}$, and were also made from Ge:Ga substrates doped to $2 \times 10^{16} \mathrm{~cm}^{-3}$ with a compensation level of $5 \times 10^{11} \mathrm{~cm}^{-3}$. The epitaxial layers were very pure, containing a residual $\mathrm{Cu}$ concentration of $-2 \times 10^{13} \mathrm{~cm}^{-3}$ and were monocrystalline. Although the layers were monocrystaliine, they contained a high defect density within one micron of the substrate/epilayer interface, but had a much lower density of defects in the remaining $-10 \mu \mathrm{m}$ of epilayer. The defect densities have not yet been determined quantitatively. In addition, the epilayers contained a visible surface defect density of 
$-10 \mathrm{~cm}^{-2}$ which resulted from Ge crystallites nucleating in the gas phase during the epitaxy deposition process. Future epitaxy work will include: 1) the elimination of defects due to gas phase nucleation by employing low pressure CVO methods, 2) the correlation of the effects of the high dislocation density at the interface with detector device performance, and 3 ) the reduction in the interface dislocation density by introducing an in-situ $\mathrm{HCl}$ etch during the CVD process immediateiy prior to Ge deposition, which will ensure a clean substrate surface.

As predicted, the pure epitaxial layers appeared to block the hopping conduction which occurred in the heavily doped IR-active layer of the BIB device when the detector was biased. The BIB detectors made from the $5 \times 10^{15} \mathrm{~cm}^{-3} \mathrm{Ge}: \mathrm{Ga}$ substrates exhibited assymmetrical device characteristics, such as dark current, responsivity and NEP, when forward and reverse biased. This indicated that the epilayer was highly resistive and most probably functioning effectively as a blocking layer. The dark currert, responsivity and NEP of the $5 \times 10^{15} \mathrm{~cm}^{-3}$ Ge:Ga BIB detectors were respectable compared with conventional Ge:Ga detectors, but require further optimization. The detectors made from the $2 \times 10^{16} \mathrm{~cm}^{-3} \mathrm{Ge}: \mathrm{Ga}$ material suffered from high dark currents, even at temperatures as low as $1.4 \mathrm{~K}$, due to the large number of thermally ionized carriers in this heavily doped material. The effects of epilayer thickness, doping concentration and compensation of the heavily doped layer, detector dimensions and operating temperature on device performance need to be evaluated carefully. The effects of these parameters on detector performance have not yet been systematicaliy investigated.

In brief, this work demonstrates that Ge:Ga 818 detectors can be fabricated and that they do perform as infrared detectors, but $f$ urther 
work is required to optimize the device characteristics. Their predicted characteristics of extended spectral response, fixed unity gain and noise reduction, compared with conventional detectors, have not yet been fully demonstrated, but there is plenty of room for further experimentation. The preliminary results to date are very encouraging, especially in conjunction with the results from Watson and Huffman (31), and further research is warranted. 
1. D.S. Evans, ed., Herschel At the Cape: Diaries and Corresponoence of Sir John Herschel, University of Texas Press, Austin, Texas, (1969).

2. G.H. Rieke, M.W. Werner, R.I. Thampson, E.F. Becklin, W.F. Hoffman, J.R. Houck, F.J. Low, W.A. Stein and F.C. Witteborn, "Infrared Astronomy After IRAS", Science, 231, 807 (1986).

3. F.C. Gillett and M.W. Werner, NASA Technical Memo. 86663 (1984).

4. P.L. Richards and L.T. Greenberg, "Infrared Detectors for Low Background Astronomy: Incoherent and Coherent Devices from One Micrometer to One Millimeter", in Infrared and Millimeter Waves, K.J. Button, ed., Academic Press, New York, 6 , 150 (1981).

5. E.E. Haller, M.R. Hueschen and P.L. Richards, App1. Phys. Lett., 34, 495 (1979).

5. R.H. Bube, Photoconductivity of Solids, John Wiley and Sons, Inc., New York, $(1960)$.

7. R.M. Brovdy and V.J. Mazurczyck, "HgCdTe Photoconductive Detectors", in Semiconductors and Semimetals, R.K. Will ardson and A.C. Beer, eds., Academic Press, New York, 18, 201 (1981).

8. G.F. Stillman, C.M. Wolfe and J.0. Dimock, "Far-Infrared

Photoconductivity in High Purity GaAs", in Semiconductors and Semimetals, R.K. Willardson and A.C. Beer, eds., Academic Press, New York, I2, 291 (1977).

9. P.R. Bratt, "Inpurity Germanium and Silicon Infrared Detectors", in Semiconductors and Semimitals, R.K. Willards on and A.C. Beer, eds., Academic Press, New York, 12 , 39-142 (1977).

10. N.M. Haegel, "Transient and Temperature Dependent Phenomena in Ge:Be and Ge:Zn Far Infrared Photoconductors", Ph.D. thesis, University of California at Berkeley, Nov. 1985; Lawrence Berkeley Laboratory publication no. 20627 (1985).

11. N.M. Haegel, "Performance and Materials Aspects of Ge:Be and Ge:Ga Photoconductors for Far Infrared Detection", M.S. thesis, University of California at Berkeley, Lawrence Berkeley Laboratory Publication No. 16694, Berkeley, California (1983).

12. J.Q. Wang, P.L. Richards, J.W. Beeman and E.E. Haller, "A stressed priotoconductive detector for far-infrared space applications", submitted to Applied Optics, Lawrence Berkeley Laboratory Publication No. 23222 (1987).

13. M.G. Stapelbroek, M.O. Petroff, J.J. Speer, D.D. Arington and C. Sayre, "Performance and Charcteristics of BIB Infrared Detectors and Detector Arrays", IRIS Specialty Group in IR Detectors, Boulder, C0, August 4, 1983. 
14. M.G. Stapelbroek, M.D. Petroff and J.J. Speer, "Attributes of BIb Detectors in Clear and Garma Radiation Enyironments", gth DARPA Strategic Space Symposium, Monterey CA, October 3-6, 1983.

15. M.D. Petroff and M.G. Stapelbroek, "Responsivity and Noise Models of Blocked Impurity Band Detectors", IRIS Specialty Group on IR Detectors, Seattle, HA, August 15, 1984.

16. M.G. Stapelbroek, M.D. Petroff, J.J. Speer and R. Bharat, "Urigin of Excess Low-Frequency Noise at Intermediate Infrared Backgrounds in BIB Detectors", IRIS Specialty Group on IR Detectors, Seattle, WA, August I5, 1984 .

17. M.D. Petroff and M.G. Stapeibroek, "Spectral Response, Gain, and Noise Models for IBC Detectors", IRIS Specialty Group on IR Detectors, August 1985.

18. E.E. Haller, private communication.

19. M.F. Mott and H.D. Twose, Advances in Physics, 10, 111 (1971).

20. B.I. Shklovskif and A.L. Efros, Electronic Properties of Doped Semiconductors, Springer-Verlag, New York (1984).

21. E.E. Haller, Infrared Phys., 25 (1/2) 257 (1985).

22. R.J. Keyes and T.M. Quist, "Low level coherent and incoherent detection in the infrared", in Semiconductors and Semimetals, R.K. Willardson and A.C. Beer, eds., Academic Press, New York, $\underline{5}, 355$ (1970).

23. M.D. Petroff and M.G. Stapelbroek, "Blocked impurity band detectors", U.S. Patent No. 4,568,960 filed 23 October 1980 , granted \& February (1986).

24. M.D. Petroff and M.G. Stapelbroek, "Blocked Impurity Band Detectors", Conf. on Extrinsic Silicon Detector Behavior, NOSC San Diego, CA April (1980)

25. M.D. Petroff and M.G. Stapelbroek, "Blocked Impurity Band Detectors: Radiation Hard High Performance LWIR Detectors", IRIS Specialty Group on IR Detectors, Menio Park, CA July (1980).

26. W.A. Kleinhans, M.O. Petroff and M.G. Stapelbroek, "Hybrid Si:As BIB detector arrays", Proceedings of the IRIS Specialty Group on IR Detectors, August 1984.

27. S.B. Stetson, O.B. Reynolds, M.G. Stapelbroek and R.L. Stermer, "Design and Performance of BIB Detector Focal Plane Arrays", Proc. SPIE 686,48 (1986)

28. F. Szmuiowicz and F. Madarz, J. App \}. Phys. 62 (6) 2533 (1987).

29. V. Hadek, J. Farhoomand, C.A. Beichman, O.M. Wats on and M.D. Jack, Appl. Phys. Lett. 46 (4) 403 (1985). 
30. V. Hadek, J. Appl. Phys. 61 (2) 773 (1987).

31. O.M. Watson and J.E. Huffman, "Germanium blockeo-impurity bano far infrared detectors", submitted to Appl. Phys. Lett. (1987).

32. A. Sherman, Chemical Vapor Deposition for Microelectronics, Noyes Publications, Park Ridge, New Jersey (1987).

33. J.W. Matthew, ed., Epitaxial Growth, Parts A and B, Academic Press, New York (1975).

34. W.A. Bryant, J. Mater. Sci. 12 (7) 1285 (1977).

35. A.T. Kuiper, C.J. van den Brekel, J. de Groot and G.W. Vel tkamp, J. Electrochem. Soc., 129 (10) 2288 (1982).

36. O.V. Tsu and G. Lucovsky, J. Vac. Sci. Tech. A, 4 (3) 480 (1986).

37. Y. Ohmachi, T. Nishiokaand and Y. Shinoda, J. Appl. Phys. 54 (9) 5466 (1983).

38. G. Bajor, K. Cadien, M. Ray and J. Greene, App1. Phys. Lett. 40 (8) $696(1982)$.

39. M. Barkai and E. Grunbaum, Thin Sol. Films, 78, 343 (1980).

40. K. Shoji, M. Hyodo, H. Veba and C. Tatsuyama, Jap. J. Appl. Phys. 22 (10) 1482 (1983).

41. G. Dubey, Thin Sol, Films, 24, 71 (1974).

42. B. Sloope and C. Tiller, J. Appl. Phys. 36 (10) 3174 (1965).

43. B. Sloope and C. Tiller, J. Appl. Phys. 38 (1) 140 (1967).

44. R. Lomas, M. Hampshire, R. Tomlinson and K. Knott, Phys. Stat. Solidi (a), 16, 385 (1973).

45. I. Khan, J. Appl. Phys, 44 (1) 14 (1973).

46. T. Asano and H. Ishiwara, Jap. J. App 1. Phys., 21 (10) L630 (1982).

47. A. Tikhonova, Sov. Phys. Crystallog., 16 (2) 345 (1971).

48. V. Vasil'ev and A. Tikhonova, Sov. Phys. Crystallog., 11 (4) 575 (1967).

49. V. Ivanov, Sov. Phys. Crystallog., 17 (5) 968 (1973).

50. L. Lavrent'eva, I. Zakharov and Y. Rumyantsev, Sov. Phys. Crystallog., 15 (4) $744(1971)$.

51. M. Berkenblit, A. Reisman and T. Light, J. Electrochem. Scc., 115

(9) $966(1968)$. 
52. A. Vervyka, E. Zhemchuzhina, A Kuznets ov and $D$. Levinson, Izvestiya Akademi i Nauk SSSR, Seriya Fizika, 37 (11) 2310 (1973).

53. G. Gavrilov and V. Evdokimov, Inorg. Materials, 13 (4) 485 (1977).

54. S. Papazian and A. Reisman, J. Electrochem. Soc. 115 (9) 961 (1968). 55. H. krautle, P. Roentgen and H. Beneking, J. Cryst. Growth, 65,439 (1983).

56. E. Roth, H. Gossenberger and J. Amick, RCA Review, December, 504 (1963).

57. D. Dumin, J. Electrochem. Soc., 117 (1) 95 (1970).

58. T. Kuech, M. Maenpaa and S. Lau, Appl. Phys. Lett., 39 (3) 245 (1981).

59. M. Maenpaa, T. Kuech, M. Nicolet, S. Lau and D. Sadana, J. Appl. Phys., s3 (2) 1076 (1982).

60. K. Zanio, L. Frads and $f$. Krajenbrink, J. Vac. Sci. Tech., 15 (1) 119 (1978).

61. V. Anikin, I. Skvortsov, I. Lapidus and L. Kolokolov, Inorg. Mater., 13 (7) 950 (1977).

62. R. Outlaw, P. Hopson, G. Halker, R, Crouch and W. Jesser, J. Vac. Sci. Tech. A, $\underline{3}$ (3) 692 (1985).

63. A. Stewart, D. Jones and G. Willeke, Phil. Mag. B, 48 (4) 333 (1983).

64. A. Immorlica, Jr., and B. Ludington, J. Cryst. Growth, 51, 131 (1981).

65. H. Nelson, RCA Review, December, 603 (1963).

66. V. Mokritskif, E. Kuritsin, B. Danilichenko and V. Shakhovtsov, Phys. Stat Solidi (a), 르, K121 (1982).

67. A. Laugier, M. Gavand and G. Mesnard, Solid State Electronics, Pergamon Press, Great Britain, 10, 77 (1967).

68. V. Marrello, J. Caywood, J. Mayer and M. Nicolet, Phys. Stat. Solidi (a), 13, $531(1972)$.

69. C. Canali, J. Mayer, G. Ottaviani, D. Sigurd and W.van der Weg, Appl. Phys. Lett., 25 (1) 3 (1974).

70. F. Ishizuka, K. Sugioka and T. Itch, J. Appl. Phys. 59 (2) 495 (1986).

71. M. Hansen, ed., Constitution of Binary Alloys, First Edition, McGraw-Hill Book Co., Inc., New York, New York (1958). 
72. F.A. Trumbore, Bell Syst. Tech. J., 39, 205 (1960).

73. A. Chernov, J. Cryst. Growth, 42, 55 (1977).

74. F.C. Eversteyn, P.J. Severin, C. H. van den Brekel and H.L. Peek, J. Electrochem. Soc., 117 (7) 925 (1970).

75. M.L. Hitchman, J. Cryst. Growth, 48, 394 (1980).

76. W.A. Claassen and J. Bloem, J. Cryst. Growth, 51, 443 (1981).

77. M.E. Coltrin, R.J. Kee and J.A. Miller, J. Electrochem. Soc., 131

(2) 425 (1984).

78. C.H. van den Brekel, Philips Res. Reports, 32, 118 (1977).

79. C.H. van den Brekel and J. 810em, Philips Res. Reports, 32, 134 (2977).

80. P.C. Rundle, J. Cryst. Growth, 11, 6 (1971).

81. A.C. Adams and J. Simpson, "Growth of Submicron Epitaxial Silicon Layers by Silane Pyrolysis", J. Electrochem. Soc., 116, 248C (1969).

82. L.N. Aleksandrov, F.L. Edelman and V.V. Voskoboinikov, "Silicon Film Growth in Vacuum by pyrolysis of silane", Vacuum, 27 (3) 145 (1977).

83. S.R. Bhola and A. Mayer, "Epitaxial deposition of silicon by thermal decomposition of silane", RCA Rev., 24, 511 (1963).

84. J. Bloem, "Effect of trace amounts of water vapor on boron doping in epitaxially grown silicon from silane", J. Electrochem. Soc., 118, 1837 (1971).

85. V.S. Chiang and D. Richman, "Growth of homoepitaxial silicon at low temperatures using silane-helium mixtures", Met. Trans. 2, 743 (1971).

86. T.L. Chu and G.A. Gruber, "Large area silicon munctions by the epitaxial growth technique (silane pyrolysis)", J. Electrochem. Soc., 114 (5) $522(1967)$.

87. M. Oruminski, "Optimization of the deposition conditions for epitaxial silicon films on Czochralski sapphire in the silane-hydrogen system", J. Electrochem, Soc., 127, 957 (1984).

88. F.L. Gittler, "Epitaxial Deposition of silicon (from silane)", J. Cryst. Growth, 17, 271 (1972).

89. D.C. Gupta, "Nonequilibrium behavior of dopants during epitaxial silicon deposition using silane", J. Electron. Mater. 1(3) 371 (1972).

90. D.C. Gupta and R. Yee, "Silicon epitaxial layers with abrupt interface impurity profiles (from low temperature pyrolysis of silane)", J. Electrochem. Soc., 116 (11) 1561 (1969). 
91. E.H. Putley, The Hall Effect and Related Phenomena, Butterworths, London (1960).

92. F.A. Trumbore, Bell Syst. Tech. J., 39, 205 (1960).

93. V.S. Ban and S.L. Gilbert, J. Crystal Growth, 31, 284 (1975).

94. C.Y. Chen, S.N. Chu and A.Y. Cho, Appl. Phys. Lett., 46 (12) 1145 (1985).

95. S. Wood, J. Greggi, Jr., and W.J. Takei, Appl. Phys. Lett., 46 (4) $371(1985)$.

96. 8.S. Meyersan, E. Ganin, D.A. Smith and T.N. Nguyen, "Low temperature silicon epitaxy by hot wall ultrahigh vacuum low pressure CVD techniques: Surface optimization", J. Electrochem. Soc., 133 (6) 1232 (1986).

97. M.J. Duchemin, M.M. Bonnet and M.F. Koelsch, "Kinetics of silicon growth under low hydrogen pressure", J. Electrochem. Soc., 125 (4) 637 (1978).

98. W.R. Burger and R. Reif, "Electrical quality of low temperature epitaxial silicon: The effect of deposition temperaturen, J. Appl. Fhys., 63 (2) 368 (1988).

99. W.L. Eisenman, J.D. Merriam and R.F. Potter, "Operational characteristics of infrared photodetectors", in Semiconductors and Semimeta 15 , R.K. Willards on and A.C. Beer, eds., Academic Press, New York, 12, 1 (1977).

100. These filters were fabricated and characterized by Gordon Stacey, Physics

Department, University of California at Berkeley (1987).

101. J.W. Beeman, Lawrence Berkeley Laboratory, private comunication. 102. V. Hadek, O.M. Watson, C.A. Beichman and M.D. Jack, Phys. Rev. B, 31 (6) 3630 (1985).

103. J.Q. Wang, P.L. Rtchards, J.W. Beeman, N.M. Haegel and E.E. Haller, Applied Optics, 25, 4127 (1986).

104. R.A. Smith, Semiconductors, second edition, Cambridge University Press, London, p.96 (1978).

105. Professor 5. Wang, University of California at Berkeley, class notes eq. 7.4 (1985). 
APPENOIX 1. FERMI LEVELS AND BUILT-IN VOLTAGE

To calculate the built-in voltage at the epilayer/substrate interface, " one needs to calculate the oifference between the Fermi levels in the pure epilayer and in the heavily-doped substrate at the temperature of interest.

The Fermi level in the heavily-doped substrate, assuming there are a neglible number of residual compensating donors relative to the number of acceptors, at low temperatures, is given by (104):

$$
E_{f}=1 / 2 E_{a}+1 / 2 k T \ln \left(N_{a} / N_{v}\right)
$$

where $E_{f}$ is the Fermi level, $E_{d}$ is the ionization energy of the acceptor impurity in the bandgap of the semiconductor, $k$ is Boltzman's constant, $T$ is the temperature, $N_{a}$ is the concentration of acceptors and $N_{v}$ is the density of states of the valence band at the temperature of interest.

To calculate the built-in voltage for the detectors made from substrates with Ga concentrations of $5 \times 10^{15} \mathrm{~cm}^{-3}$ and $2 \times 10^{16} \mathrm{~cm}^{-3}$, the following values are used:

$E_{a}=0.011 \mathrm{eV}$ for $\mathrm{Ga}$ in $\mathrm{Ge}$ at a doping level of $5 \times 10^{15} \mathrm{~cm}^{-3}$, and $0.007 \mathrm{eV}$ for $\mathrm{Ga}$ in Ge at a doping level of $2 \times 10^{16} \mathrm{~cm}^{-3}$ $k=8.65 \times 10^{-5} \mathrm{eVk}^{-1}$

$T=2.3 \mathrm{~K}$ for the lightly voped detector and $1.4 \mathrm{~K}$ for the heavily doped detector.

$$
N_{a}=5 \times 10^{15} \mathrm{~cm}^{-3} \text { or } 2 \times 10^{16} \mathrm{~cm}^{-3}
$$




$$
\begin{aligned}
& N_{v}=2.5 \times 10^{19}\left(\left(m_{h}^{*} / m_{0}\right)(T / 300)\right)^{3 / 2}\left(\mathrm{~cm}^{-3}\right) \\
& \text { where } m_{h} * / m_{0} \text { is the effective hole mass in Ge, } 0.33, \\
& N_{v}=3.18 \times 10^{15} \mathrm{~cm}^{-3} \text { at } 2.3 \mathrm{~K} \\
& N_{v}=1.5 \times 10^{15} \mathrm{~cm}^{-3} \text { at } 1.4 \mathrm{~K}
\end{aligned}
$$

The Fermi level in the $5 \times 10^{15} \mathrm{~cm}^{-3}$ substrate is then $0.0056 \mathrm{eV}$ above the valence band and the fermi level in the $2 \times 10^{16} \mathrm{~cm}^{-3}$ substrate is $0.0042 \mathrm{ev}$.

To calculate the fermi level at $2.3 \mathrm{~K}$ in the pure epilayer, the above values were used, with the exception of $E_{a}=0.044 \mathrm{eV}$ for $\mathrm{Cu}$ in Ge and $N_{a}=2 \times 10^{13} \mathrm{~cm}^{-3}$. The Fermi level is then $0.022 \mathrm{eV}$ above the valence band. The built-in voltage at the epilayer/substrate interface is the difference between the fermi levels in the respective materials and is $0.0164 \mathrm{eV}$ for the detector made from the $5 \times 10^{15} \mathrm{~cm}^{-3} \mathrm{Ga}$-doped material at $2.3 \mathrm{~K}$, and is $0.0184 \mathrm{eV}$ for the detector made from the $2 \times 10^{16} \mathrm{~cm}^{-3} \mathrm{Ga-doped} \mathrm{material} \mathrm{at} 1.4 \mathrm{~K}$.

The calculated Fermi level in the doped substrate materials can be verified by experimental dark current data. The dark curront in the detectors, at a given temperature, is a function of the number of thermally ionized holes, assuming the dark current from other sources is neglible. At $2.3 \mathrm{~K}$, detector $13-2$ made from the $5 \times 10^{15} \mathrm{~cm}^{-3}$ material had a dark current of $2 \times 10^{-13} \mathrm{~A}$ at $30 \mathrm{mV}$ applied bias and the field in the epilayer was $4.6 \mathrm{Vcm}^{-1}$. For a detector with an area of $2.25 \mathrm{~mm}^{2}$, the hole density is calculated from the current density (105):

$$
J_{p}=h u_{p} E p
$$


where $J_{p}$ is the current density in $\mathrm{Acm}^{-2}, h$ is the charge of a hole, $E$ is the electic field (taken as the maximum value which occurs at the epilayer/substrate interface), and $p$ is the hole density. This equation assumes that the current density due to thermal diffusion of carriers is negligible at the low temperatures considered here and hence this contribution to the current density is omitted. Using the following values,

$$
\begin{aligned}
& E= 4.6 \mathrm{Vcm}^{-1} \\
& u_{p}=10^{4} \mathrm{~cm}^{2} v^{-1} \mathrm{~s}^{-1} \text { for both the pure Ge epilayer and the } \\
& \quad 009 \% \mathrm{Ge} \text { IR-active layers, Dased on Hall effect measurements. } \\
& h=1.6 \times 10^{-19} \text { Coulonbs } \\
& J_{p}=\left(2 \times 10^{-13} \mathrm{~A}\right) /\left(2.25 \mathrm{~mm}^{2}\right)=8.9 \mathrm{Acm}^{-2}
\end{aligned}
$$

a hole density of $1.21 \times 10^{3} \mathrm{~cm}^{-3}$ is calculated at $2.3 \mathrm{~K}$. Now the Fermi level, $E_{f}$, can be calculated fron the hole density (101):

$$
E_{f}=\left(\ln \left(p / N_{v}\right)\right)(k T)
$$

Using the values for the variables as given above for the $5 \times 10^{15} \mathrm{~cm}^{-3}$ material at $2.3 K_{\theta} E_{f}=0.0057 \mathrm{eV}$. This is very close to the Fermi energy of 0.0056 calculated from equation 1.1 . If this procedure is repeated for the $2 \times 10^{16} \mathrm{~cm}^{-3} \mathrm{Ge}: G a$ material, using values of dark current at $1.4 \mathrm{~K}$ of $5 \times 10^{-12} \mathrm{~A}$ at $20 \mathrm{mV}$ and a detector area of $2.25 \mathrm{~mm}^{2}$, then the Fermi level is 0.0029 which is aiso very close to the value calculated from equation 1.1. 
APPENDIX II. ELECTRIC FIELD IN THE DETECTOR

The electric field in the depletion region and epilayer of a reverse biased BIB detector is calculated as follows:

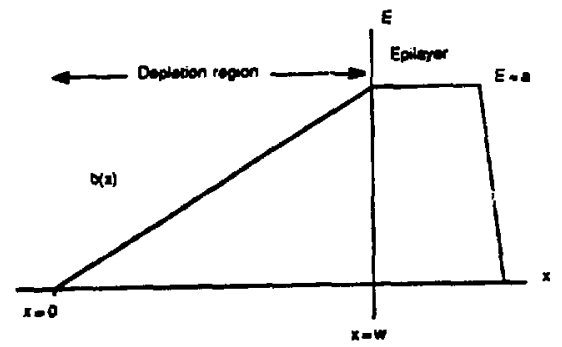

XBL BOC 1168

$$
a t+\int_{0}^{\omega} b x(d x)=\left(v_{a}-v_{b i}\right)
$$

where $a$ is the electric field in the epilayer in $\mathrm{Vcm}^{-1}$ and it is also the maximum field in the depletion region, $t$ is the epilayer thickness, $b x$ is the electric field in the depletion region at a distance $x(\mathrm{~cm})$ from the beginning of the depletion region, $V_{a}(V)$ is the applied voltage, $V_{b j}$ is the built-in voltage, and $w(\mathrm{~cm})$ is the depletion width at a given bias. In addition:

$$
\begin{aligned}
& b w=a, \text { when } x=w \\
& a t+\int_{0}^{w}(a / w) x(d x)=\left(v_{a}-v_{b i}\right)
\end{aligned}
$$


For the $5 \times 10^{15} \mathrm{~cm}^{-3} \mathrm{Ge}: \mathrm{Ga}$ detector, $t=10 \mu \mathrm{m}$, and for the $2 \times 10^{16} \mathrm{~cm}^{-3}$ detector $\mathrm{t}=7 \mu \mathrm{m}$. Table IV lists the electric field in the epilayer and depletion region for these two detectors as a function of depletion width at given biases as calculated fron the above equations. 
APPENDIX III. DARK CURRENT AS A FUNCTION OF TEMPERATURE

To determine approximately what temperature, $T$, is required for the BIB detectors to exhibit a dark current of $10^{-16} \mathrm{~A}$, the hale densities and Fermi levels derived in Appendices I and II are used in the following equation (104):

$$
T=\left(-E_{f}\right) /\left(k \ln \left(p / N_{v}\right)\right.
$$

Using equation I.2, the detector made from the $5 \times 10^{15} \mathrm{~cm}^{-3} \mathrm{Ge:Ga}$ material will have $0.6 \mathrm{~cm}^{-3}$ holes corresponding to a $10^{-16} \mathrm{~A}$ dark current. Using this value for $p$ and the previously calculated value for $E_{f}$, a temperature of $1.8 \mathrm{~K}$ is required for the dark current of $13-2$ to be reduced to $10^{-16} \mathrm{~A}$.

If the same calculations are applied to detector 16-8, made from the $2 \times 10^{16} \mathrm{~cm}^{-3} \mathrm{Ge}: \mathrm{Ga}$ material, using a hole density of $1.26 \mathrm{~cm}^{-3}$ corresponding to a dark current of $10^{-16} \mathrm{~A}$, then a temperature of $1 \mathrm{~K}$ is required to reduce the dark current to $10^{-16} \mathrm{~A}$. 


\section{APPENDIX IV. DENSITY OF STATES FUNCTION}

To determine whether the heavily-doped material will freeze out completely with decreasing temperature, one must know the density of states as a function of energy between the top of the band edge and the bottom of the impurity band. For nondegenerately doped material, with a very low compensation level, Shklovskii and Efros (20) predict that the denstty of states function is Gaussian, centered about the average energy of the impurity:

$$
N(E)=\left(N_{a}\right) /\left(r-\exp \left(-E^{2} / r^{2}\right)\right.
$$

where $N(E)$ is the density of states as a function of energy, $N_{a}$ is the concentration of acceptors, $Y$ is a parameter describing the energy spread of the impurity band and is the half-width at half-maximu of the Gaussian function, and $E$ is the difference between the average energy of the impurity and the energy above the valence band edge. The paraneter $y$ is defined as:

$$
Y=0.266 E_{a}\left(H_{a} / H_{d}\right)^{1 / 4}
$$

where $E_{d}$ is the average ionization energy of the impurity and $H_{d}$ is the compensation level. This equation assumes $Y \ll E_{f}$ and $N_{d} / N_{a} \ll 1$. In addition, the Fermi level at low temperatures and Iow compensation is approximated as:

$$
E_{f}=0.61 E_{a}
$$


123

These equations yield values of $Y=0.0001 \mathrm{eV}$ for the $5 \times 10^{15} \mathrm{~cm}^{-3}$ Ge:Ga material with $N_{d}=10^{10} \mathrm{~cm}^{-3}$, and $y=0.0002 \mathrm{eV}$ for the $2 \times 10^{16} \mathrm{~cm}^{-3} \mathrm{Ge}: G a$ material with $N_{d}=5 \times 10^{11} \mathrm{~cm}^{-3}$. The Fermi energies are slightly higher than those predicted in Appendix I: $0.0067 \mathrm{eV}$ and $0.0043 \mathrm{eV}$ for the $5 \times 10^{15} \mathrm{~cm}^{-3}$ and $2 \times 10^{16} \mathrm{~cm}^{-3}$ materials, respectively. This model predicts that the spread in energy of the impurity band is very small and that the density of states drops to a negligible amount within $0.001 \mathrm{eV}$ of the average energy of the inpurity band. It is questionable whether this model accurately predicts the density of states for these heavily doped materials, and the true test will come when the dark current for BIB detectors made from these materials is measured at low temperatures. 